\title{
Cognitive Radio Transmitter With a Broadband Clean Frequency Spectrum
}




\section{COGNITIVE RADIO TRANSMITTER WITH A BROADBAND CLEAN FREQUENCY SPECTRUM}

Saqib Subhan 


\title{
The Graduation Committee:
}

Chairman:

Secretary:

Prof.dr.ir. P.G.M. Apers University of Twente

Promotor:

Prof.dr.ir. P.G.M. Apers University of Twente

Prof.dr.ir. B.Nauta University of Twente

Assistant Promotor:

Members:

Dr.ing. E.A.M. Klumperink University of Twente

Prof.dr.ir. P.G.M. Baltus Eindhoven University of Technology

Prof.dr. R.B. Staszewski Delft University of Technology

Prof.dr.ing. P.J.M. Havinga University of Twente

Prof.dr.ir. F.E. van Vliet TNO/ University of Twente

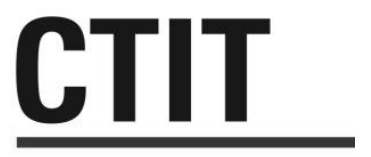

CTIT Ph.D. Thesis Series No. 14-310.

Center for Telematics and Information Technology.

P.O. Box 217, 7500 AE

Enschede, The Netherlands.

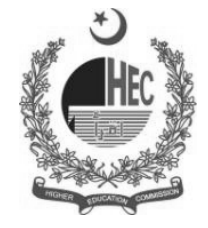

This research is supported by the Higher Education Commission

(HEC), Government of Pakistan.

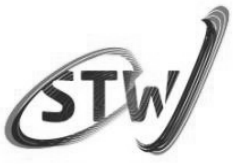

Enabling new technology

\begin{abstract}
This research is partly supported by the Dutch Technology Foundation (STW), which is part of the Netherlands Organization for Scientific Research (NWO) and partly funded by the Ministry of Economic Affairs, Agriculture and Innovation (08081 ADREM Radio) .
\end{abstract}

Title: Cognitive Radio Transmitter with a Broadband Clean Frequency Spectrum

ISBN: 978-90-365-3677-6.

ISSN: $1381-3617$.

DOI: $10.3990 / 1.9789036536776$.

Copyright (C) 2014 Saqib Subhan, Enschede, The Netherlands. 


\title{
COGNITIVE RADIO TRANSMITTER WITH A BROADBAND CLEAN FREQUENCY SPECTRUM
}

\author{
DISSERTATION \\ to obtain \\ the degree of doctor at the University of Twente, \\ on the authority of the rector magnificus, \\ prof. dr. H. Brinksma, \\ on account of the decision of the graduation committee, \\ to be publicly defended \\ on Wednesday the $2^{\text {nd }}$ of July 2014 at 16:45
}

by

Saqib Subhan

born on $2^{\text {nd }}$ June 1981

in Lahore, Pakistan 
This dissertation has been approved by

The Promotor:

Assistant Promotor:
Prof. dr. Ir. Bram Nauta

Dr.ing. Eric. A.M. Klumperink 


\section{ABSTRACT}

The tremendous increase in wireless communication over the last few decades has led to a congestion of the radio frequency (RF) spectrum, which is utilized for transmission and reception of information. As suitable RF spectrum is scarce, attempts are being made to use the RF spectrum in a more intelligent efficient way. A Cognitive Radio addresses this problem by Dynamic Spectrum Access, i.e. measure which spectrum is temporarily locally free and then use it. The Cognitive Radio transmitter needs to be flexible to be able to transmit where ever there is free spectrum available.

Conventional transmitters not only produce the desired upconverted information signal but also many unwanted harmonics of the local oscillator (LO) and distortion products related to the baseband signal. These unwanted products have been usually suppressed using dedicated RF filters which are narrowband and are not flexible. For Cognitive Radio transmitters flexibility is a key requirement, and hence other techniques are wanted to suppress unwanted products, without using the inflexible filters. Moreover, agile operation of the cognitive radio transmitter in a broad band is wanted.

Previous research has shown that polyphase multipath circuits can in principle cancel a large number of harmonics and distortion products. However, a solution for wideband polyphase baseband signal generation including digital-to-analog conversion and filtering was lacking. Moreover, the upconversion was done using a large number of paths which takes quite some chip area and is not very power efficient. In this work a less complex and more power efficient implementation of this technique is proposed. The proposal is actually based on a combination of three techniques, namely: 1) 8-path polyphase upconversion, 2) tuning of the LO duty cycle ratio to close to $7 / 16$ and 3) a tunable first order RF filter. The combination of these three techniques allows to suppress all unwanted products to more than $40 \mathrm{~dB}$ below the desired signal. It is possible to improve this further if a tunable RLC network with high quality factor is used at the RF output.

The multiphase baseband signals required for an 8-path upconversion can be generated using a simplified vector modulator type of architecture. In order to allow for a Spurious Free Dynamic Range of $50 \mathrm{~dB}$, the use of a Digital to Analog Converter (DAC) with a resolution of at least 7 bits is proposed. It is shown that it is possible to cancel the first dominant DAC image by using a polyphase DAC architecture, which relaxes analog reconstruction filtering requirements.

To verify the functionality of the proposed techniques, a flexible 8-path transmitter chip was fabricated in a 160 nanometer CMOS technology. The transmitter works over 3 octaves in frequency from $100 \mathrm{MHz}$ to $800 \mathrm{MHz}$. Measurements show that the frequency 
agile transmitter achieves a broadband clean output spectrum where all unwanted products are at least $40 \mathrm{~dB}$ below the wanted transmit signal. This is the first polyphase multi-path transmitter combining the baseband multi-phase generation and RF circuit on one chip. Compared to other harmonic rejection transmitter designs with similar frequency range, it is more power efficient and has better LO leakage and image rejection. Note that this chip suppresses ALL LO harmonics and distortion products for ALL frequencies, without any external filters. 


\section{SAMENVATTING}

De enorme toename van draadloze communicatie in de afgelopen decennia begint te leiden tot verstopping van het radio frequentie (RF) spectrum dat wordt gebruikt voor het verzenden en ontvangen van informatie. Omdat geschikt RF spectrum schaars is, probeert men het spectrum op een meer intelligente efficiente wijze te gebruiken. Een Cognitieve Radio doet dit via dynamische spectrum toegang door te meten waar spectrum (tijdelijk plaatselijk) vrij is en dan te benutten. Een Cognitieve Radio zender moet daarvoor flexibel zijn qua zendfrequentie.

Zenders dienen idealiter alleen het basisband informatie signaal op te converteren naar de zendfrequentie. Daarbij ontstaan echter ook vele ongewenste nevenproducten, ondermeer harmonischen van de voor het converteren gebruikte lokale oscillator (LO) en vervormingsproducten van het basisband signaal. Deze ongewenste nevenproducten worden in een conventionele zender meestal onderdrukt met behulp van speciale RF-filters die smalbandig zijn en niet felxibel verstembaar. Voor een cognitieve radio zender is flexibiliteit een eerste vereiste, zodat vaste filters onpraktisch zijn. Darom zijn er nieuwe technieken nodig, die een flexibele zendfrequentie mogelijk maken zonder het radio spectrum onnodig te vervuilen.

In voorgaand onderzoek is aangetoond dat polyfase multi-pad circuits in principe in staat zijn een groot aantal harmonischen en distorsieproducten te onderdrukken. Een oplossing voor de breedbandige polyfase basisband signaalgeneratie ontbrak echter. Ook was een erg groot aantal signaalpaden nodig, wat nadelig is voor chipoppervlakte en energie gebruik. In dit onderzoek wordt een meer compacte en energie-efficiënte uitvoering van deze techniek voorgesteld op basis van een combinatie van drie technieken: 1 ) 8-pad polyfase frequentie conversie; 2) afregeling van de duty cycle van het LO signaal op ongeveer $7 / 16$; 3) toepassing van een eerste order laagdoorlaat RF filter met regelbare capaciteit. De combinatie van deze drie technieken kan alle LO harmonischen en distorsie producten tenminste $40 \mathrm{~dB}$ onderdrukken ten opzichte van het gewenste zendsignaal. Het is mogelijk dit verder te verbeteren als een afstembaar R-L-C netwerk met hoge kwaliteitsfactor gebruikt wordt op de uitgang. Het benodigde polyfase basisband signaal kan worden gegenereerd met behulp van een vereenvoudigde vectormodulator in het digitale domein. Om een "Spurious Free Dynamic Range" van meer dan 50dB mogelijk te maken, wordt voorgesteld een digitaal-analoog converter (DAC) met een resolutie van tenminste 7 bits te gebruiken. Tevens wordt aangetoond dat het mogelijk is om de eerste DAC image the onderdrukken met behulp van een meerfase DAC architectuur, waardoor minder analoge reconstructie filtering nodig is achter de DAC 
Om de werking van de voorgestelde technieken te demonstreren is een chip ontworpen en gefabriceerd in een 160 nanometer CMOS-technologie. De zender werkt over 3 octaven in frequentie; van $100 \mathrm{MHz}$ tot $800 \mathrm{MHz}$. Deze flexibele zender blijkt daarbij in staat breedbandig alle ongewenste nevenproducten meer dan $40 \mathrm{~dB}$ onder het gewenste zendsignaal te houden. Dit is de eerste chip die een polyfase multi-pad zender implementeert inclusief de meerfasige basisband signaalgeneratie. Vergeleken met andere zender chips die harmonischen onderdrukken in hetzelfde frequentiebereik, is deze chip zuiniger terwijl de LO-signaal emissie en spiegelonderdrukking ook beter is. Met name is bijzonder dat deze chip ALLE LO harmonischen en distorsie producten breedbandig onderdrukt voor ALLE frequenties, zonder gebruik te maken van externe filters. 


\section{Contents}

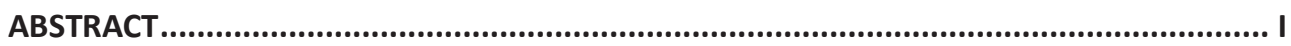

SAMENVATTING .................................................................................................. II

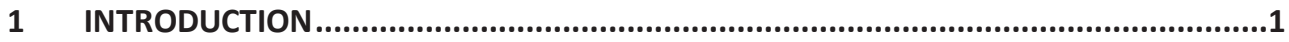

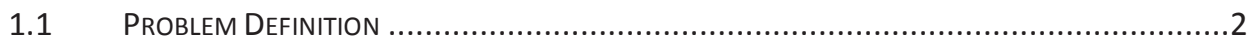

1.2 Transmitter Terminology .......................................................................... 4

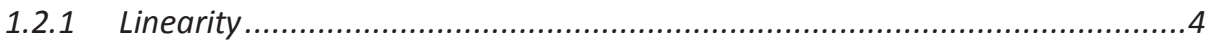

1.2.2 Error Vector Magnitude .................................................................

1.2.3 Spectral Mask...................................................................................

1.2.4 Efficiency.......................................................................................

1.2.5 LO Harmonics.............................................................................. 6

1.2.6 DAC replica images........................................................................

1.2.7 Output Noise ................................................................................... 7

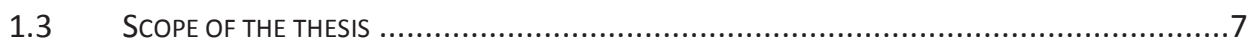

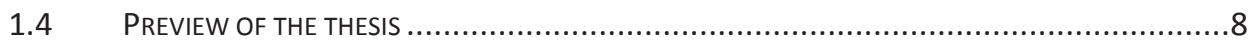

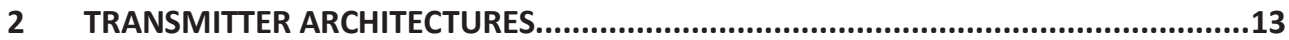

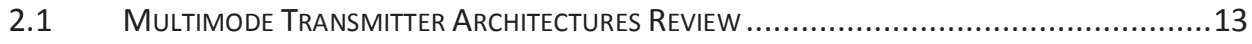

2.2 HARMONIC AND DISTORTION SUPPRESSION TECHNIQUES ..........................................16

2.2.1 Polyphase Multipath Technique ......................................................... 18

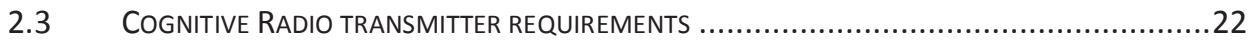

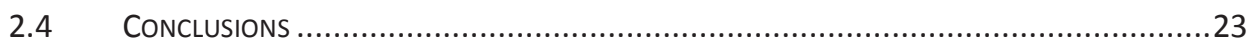

3 POLYPHASE MULTIPATH TRANSMITTER SYSTEM DESIGN ..................................29

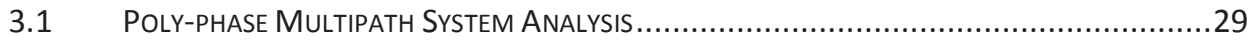

3.1.1 Multipath System with a First order RC filter at the output.........................31

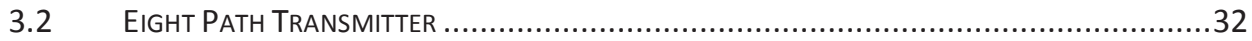

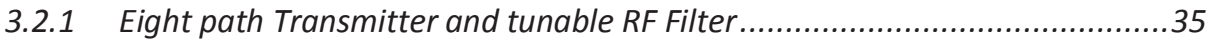

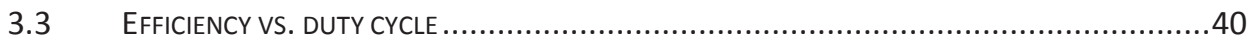

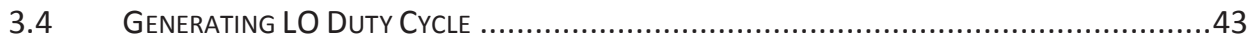

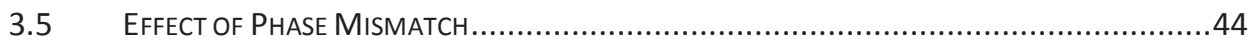

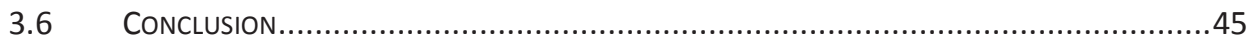

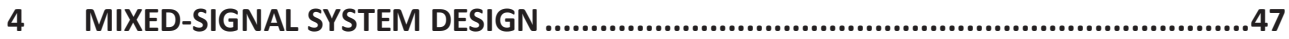

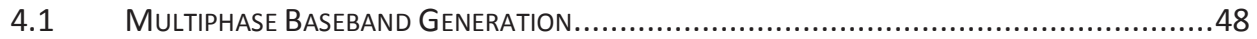

4.2 DAC Resolution Impact on A Poly-Phase Multipath Transmitter ..........................53

4.2.1 Multipath Technique applied to a Uniform DAC with ideal Phase Shifter......53 
4.2.2 Multipath Technique applied to a Uniform DAC with Mixer as Phase Shifter 57

4.3 DAC RePLICA IMAge CANCELLATION .................................................................59

4.3.1 Suppression of DAC images due to summation of parallel DAC output.........59

4.3.2 Suppression of DAC images in Polyphase Multipath Upconversion...............63



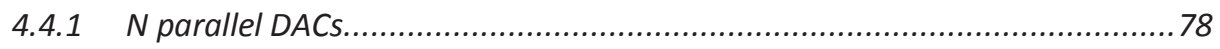

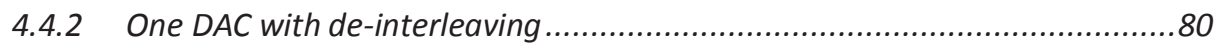

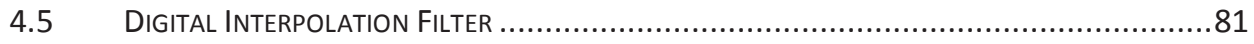

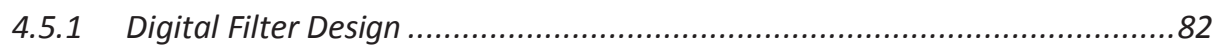

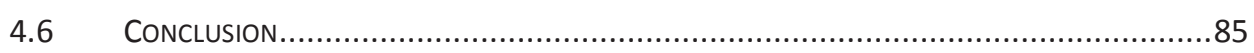

5 POLYPHASE MULTIPATH TRANSMITTER IMPLEMENTATION..................................87

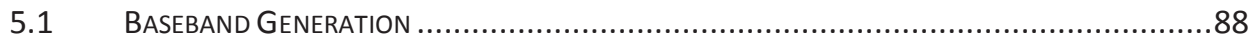

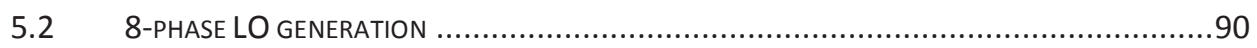

5.2.1 Generating $1 / 8$ duty cycle for sample \& hold switch ...............................91

5.3 Duty-CyCle Control Circuit Design.........................................................92

5.3.1 Duty-Cycle for the required accuracy.................................................93

5.3.2 Duty-Cycle over a Frequency Range ................................................95

5.3.3 Duty-Cycle variation with Temperature ...............................................95

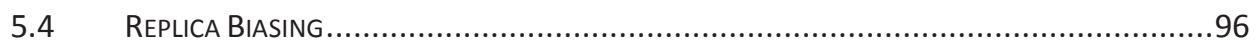

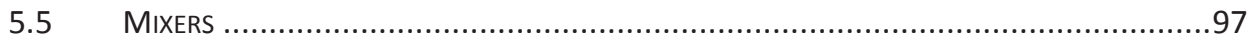

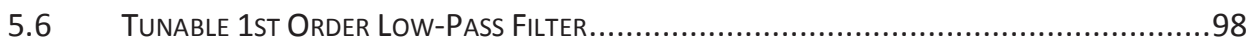

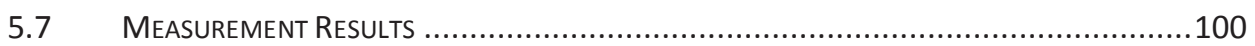

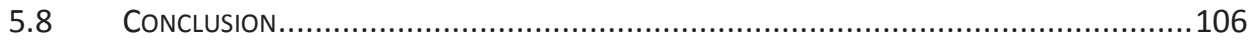

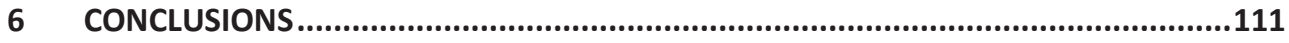

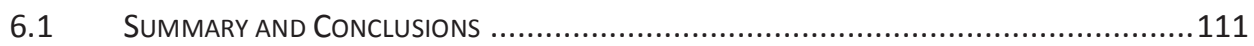

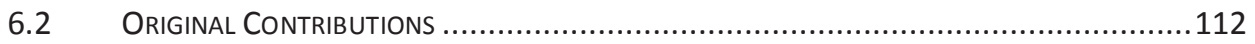

6.3 RECOMMENDATIONS FOR FUtURE WORK..................................................113

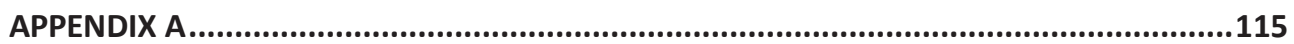

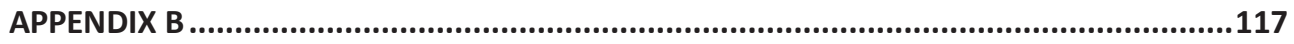

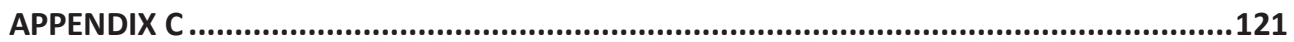

ACKNOWLEDGEMENTS ..............................................................................124

LIST OF PUBLICATIONS.................................................................................127

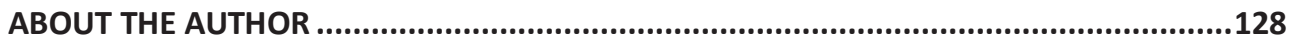




\section{CHAPTER ONE}

\section{Introduction}

The explosive growth in wireless communications has led to a plethora of wireless communication standards. Mobile handsets now commonly support multi-band GSM, WCDMA, WLAN, Bluetooth, GPS, FM radio and more. Laptops and tablets also commonly support at least several WLAN radio standards, and more functionality is continuously added. Radio standards have been proposed to serve different purposes. GSM for example is mainly developed for voice calls using the terrestrial infrastructure, Bluetooth for short distance personal area network data transfer and recent WLAN standards for fast computer network access. New extended versions of standards are regularly introduced to support new functionality or higher data rates (e.g. different WLAN version like $802.11 \mathrm{~b}, \mathrm{~g}, \mathrm{n})$. Each of the radio standards defines a communication protocol and frequency band, which was conventionally supported by a dedicated integrated circuit (IC) with external antenna and passive components to realize radio transceiver functionality (transmit and receive). As the number of these standards grows, the cost and area required to put all the ICs and associated components on one device also increases. Therefore there is a need to somehow combine functionality and re-use hardware to support different standards onto one IC. This has led to the concept of a Software Defined Radio (SDR) or its more ideal version the Software Radio [1]. A SDR contains reconfigurable radio hardware, where most of the functionality of the transceiver (transmitter and receiver) is defined in software. To reuse the same hardware frontend, it must be flexible enough to meet the requirements of all the different standards to be supported.

Each of the different protocols mentioned above uses a certain frequency band to send its data. As an example the GSM protocol uses $800 / 900 \mathrm{MHz}$ or the $1800 / 1900 \mathrm{MHz}$ frequency band. Bluetooth, WLAN among others, communicates on the $2.4 \mathrm{GHz}$ band. The frequency band a certain communication protocol uses is assigned by a regulating authority such as the Federal communication commission (FCC) in the United States or the OFCOM in the United Kingdom. Most of the terrestrial communication protocols are utilizing the frequency spectrum upto around $6 \mathrm{GHz}$. Each operator of a communication infrastructure has to buy a license from the regulator in the country where it is operating to have the right to use that particular frequency spectrum. This can be very expensive [2] as there is limited spectrum and there are quite some competitors. As shown in the FCC frequency allocation 
table $[3,4]$ most of the usable bands have already been allocated to commercial users, i.e. there seems to be a spectrum scarcity problem.

However studies [5] suggest that the spectrum is far from fully utilized for a given timeframe at a particular location. Therefore, more efficient utilization of the frequency spectrum may help to alleviate the spectrum scarcity problem. This led to the concept of cognitive radio [6]. The cognitive radio (CR) would actively monitor the frequency spectrum and look for unutilized frequency spots also known as white spaces. The cognitive radio would then dynamically alter or set its transmission parameters such as frequency, power etc, to be able to utilize those white spaces. This process is also called Dynamic Spectrum Access. The key issue here is that the incumbent users (primary users) of the frequency spectrum do not undergo any harmful interference during the whole process.

The cognitive radio can be seen as an extension of the software defined radio. Both require a flexible radio frontend to cater to different communication standards at different transmission frequencies. This thesis focuses on the transmitter part of a cognitive radio transceiver. The challenge facing such a transmitter is defined in the next section.

\subsection{Problem Definition}

The main task of a Radio Frequency (RF) transmitter is to shift a low frequency baseband signal to a higher RF signal. Moreover, the transmitter should also amplify the input signal, in order to provide the required power to the antenna load. This can be represented in the frequency spectrum as shown in Figure 1.1: two tones at the input around DC are upconverted to the output around a so-called "Local Oscillator" frequency, or simply abbreviated as "LO". Up-conversion can in theory be realized using linear but time-variant networks containing switches driven by the LO. The switching can be modeled as a multiplication with a square-wave, which will not only lead to up-conversion around LO, but also to up-conversion around higher harmonics of LO. Moreover, as the switching but also the amplification is realized exploiting nonlinear transistors, extra unwanted terms due to non-linearity are produced at the output. Clearly such terms are problematic, as they occupy extra spectrum and pose interference to other users.

A common direct conversion transmitter architecture can be realized by the blocks given in Figure 1.2. A Digital to Analog Converter (DAC) converts the digital baseband to the analog domain, while the Low Pass Filter (LPF) filter removes or suppresses the undesired frequency components coming out of the DAC. The mixer and the Power Amplifier (PA) provide the frequency translation and signal amplification 


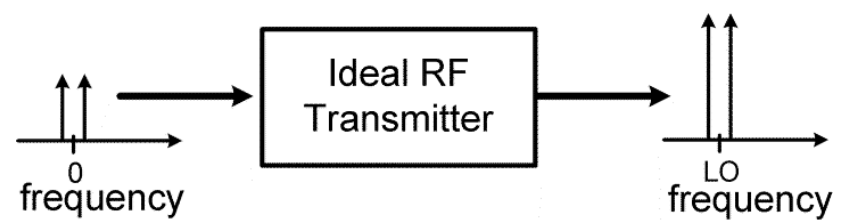

Figure 1.1. Ideal RF Transmitter Frequency Spectrum

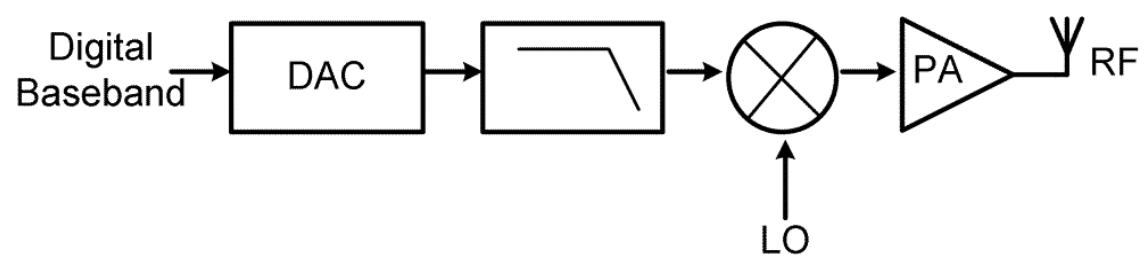

Figure 1.2. Direct Conversion Transmitter

The output spectrum of such kind of a transmitter can be similar to the one shown in Figure 1.3, which differs significantly from the output spectrum of an ideal transmitter shown in Figure 1.1. Here apart from the desired signal around the LO frequency, there are other unwanted frequency components such as IM3 ( $3^{\text {rd }}$ order inter-modulation distortion), $\mathrm{HD}_{3}$ ( $3^{\text {rd }}$ order harmonic distortion), DAC $\mathrm{img}_{\text {img }}$ (remnants of the signal image around the DAC clock which have not been completely filtered) and LO harmonics (due to time-variant behavior of switching mixers).

In order to reduce these unwanted spectral components, an RF Band Pass Filter (BPF) could be employed at the output of the transmitter as shown in Figure 1.4. This BPF can suppress most of these unwanted components, but the problem for a cognitive or software defined radio application is the desire for flexibility in frequency. Most off-chip filters are dedicated for one particular frequency band, so a multiband transmitter would require multiple of these filters. External filters would also add to the cost and size of the Integrated circuit. On chip filters generally have a limited tuning range, so they are also not very flexible. Additionally if inductors are used to implement these filters, they would also consume a lot of chip area. Since a cognitive radio transmitter has to cover a wide band, a solution is desired which can be flexible and can be integrated on chip while not requiring separate off chip filters for each RF band. 


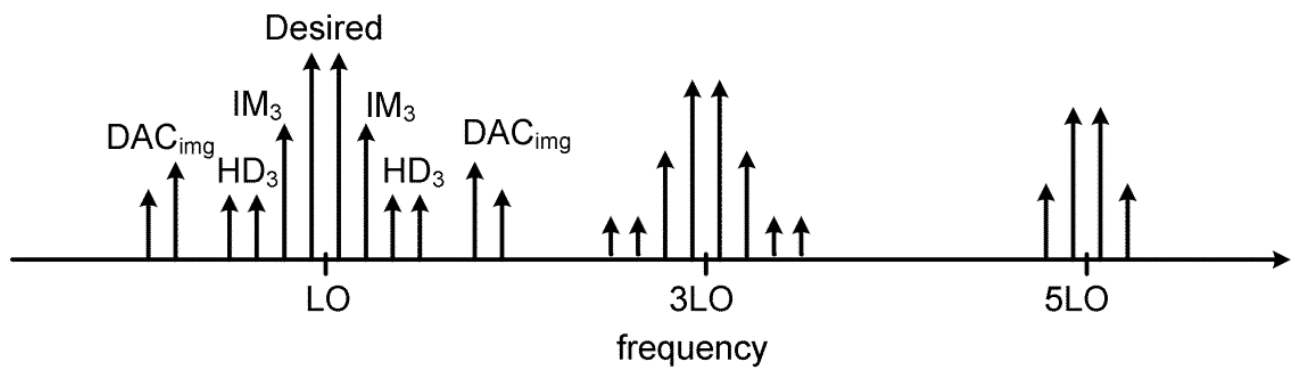

Figure 1.3 An output spectrum of the transmitter in Figure 1.2

A possible solution to overcome these challenges, could materialize in the form of switched capacitor (SC) N-path filter [7], which can achieve very high Q, while being tunable in center frequency by its clock frequency. The idea of N-path filters is quite old [8], but currently experiencing a revival [9-11]. They are flexible and easy to integrate on chip. However, handling sufficient RF power might still be challenging, moreover they still produce uncancelled harmonics. Another possible solution could be based on the use of MEMS based devices for use in tunable filters [12,13], but these suffer from limitations in tunability and linearity. Another possible solution which could be able to overcome the problems mentioned above is the use of very high frequency digital to analog converters (DACs). Recently they have become very competitive [14-16]. Although the harmonic problem would still be there, and power consumption may still be of concern.

\subsection{Transmitter Terminology}

Some key transmitter related terminologies that will be used in this thesis are introduced below, whereas their relevance for cognitive radio is also discussed briefly.

\subsubsection{Linearity}

As mentioned in section 1.1, the baseband to RF upconversion process results in nonlinearity. This results in distortion, both intermodulation distortion and harmonic distortion, which may cause interference to other users. Harmonic distortion is due to the baseband distortion combining with the LO harmonics to appear at the RF. Intermodulation distortion mainly effects nearby channels, and degrades the error vector magnitude (EVM) or bit error rate. Depending upon the input spectrum, the harmonic distortion may appear further away in the output spectrum from the desired channel, but can interfere with other users of the frequency spectrum too, especially if sufficient filtering is not present. 


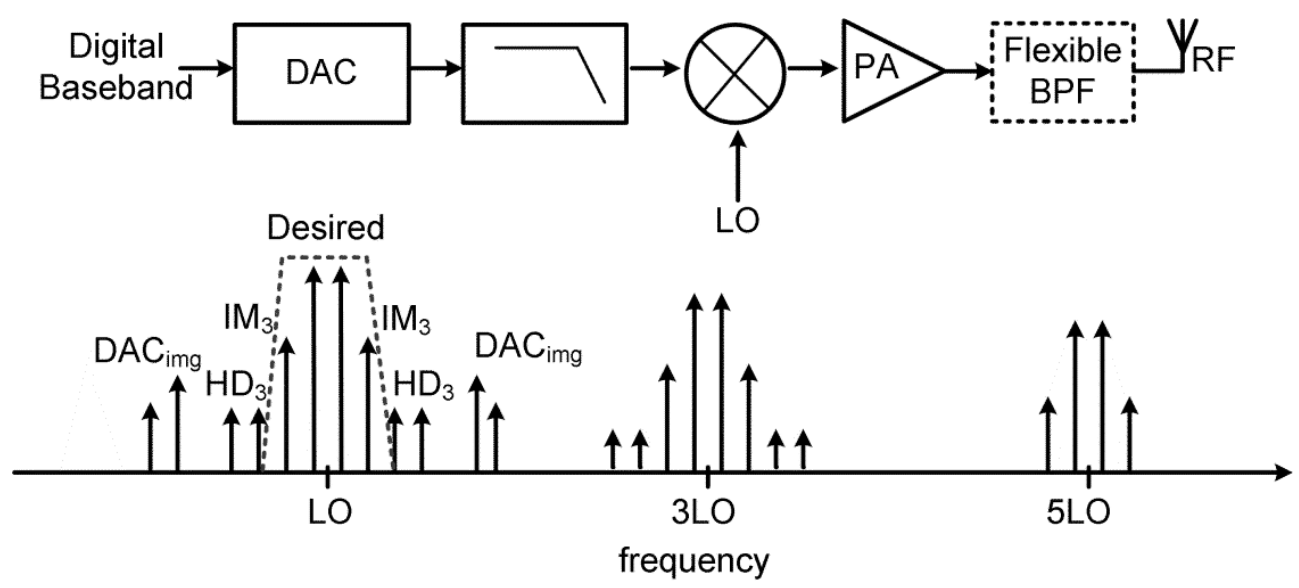

Figure 1.4 An RF BPF can suppress the unwanted spectral components.

\subsubsection{Error Vector Magnitude}

Due to several transmitter impairments, e.g. nonlinearity in the signal path, I-Q imbalance and (phase) noise, the transmitted constellation points of a digitally modulated transmit signal deviate from their ideal locations. The magnitude of the error vector between the ideal constellation point and the point to be measured, normalized by the ideal signal vector defines the error vector magnitude. This performance parameter is often used to judge the overall suitability of a transmitter to transmit high-order complex signal constellations.

\subsubsection{Spectral Mask}

The spectral mask quantifies how much power a transmitter is allowed to transmit as a function of frequency. An arbitrary spectral mask is shown in Figure 1.5, where the top of the dotted line defines the maximum allowed signal power, while the decreasing sides of the spectral mask define the maximum radiated power in neighboring and far-out bands. This mask defines the limit on the acceptable harmonics and distortion power. The spectral mask requirements for a Cognitive Radio Transmitter are discussed in Chapter 2 section 2.3 .

\subsubsection{Efficiency}

The power efficiency is a ratio between useful RF output power and the required DC power consumed to produce this power. Often, there is a trade-off between efficiency and linearity. 


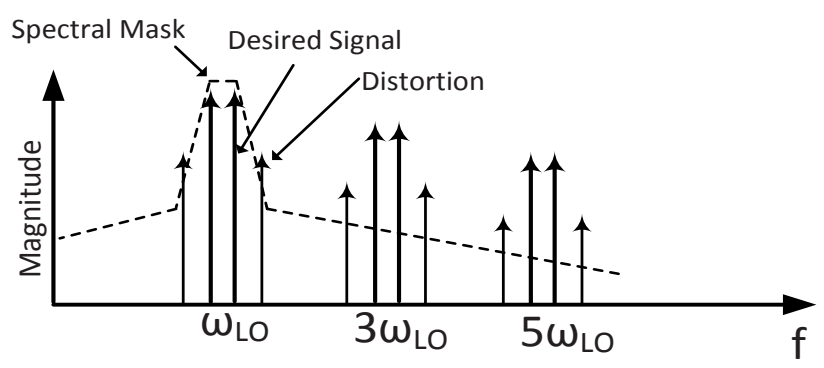

Figure 1.5. An arbitrary Spectral Mask

E.g. a transistor biased as class-A amplifier is more linear than for class-B or class-C biasing [17] but less power efficient. We will use class-A amplifiers in this thesis as linearity is important for complex modulated signals with high spectral efficiency [17].

The efficiency could be just confined to the final output amplifier. In that case it is usually defined as the drain efficiency. Here single tone output is assumed.

Drain Efficiency $=\mathrm{P}_{\mathrm{RF}} / \mathrm{P}_{\mathrm{DC}}$ amp

where $\mathrm{P}_{\mathrm{RF}}$, is the sinusoidal output power and $\mathrm{P}_{\mathrm{DC} \text {,amp }}$ is the $\mathrm{DC}$ power consumed in the output amplifier. However, there can also be significant $\mathrm{DC}$ power consumption $\left(\mathrm{P}_{\mathrm{DC}, \mathrm{BB}}\right)$ in the baseband (BB) filters and amplifiers, mixers ( $\left.\mathrm{P}_{\mathrm{DC} \text {,mixers }}\right)$ or multiphase $\mathrm{LO}$ generation $\left(\mathrm{P}_{\mathrm{DC}, L O G E N}\right)$. The total efficiency of the upconversion can then be defined as:

Total Efficiency $=\mathrm{P}_{\mathrm{RF}} /\left(\mathrm{P}_{\mathrm{DC}, \text { amp }+ \text { mixer }}+\mathrm{P}_{\mathrm{DC}, \text { LOGEN }}+\mathrm{P}_{\mathrm{DC}, \mathrm{BB}}\right)$

The power consumed in the Digital to analog converting function may also be included in the power consumption of the baseband paths, especially for completely digital solutions. An analysis on efficiency of the upconverter presented in this thesis is given in Chapter 3 section 3.3, while the measurement of the total efficiency of the implemented Cognitive Radio Transmitter is presented in Table 5.1. The output power requirements for a cognitive radio transmitter are discussed in section 2.3 .

\subsubsection{LO Harmonics}

Frequency translation in transceivers usually takes place due to mixers, which shift the frequency up (in case of transmitters) or down (in case of receivers). Most of these mixers are implemented using hard switching mixers as they provide higher conversion gain and better linearity compared to soft switching mixers. Hard switching results in harmonics of 
the LO frequency which can be dominant, if they are not appropriately suppressed. Techniques to suppress these harmonics are discussed in chapters 2 and 3.

\subsubsection{DAC replica images}

When the digital baseband is converted to the analog domain via a Digital to analog converter (DAC), replica images of the desired signal occur around the sample frequency of the DAC. If appropriate filtering is not present they can become dominant and interfere with other users of the frequency spectrum. DAC replica images can be suppressed by baseband filtering or by baseband filtering in combination with increasing the sample rate of the digital baseband via digital interpolation (see chapter 4).

\subsubsection{Output Noise}

Just like any other radiation, transmitter output noise may increase the noise floor of other radio devices and should be limited in value. The noise emanating from the transmitter can have several causes, e.g. wideband thermal noise, phase-noise of the mixer LO-signal and or up-converted thermal noise and $1 / f$ noise of various components utilized for the upconversion and amplification. Noise at the output could also be due to the quantization noise due to limited DAC resolution. In conventional transmitters the thermal noise is often dominant in the frequency spectrum far away from the desired channel, while the quantization noise, phase noise and flicker noise is more dominant close to the desired channel. The Quantization noise can be reduced by increasing the DAC resolution, or by increasing the baseband filter order.

\subsection{Scope of the thesis}

Section 1.1 presented challenges facing the design of a Cognitive Radio RF Transmitter. The state-of-the-art and previous work on flexible transmitter architectures will be discussed in chapter 2 in detail. One of the transmitter architectures discussed there is the polyphase multipath technique on which the present work will build. It will be shown that this technique can simultaneously suppress local oscillator (LO) harmonics and sideband products originating from time variant mixer behavior, as well as many distortion products due to nonlinearity. This can in principle be done without any dedicated filtering, which is a nice asset for cognitive radio. If the number of signal paths is increased, a larger number of harmonics and sidebands can be suppressed. An 18-path up-converter [18] can suppress uptill the $17^{\text {th }}$ harmonic of the LO. However the generation of 18 LO phases limits the frequency range and consumes significant power. Moreover, in [18] the multiphase 
baseband signals required to drive the multiple mixers were generated off chip. Actually, only single-tone sine wave was used, and efficient multi-phase baseband signal generation was recommended as "future work". We will motivate in chapter 3 that it makes sense to reduce the number of paths, to reduce complexity in terms of the number of baseband signals and hence the number of DACs and baseband filters. Hence, the aim will be to reduce the number of paths while maintaining or improving the harmonic suppression characteristics. It will be shown that duty cycle control of the LO can nicely complement the multipath polyphase technique if we choose an 8-path transmitter.

The main goal of this thesis is to explore design options for a flexible RF transmitter which does not require dedicated RF filters to suppress the harmonics and distortion products emanating from an upconversion process. The thesis builds upon the polyphase multipath concept, previously proposed in [19]. After a critical evaluation of several design options, a transmitter architecture is proposed that exploits LO duty-cycle control combined with an 8-path polyphase up-converter. Switched transconductor mixers [20] directly driving the antenna are used for wideband upconversion. If the antenna does not sufficiently reduce far out residual signals or noise a simple filter can be added. Depending on the requirements and frequency range, a first order low-pass filter can be added or an elementary L-C band pass filter, with variable capacitance. A variable high-frequency external clock is used for generating the multi-phase on-chip clock needed for driving the mixer switches. The frequency range targeted is the below $900 \mathrm{MHz}$ frequency bands, which are being opened up for unlicensed devices [21, 22]. To demonstrate the effectiveness of the techniques, a demonstrator IC is designed which exploits one (external) DAC to generate all the baseband phases in a time-interleaved fashion. The chip contains the de-interleaving hardware, baseband buffers with baseband filters, upconversion power-mixers and the LOgeneration hardware including the duty-cycle control. Measurements over multi octaves of frequency $(100-800 \mathrm{MHz})$ show that all unwanted products can be kept $<-40 \mathrm{dBc}$ at better power efficiency than competing designs, including [18].

\subsection{Preview of the thesis}

In chapter 2, a brief overview of some of the recent transmitter architectures which focus on flexible transmitter concepts is presented. This chapter also presents some harmonic suppression techniques used in literature. The properties of the Polyphase Multipath Technique are reviewed and it is motivated why this technique is used as a basis for the flexible transmitter concept IC design presented later in the thesis.

In Chapter 3, design options for the implementation of the Polyphase Multipath technique [18] are considered and the motivation for selecting an 8-path transmitter is presented. This 
choice is largely affected by the benefits of a particular duty cycle of the LO, which is a degree of freedom in the suppression of the harmonics. The efficiency versus duty cycle is also analyzed to show that efficiency does not degrade significantly when selecting the optimum duty cycle for harmonic suppression. Finally, LO-generation requirements and phase mismatch are analyzed.

Chapter 4 discusses the mixed signal system design, which was not previously explored. Functionally, the polyphase multipath upconversion requires polyphase baseband signals to drive the mixers. Hence the generation of digital multiphase baseband signals is explored, considering baseband DAC resolution requirements for the polyphase multipath transmitter. The baseband DAC replica image cancellation properties in a polyphase multipath upconversion system are analyzed, in an attempt to relax the baseband filtering requirements. Also some baseband DAC implementation issues are discussed along with interpolation filter requirements.

Chapter 5 presents the complete circuit design of the 8-path transmitter in a 160nm CMOS process. Measurement results of the chip demonstrate the effectiveness of the proposed circuit techniques and benchmark achieved results to that of competing design.

Chapter 6 presents the conclusions and suggestions for future work.

\section{References}

[1] J. Mitola, "The software radio architecture," Communications Magazine, IEEE, vol. 33, pp. 26-38, 1995.

[2] K. Binmore and P. Klemper, "The Biggest Auction Ever: the Sale of the British $3 G$ Telecom Licences," Economic Journal, pp. 74-95, Mar 2002.

[3] FCC. FCC ONLINE TABLE OF FREQUENCY ALLOCATIONS. Available: http://transition.fcc.gov/oet/spectrum/table/fcctable.pdf

[4] NTIA. U.S. Frequency Allocation Chart Available: http://www.ntia.doc.gov/files/ntia/publications/2003-allochrt.pdf

[5] FCC. (2002). Report of the Spectrum Efficiency Working Group Available: http://transition.fcc.gov/sptf/files/SEWGFinalReport 1.pdf

[6] J. Mitola, III and G. Q. Maguire, Jr., "Cognitive radio: making software radios more personal," Personal Communications, IEEE, vol. 6, pp. 13-18, 1999. 
[7] M. Darvishi, R. van der Zee, and B. Nauta, "Design of Active N-Path Filters," Solid-State Circuits, IEEE Journal of, vol. 48, pp. 2962-2976, Dec. 2013.

[8] L. E. Franks and I. W. Sandberg, "An Alternative Approach to the Realization of Network Transfer Functions: The N-Path Filters," Bell Sys. Tech. J., vol. 39, pp. 1321-1350, Sep. 1960.

[9] C. Andrews and A. C. Molnar, "A Passive Mixer-First Receiver With Digitally Controlled and Widely Tunable RF Interface," Solid-State Circuits, IEEE Journal of, vol. 45, pp. 2696-2708, 2010.

[10] A. Ghaffari, E. A. M. Klumperink, M. C. M. Soer, and B. Nauta, "Tunable High-Q N-Path Band-Pass Filters: Modeling and Verification," Solid-State Circuits, IEEE Journal of, vol. 46, pp. 998-1010, 2011.

[11] M. Darvishi, R. van der Zee, E. A. M. Klumperink, and B. Nauta, "Widely Tunable 4th Order Switched Gm-C Band-Pass Filter Based on N-Path Filters," Solid-State Circuits, IEEE Journal of, vol. 47, pp. 3105-3119, 2012.

[12] D. Ruffieux, J. Chabloz, M. Contaldo, C. Muller, F. Pengg, P. Tortori, et al., "A narrowband multi-channel $2.4 \mathrm{GHz}$ MEMS-based transceiver," SolidState Circuits, IEEE Journal of, vol. 44, pp. 228-239, Jan. 2009.

[13] S. Razafimandimby, C. Tilhac, A. Cathelin, A. Kaiser, and D. Belot, "An electronically tunable bandpass BAW-filter for a zero-IF WCDMA receiver," in Eur. Solid-State Circuits Conf. (ESS-CIRC), 2006, pp. 142-145.

[14] H. van de Vel, J. Briaire, C. Bastiaansen, P. Van Beek, G. Geelen, H. Gunnink, et al., "A $240 \mathrm{~mW} 16 \mathrm{~b} 3.2 \mathrm{GS} / \mathrm{s}$ DAC in $65 \mathrm{~nm}$ CMOS with $<-80 \mathrm{dBc}$ IM3 up to 600MHz," in ISSCC Dig. Tech. Papers, 2014, pp. 206-207.

[15] G. Engel, S. Kuo, and Rose, "A 14b 3/6GS/s Current-Steering RF DAC in 0.18um CMOSwith 66dB ACLRat 2.9GHz," in ISSCC Dig. Tech Papers, 2012, pp. 458-459.

[16] E. Olieman, A. J. Annema, and B. Nauta, "A $110 \mathrm{~mW}, 0.04 \mathrm{~mm}^{2}, 11 \mathrm{GS} / \mathrm{s} 9-$ bit interleaved DAC in 28nm FDSOI with $>50 \mathrm{~dB}$ SFDR across Nyquist," in Symposium on VLSI Circuits, (accepted for publication) Honolulu, Hawaii, USA, 2014.

[17] B. Razavi, RF Microelectronics. Upper Saddle River, NJ: Prentice Hall, 1998.

[18] R. Shrestha, E. A. M. Klumperink, E. Mensink, G. J. M. Wienk, and B. Nauta, "A Polyphase Multipath Technique for Software-Defined Radio Transmitters," Solid-State Circuits, IEEE Journal of, vol. 41, pp. 2681-2692, 2006.

[19] E. Mensink, E. A. M. Klumperink, and B. Nauta, "Distortion cancellation by polyphase multipath circuits," IEEE Trans. Circuits Syst. II, Exp. Briefs, vol. 57, pp. 1785-1794, Sep. 2005. 
[20] E. A. M. Klumperink, S. M. Louwsma, G. J. M. Wienk, and B. Nauta, "A CMOS switched transconductor mixer," Solid-State Circuits, IEEE Journal of, vol. 39, pp. 1231-1240, 2004.

[21] FCC, "Second Report and Order and Memorandum Opinion and Order In the Matter of Unlicensed Operation in the TV Broadcast Bands, Additional Spectrum for Unlicensed Devices Below $900 \mathrm{MHz}$ and in the $3 \mathrm{GHz}$ Band," Nov. 2008.

[22] FCC, "In the Matter of Unlicensed Operation in the TV Broadcast Bands Additional Spectrum for Unlicensed Devices Below $900 \mathrm{MHz}$ and in the 3GHz Band," Sep. 2010. 


\section{CHAPTER TWO}

\section{Transmitter Architectures}

There are various transmitter architectures which are targeted towards software defined or a cognitive radio application. Aims vary; where some design focus on wideband linearity or on issues like wideband LO generation, flexible digital image filtering or power control. A few of the architectures aim for suppression or flexible filtering of LO harmonics. In this chapter a brief overview is given of some of these transmitter architectures in recent literature, focussing on multimode/ multi-standard aspects of their design. In section 2.1, the transmitter architectures which claim to be multimode and targeted towards software defined radio applications are discussed. These architectures do not aim for harmonic suppression. The transmitter architectures which specifically aim for harmonic suppression are discussed in section 2.2. In section 2.3, the out of band emission requirements of a Cognitive Radio Transmitter are also discussed.

\subsection{Multimode Transmitter Architectures Review}

Transmitters which claim to be multimode have the flexibility to cater to various baseband bandwidths and modulation standards. A multimode transmitter based on the digital to RF converter (DRFC) architecture [1] shown in Figure 2.1 focuses on making the digital baseband flexible and also flexibility in the choice of frequency. This is accomplished by removing the analog baseband filter and as the name suggests directly converts the digital baseband to RF. The filtering is accomplished via oversampling which can be adapted. By changing the oversampling ratio for different baseband bandwidths; the digital images and noise can be suppressed, depending on how high the oversampling ratio is chosen. As shown in Figure 2.2, in the DRFC architecture [1] there is no analog baseband and the digital bits $\left(\mathrm{D}_{1}, \mathrm{D}_{2}, \ldots \mathrm{D}_{\mathrm{N}}\right)$ are converted to the analog domain and simultaneously upconverted via the LO to the RF output. The DAC function is therefore built into the DRFC. Improvement in the Image rejection ratio and LO leakage are also claimed because of improved matching of the I and Q paths of Figure 2.1. An RF filter would still be required to suppress the harmonics of the LO. 


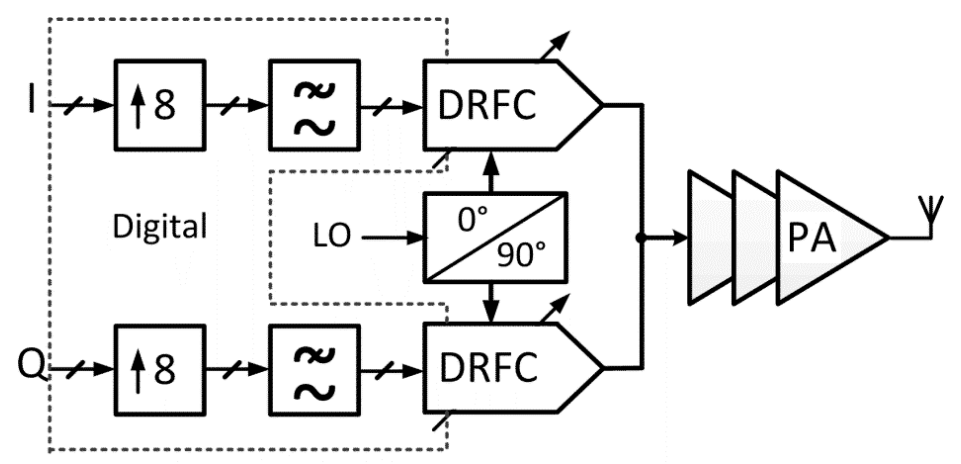

Figure 2.1 Transmitter architecture based on the Digital to RF converter[1].

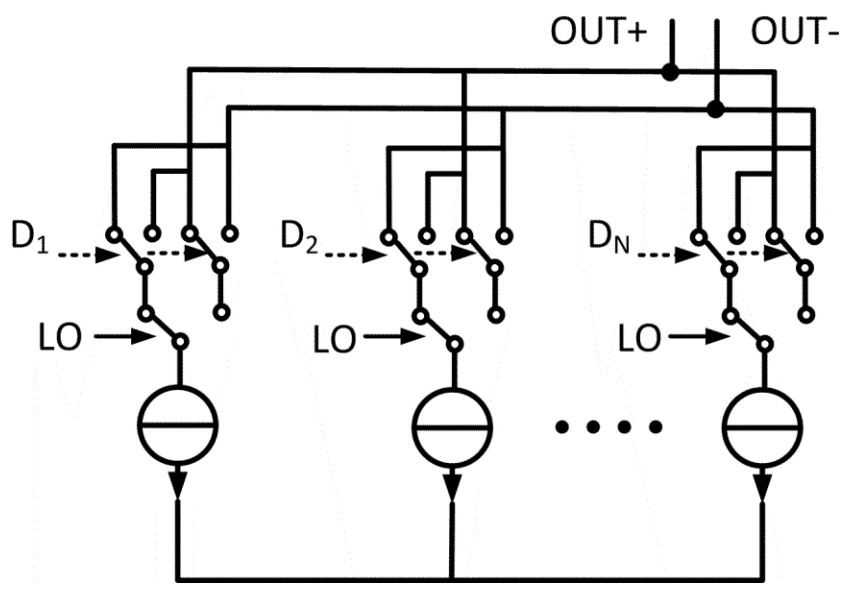

Figure 2.2. Digital to RF converter (DRFC) [1]

In [2], a multimode transmitter based on a direct quadrature voltage modulator (DQVM) in combination with a highly oversampled baseband is proposed shown in Figure 2.3. Here the oversampled baseband in combination with a relaxed low pass filter (LPF) attenuates the DAC quantization noise and DAC images (see section 1.2.6 and 1.2.7). The passive mixer is used to gain linearity benefits, i.e. achieve low EVM (see section 1.2.1 and 1.2.2). Here also an RF filter is required if the LO harmonics are to be suppressed. In [3-5] a digital FIR filter embedded with a digital to RF converter is used to suppress quantization noise. In [6] a reconfigurable baseband path design is presented which can process WLAN, Bluetooth and UMTS signals, thanks to a digitally programmable filter and DAC sampling frequency. In [7] a 17 bit RFDAC is employed in a polar transmitter architecture, aiming to lower far out noise and thus supporting both $2 \mathrm{G}$ and $3 \mathrm{G}$ standards. The WCDMA transmitter in [8] also focuses on reducing the upconverter noise and thus removing the transmitter SAW filter. The passive mixer [9] based $0.1-3 \mathrm{GHz}$ upconverter in [10] also focuses on reducing 
the upconverted baseband noise by employing programmable low pass filters before the voltage-sampling mixer and avoids the inter-stage SAW filter in FDD operation. An all digital PLL (ADPLL) [11] based polar Transmitter [12] for EDGE presents a highly digital design which meets the spectral mask requirements without requiring SAW filters. A recent wideband all-Digital I/Q based RFDAC implementation [13] has reported high efficiency $(42 \%)$ at an output power of $22.8 \mathrm{dBm}$ while achieving a wide baseband bandwidth (154 $\mathrm{MHz}$.

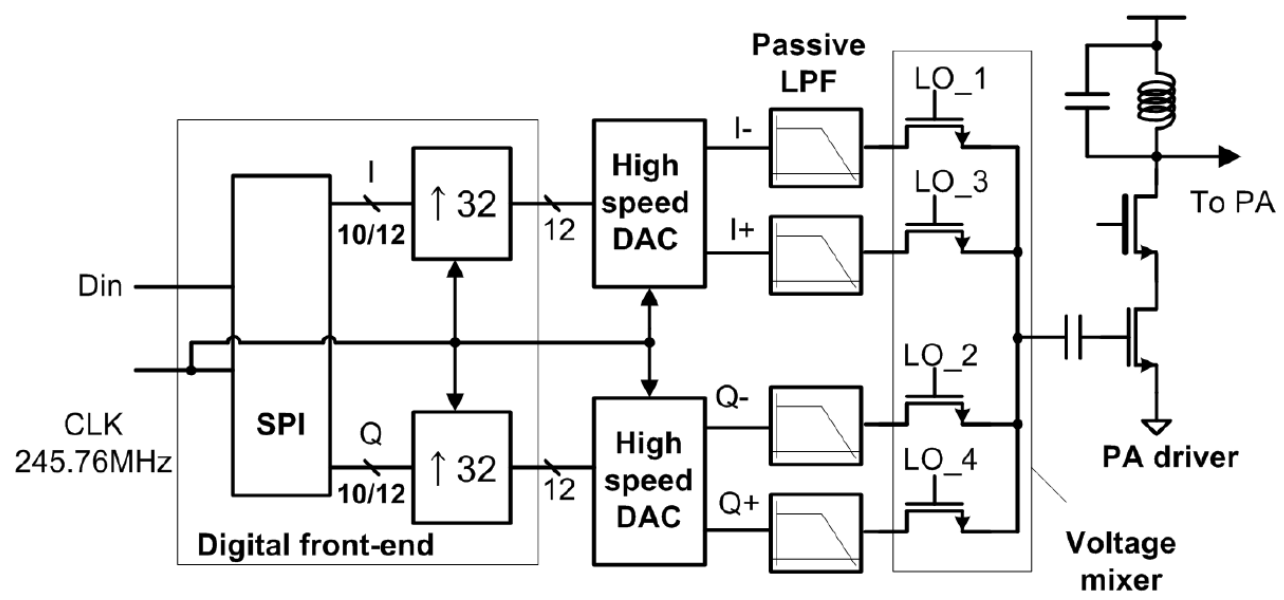

Figure 2.3. Transmitter architecture based on the direct quadrature voltage modulator [9]

The transmitter architecture in [14] uses a digital to RF upconversion but also incorporates a fourth order tunable LC RF band-pass filter to suppress the spurs associated with the digital to RF upconversion. This filter provides a suppression of around $30 \mathrm{~dB}$ at $1 \mathrm{GHz}$ offset from the centre frequency, but it has a limited tuning range of $\pm 8 \%$ at $5.25 \mathrm{GHz}$ which is not very flexible for wideband operation. The SDR transmitter architecture of [15] operates over a frequency range of $100 \mathrm{MHz}$ to $2.5 \mathrm{GHz}$, mainly focusing on making the baseband path flexible and provides linearization for narrow and medium band protocols. It uses a flexible direct digital synthesizer to drive the switching mixers and provides fast cycle-to-cycle frequency switching.

In all the multiband multimode transmitter architectures mentioned above none of them focus on wideband local oscillator (LO) harmonic suppression or cancellation. If the cognitive radio transmitter has to be flexible and cover a wide range of radio frequencies, without dedicated bulky RF band-pass filters, the techniques mentioned in the next section can be potential candidates. 


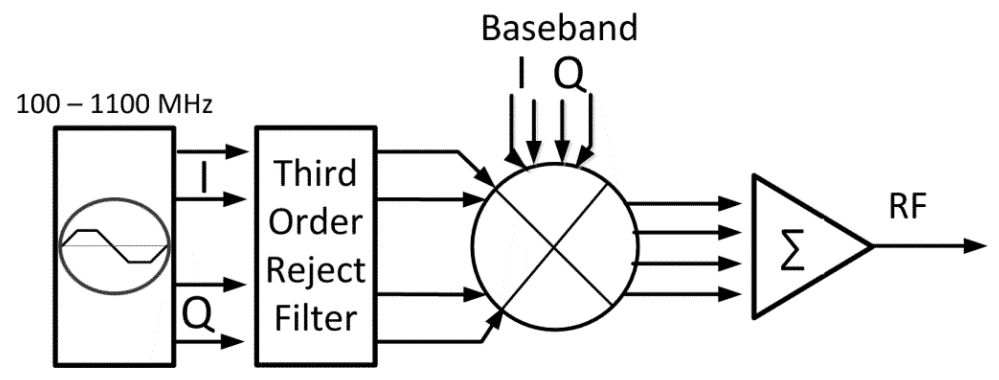

Figure 2.4. Transmitter architecture based on making the LO sinusoidal [16].

\subsection{Harmonic and Distortion Suppression Techniques}

In literature we found three main approaches to address the problem of LO harmonics. One approach exploits a sinewave like LO [16] driving a linear multiplier, shown in Figure 2.4. In an attempt to make the LO more sinusoidal the third harmonic of the square wave LO is rejected. However, a high linearity analog multiplier design is challenging, while providing only modest output power compared to switching mixers. Also, flexible wideband sinewave LO-generation is non-trivial and the LO-amplitude is critical as it should not drive the LOinput of the multiplier into its non-linear region.

A second approach is to use switching mixers which do produce LO-harmonics, but cancel harmonics via multiple mixer paths exploiting different phases [17-23] or different phase and amplitude [24-26]. The harmonic rejection technique proposed in [24] is used in many wideband SDR receivers [27-29], to suppress harmonic down-conversion. In a 2-stage implementation, more than $60 \mathrm{~dB}$ harmonic rejection can be achieved [30]. The transmitters in $[25,26]$ are also based on the same principle. This principle is shown in Figure 2.5, in comparison to a conventional switching mixer, the harmonic rejection mixer (HRM) allows for cancelling of the third and fifth harmonics of the LO waveform, because of their antiphase addition at the mixer output. Figure 2.6a shows the resultant staircase approximation of a sinewave generated due to the multiphase LO, while Figure 2.6b shows the vector diagram of the harmonics at the output of mixer. As shown one of the phases require a weighting factor of $\sqrt{2}$. Making this weighting factor in circuit design with good accuracy can be a challenge, but has been shown to be possible by using a two stage harmonic rejection technique introduced in [30], and also recently used for a cognitive radio transmitter in [26]. It should be noted that in the HRM technique the baseband signal has the same phase for all the mixers, and the technique only focuses on suppressing the LO harmonics. If the multiple baseband phases are also generated along with multiple LO 
phases, distortion suppression benefits can also be obtained, as described by the polyphase multipath technique [17], a brief overview of which is presented in the next subsection.
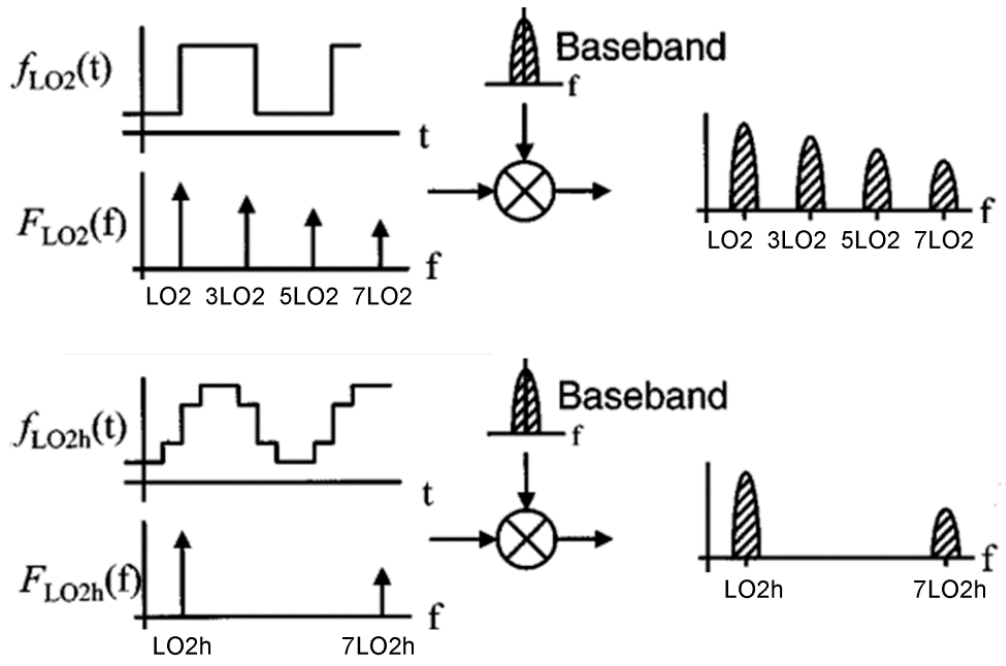

Figure 2.5. Conventional switching mixer (top) Harmonic Rejection Principle (bottom) [24]

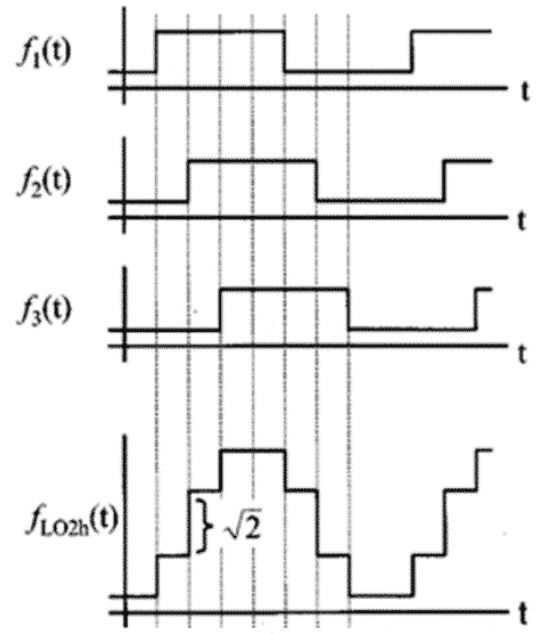

(a) 1st and 7th harmonic add up

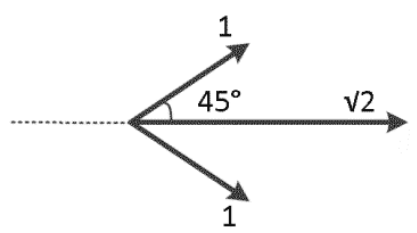

$3^{\text {rd }}$ and $5^{\text {th }}$ harmonic are cancelled



(b)

Figure 2.6. (a)Generation of the Harmonic Rejection LO waveform[24].(b) Vector diagram of the harmonics at the output.

A third way to clean the transmitter spectrum obviously is to apply filters. However, frequency agile transmitters would require flexibly tunable RF filters, which are difficult to implement especially for high Q. Passive LC filters are linear but high-Q inductors are 
problematic certainly at low RF frequency and require large chip area. Active filter techniques can be used to suppress higher order harmonics [25], but handling sufficient power at high linearity is a problem. On the other hand, low-Q passive RC filters are suitable for on chip integration as well as being linear and power efficient, but generally do not provide enough suppression. A combination of a passive RC filter and the polyphase multipath technique is proposed in [22],[23] and described in Chapter 3. It is shown that this combination can provide harmonic suppression with less complexity and power consumption.

\subsubsection{Polyphase Multipath Technique}

When a non-linear circuit is excited by a sinusoidal input having a frequency $\omega$, its output spectrum not only contains the frequency component at $\omega$ but also multiples of this frequency component at $2 \omega, 3 \omega$ and so on. These higher order terms are the unwanted distortion components. The Polyphase multipath technique [17-23] is aimed at cancelling these higher order distortion terms. Figure 2.7 shows such an n-path circuit. The idea is to divide the nonlinear circuit into $n$ equal smaller slices and apply equal but opposite phase shifts before and after each nonlinear circuit. In the remainder of this section an explanation is given of the technique. The equations are taken from [19] and the reader is referred to [17] for more background information.

If the phase shift in path $i$ is $(i-1) \times \varphi$, where $\varphi$ is a phase shift constant satisfying $n \times \varphi=360^{\circ}$, the output of the multipath circuit would produce the desired harmonic and cancel many of the higher order terms. This can be seen as follows: If the signal $x(t)=\cos (\omega t)$ is applied as input to a weakly nonlinear system, the output of the $i^{\text {th }}$ path can be written as:

$$
\begin{aligned}
\mathrm{p}_{\mathrm{i}}(\mathrm{t})=\mathrm{b}_{0}+\mathrm{b}_{1} \cdot \cos (\omega \mathrm{t}+(\mathrm{i}-1) \varphi) & \\
& +\mathrm{b}_{2} \cdot \cos (2 \omega \mathrm{t}+2(\mathrm{i}-1) \varphi)+\mathrm{b}_{3} \cdot \cos (3 \omega \mathrm{t}+3(\mathrm{i}-1) \varphi) \\
& +\ldots
\end{aligned}
$$

where $b_{0}, b_{1}, b_{2} b_{3} \ldots$ are constants. From (2.1), it can be seen that the phase of the $k^{\text {th }}$ harmonic at the output of the nonlinear circuit rotates by $\mathrm{k}$ times the input phase $(i-1) \varphi$. The phase shifters $-(i-1) \varphi$, after the nonlinear blocks are required to align the fundamental components at $\omega$ in phase again. The output of these phase shifters can be written as

$$
y_{i}(t)=b_{0}+b_{1} \cdot \cos (\omega t)+b_{2} \cdot \cos (2 \omega t+(i-1) \varphi)+b_{3} \cdot \cos (3 \omega t+2(i-1) \varphi)+\ldots
$$




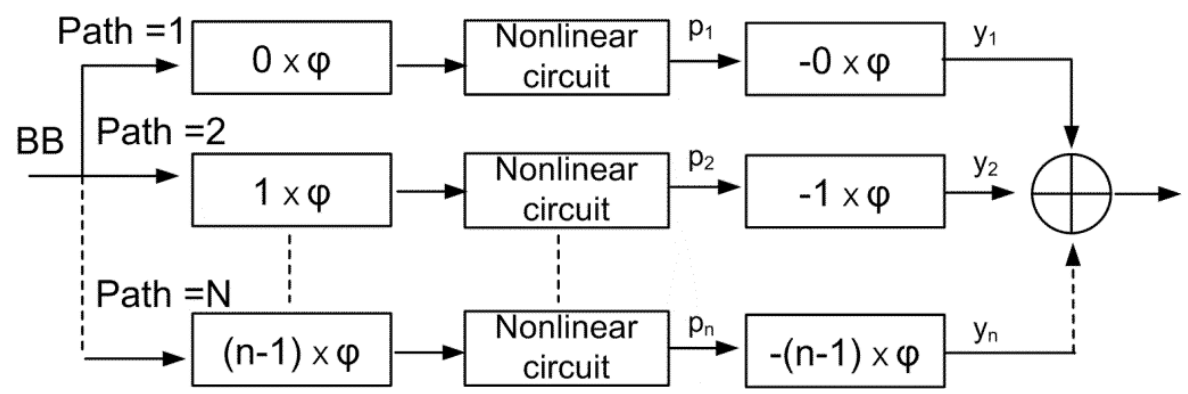

Figure 2.7. Polyphase $n$ path circuit [19]

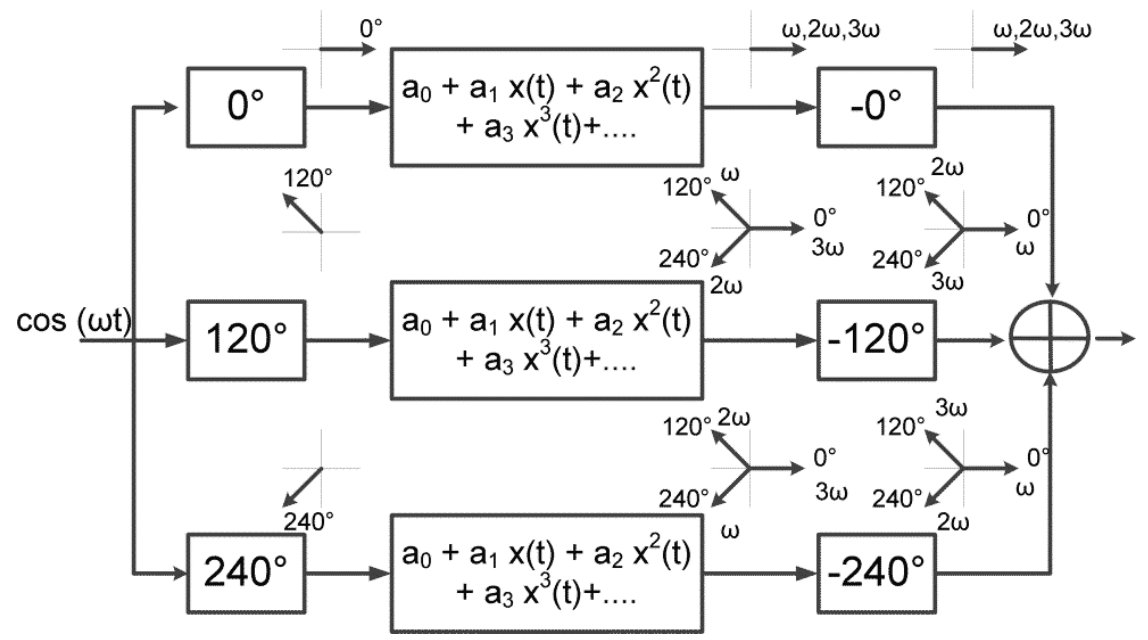

Figure 2.8. Polyphase 3 path system [19]

In (2.2), the phase of the fundamental component is identical for all the paths, but the phases of the harmonics are different for each path. If the phase is chosen such that, $\phi=\frac{360^{\circ}}{n}$, then the higher order terms are cancelled except for the harmonics that satisfy the following equation.

$$
k=j \times n+1
$$

Where $\mathrm{j}=0,1,2,3 \ldots$ The well known differential circuit also exploits such a harmonic cancellation but it only cancels the even order terms. A three path system is shown in Figure 2.8. In this scheme phase shifts of $0^{\circ}, 120^{\circ}$ and $240^{\circ}$ are added before and equal but opposite phases after the nonlinear element. As a result the fundamental components add up 


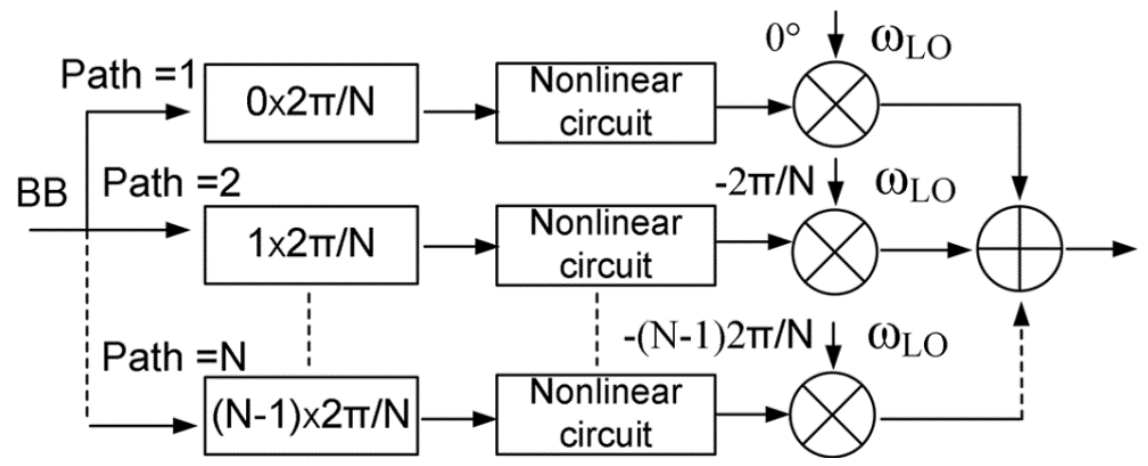

Figure 2.9. Phase shifts after nonlinear circuit replaced by mixers [19]

in phase while the phases of the second and third harmonics cancel each other out. The fourth harmonic would again have the same phase before summation and would not be cancelled. So the first non-cancelled harmonic in an $n$ path system would be the $(\mathrm{n}+1)^{\text {th }}$ harmonic.

In case of two tones $\omega_{1}$ and $\omega_{2}$ as inputs to the system, the phase shift of the $\mathrm{p} \cdot \omega_{1}+\mathrm{q} \cdot \omega_{2}$ products ( $\mathrm{p}$ and $\mathrm{q}$ are integers) at the output of the $i^{\text {th }}$ path will be $(p+q-1) \times(i-1) \varphi$. So the products which satisfy $(2.4)$ will not be cancelled, where $\mathrm{j}=0,1,2,3 \ldots$.

$$
p+q=j \times n+1
$$

The second set of phase shifts can be implemented via mixers as shown in Figure 2.9 [19]. Wideband phase shifters are difficult to implement but mixers can transfer the phase information at the LO port to the RF output. The first set of phase shifts in the baseband is the subject of research in section 4.1. Now there are two input ports (BB and LO) as compared to Figure 2.7, so a slightly different equation will result as compared to (2.3). According to [19] spectral components at $\mathrm{k}_{\mathrm{LO}} \omega_{\mathrm{LO}}+m \omega_{\mathrm{BB}}$ are generated by a single-path upconversion, where $k_{L O}$ is the $\mathrm{k}^{\text {th }}$ harmonic of the LO frequency $\omega_{\mathrm{LO}}, m$ is a positive or negative integer, and $\omega_{\mathrm{BB}}$ is the single tone $\mathrm{BB}$ frequency. For an N-path upconversion many spectral components can be cancelled, except if [19]:

$$
k_{L O}=j \times N+m
$$

where $j=\ldots-2,-1,0,1,2 \ldots$ 

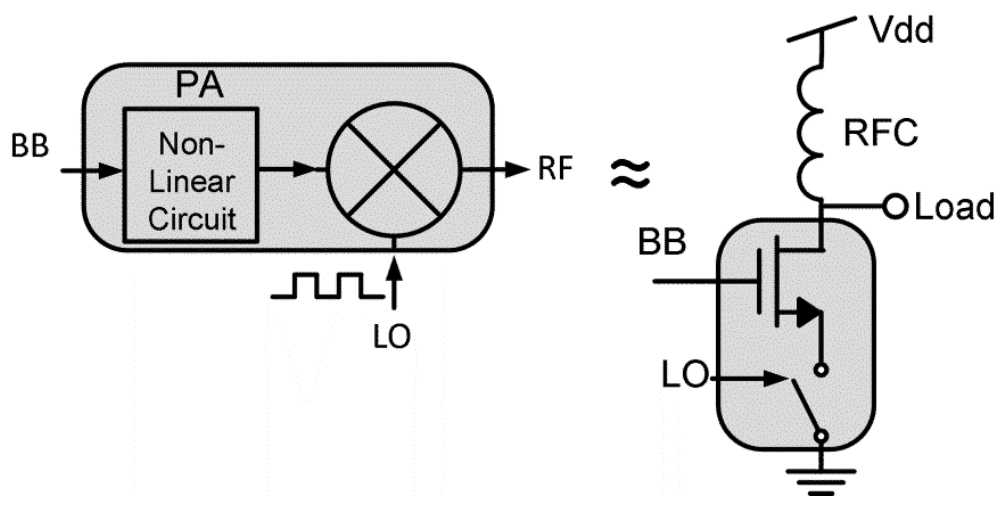

Figure 2.10 Power Upconverter (PU) used in [19]

The combination of the functionality of the power amplifier and upconverter is termed as a Power upconverter (PU) in [19]. The circuit to describe this functionality is shown in Figure 2.10. Here the PA is a single transistor operating as a V-I converter, switched on and off by a switch driven by the LO. The V-I conversion and upconversion is therefore done in the same circuit via the switched trans-conductor [31]. In this thesis, the term power upconverter (PU) or just upconverter will be used to describe this circuit.

An 18-path implementation of the polyphase multipath technique for a flexible transmitter architecture [19] has shown that it can cancel a large number of harmonics, distortion and sideband products resulting from a power upconversion (PU). However the technique required a lot of power to generate the 18 LO phases, while achieving $40 \mathrm{~dB}$ harmonic suppression upto the first uncancelled harmonic. The technique which is the subject of this thesis proposed in [22] and discussed in Chapter 3 allows for achieving similar suppression for ALL the harmonics, but with lesser paths and lower power consumption.

The multipath technique has also been exploited to cancel distortion products in a Digital to Analog conversion process [32] and also in a sine wave frequency synthesizer [33]. A modification of the technique has also been proposed [34], but it lacks image rejection. The inter-modulation products remain un-cancelled and are not cancelled by this technique [17, 19], however digital pre-distortion [35] applied to the multipath architecture allows for suppression of these terms.

The harmonic rejection mixers used in [24] and the switched transconductor mixers [31] used in polyphase multipath upconversion [19] can achieve high output power as they can operate in saturation as compared to the mixers operating in their linear region [16]. In order to achieve enough suppression multiple accurate phases of the LO and/or the baseband have to be generated, but digital clocks can be used. Flexibly programmable 
digital frequency dividers can be exploited, enabling software defined and cognitive radios to benefit from Moore's law. Still, there are limits to the number of phases that can be realized at high frequency, while phase accuracy and power dissipation is also a concern $[36,37]$.

From the discussion above, we conclude that the multi-path mixer techniques exploiting digital square-wave LO-paths have the most attractive properties for agile dynamic spectrum access. In [24-26], harmonic rejection is achieved using different LO-phases and amplitude weighting, sharing one baseband signal. If multiple baseband phases are also generated, we can realize a Polyphase Multipath up-converter and now, not only harmonics are cancelled, but also many distortion and side-band products [17, 19]. In other words: apart from harmonic rejection mixing, linearity benefits are also achieved.

\subsection{Cognitive Radio transmitter requirements}

Regulators around the world are opening up the RF spectrum for devices that can operate where-ever there is free spectrum available in a certain RF band [38, 39]. In order to meet regulatory requirements it is crucial that these devices do not interfere with incumbent users of the frequency spectrum. As shown in [40], one of the challenges for a cognitive radio transmitter is that the out of band emissions (OOB) in the adjacent channels and beyond the adjacent channels have to be less than $55 \mathrm{~dB}$ and $53 \mathrm{~dB}$ respectively relative to the desired (maximum) signal power of $20 \mathrm{dBm}$ [38]. In terms of absolute power this means that the OOB emissions should be $\leq-33 \mathrm{dBm}$ ( $5 \mathrm{MHz}$ signal bandwidth assumed as in [40]). If the maximum signal power is in the range of $0-10 \mathrm{dBm}$, which can be enough for a portable device, the OOB emissions should then be $33 \mathrm{~dB}-43 \mathrm{~dB}(+10 \mathrm{dBm}-(-33 \mathrm{dBm})=43 \mathrm{~dB})$ respectively below the desired signal. A more recent report from FCC [39] requires that out of band emissions in the adjacent channel to be better than $-38 \mathrm{dBm}$ (in $6 \mathrm{MHz}$ ), while the maximum desired signal power specified is $17 \mathrm{dBm}$. On a relative scale this requirement implies that the out of band emissions should be $55 \mathrm{~dB}$ below the desired signal. If the signal to be transmitted has an output power of $0-10 \mathrm{dBm}$, the $\mathrm{OOB}$ emission requirement on a relative scale comes out to be $38 \mathrm{~dB}-48 \mathrm{~dB}(+10 \mathrm{dBm}-(-38 \mathrm{dBm})=48 \mathrm{~dB})$ respectively below the desired signal. In essence if the output power to be transmitted is less, the OOB emission requirements can also be reduced from their maximum values. At the maximum output power levels the OOB requirements are also the toughest. 


\subsection{Conclusions}

In literature there are many transmitter architectures which are focused towards multimode or multi-standard operation for a SDR/ Cognitive radio Transmitter. Most of them focus on making the baseband design flexible, relaxing or removing the baseband filtering, improving the linearity and/or noise performance of the transmitter. A few of the transmitter architectures focus on removing or suppressing the transmitted signals at and around the harmonics of the local oscillator. The harmonic rejection mixer technique, which requires multiphase LO along with amplitude weighting of one of the paths has attractive properties for LO harmonic cancellation but doesn't have distortion cancellation properties. However, if multiple baseband phases are also generated along with a multiphase LO, linearity benefits are also achieved as is the case with the Polyphase Multipath Upconversion. This can be very beneficial in a dynamic spectrum access environment. From the FCC reports on spectrum utilization for the unlicensed devices in the TV bands, it is seen that that the out of band emission in the $6 \mathrm{MHz}$ bandwidth are required to be $55 \mathrm{~dB}$ below the desired signal. The out of band emission requirement is relaxed if the output power requirement is reduced.

\section{References}

[1] P. Eloranta, P. Seppinen, S. Kallioinen, T. Saarela, and A. Parssinen, "A Multimode Transmitter in 0.13 um CMOS Using Direct-Digital RF Modulator," Solid-State Circuits, IEEE Journal of, vol. 42, pp. 2774-2784, Dec 2007.

[2] X. He, J. van Sinderen, and R. Rutten, "A 45nm WCDMA Transmitter Using Direct Quadrature Voltage Modulator with High Oversampling Digital Front-End," in International Solid State Circuits Conference, 2010.

[3] S. M. Taleie, Y. Han, T. Copani, B. Bakkaloglu, and S. Kiaei, "A 0.18um CMOS Fully Integrated RFDAC and VGA for WCDMA Transmitters," in IEEE Radio Frequency Integrated circuits (RFIC) Symposium, 2008.

[4] W. M. Gaber, P. Wambacq, J. Craninckx, and M. Ingels, "A CMOS IQ direct digital RF modulator with embedded RF FIR-based quantization noise filter," in ESSCIRC (ESSCIRC), 2011 Proceedings of the, 2011, pp. 139-142.

[5] S. Fukuda, S. Miya, M. Io, K. Hamashita, and B. Nauta, "Direct-Digital modulation (DIDIMO) Transmitter with $-156 \mathrm{dBc} / \mathrm{Hz}$ Rx-Band Noise using 
FIR structure," in Proc. European Solid-State Circuits Conf. (ESSCIRC), 2012, pp. 53-56.

[6] N. Ghittori, A. Vigna, P. Malcovati, S. D'Amico, and A. Baschirotto, "1.2-V Low-Power Multi-Mode DAC+Filter Blocks for Reconfigurable (WLAN/UMTS, WLAN/Bluetooth) Transmitters," Solid-State Circuits, IEEE Journal of, vol. 41, pp. 1970-1982, Sep. 2006.

[7] Z. Boos, A. Menkhoff, F. Kuttner, M. Schimper, J. Moreira, H. Geltinger, et al., "A fully digital multimode polar transmitter employing 17b RF DAC in $3 \mathrm{G}$ mode," in Solid-State Circuits Conference Digest of Technical Papers (ISSCC), 2011 IEEE International, 2011, pp. 376-378.

[8] Q. Huang, J. Rogin, X. Chen, D. Tschopp, T. Burger, T. Christen, et al., "A tri-band SAW-less WCDMA/HSPA RF CMOS transceiver with on-chip DCDC converter connectable to battery," in Solid-State Circuits Conference Digest of Technical Papers (ISSCC), 2010 IEEE International, 2010.

[9] X. He and J. V. Sinderen, "A Low-Power, Low-EVM, SAW-Less WCDMA Transmitter Using Direct Quadrature Voltage Modulation," Solid-State Circuits, IEEE Journal of, vol. 44, pp. 3448-3458, Dec. 2009.

[10] M. Ingels, V. Giannini, J. Borremans, G. Mandal, B. Debaillie, P. Van Wesemael, et al., "A $5 \mathrm{~mm}^{2}$ 40nm LP CMOS 0.1-to-3GHz multistandard transceiver," in Solid-State Circuits Conference Digest of Technical Papers (ISSCC), 2010 IEEE International, 2010.

[11] R. B. Staszewski, J. L. Wallberg, S. Rezeq, C.-M. Hung, O. E. Eliezer, S. K. Vemulapalli, et al., "All-digital PLL and transmitter for mobile phones," Solid-State Circuits, IEEE Journal of, vol. 40, pp. 2469-2482, Dec. 2005.

[12] J. Mehta, R. B. Staszewski, O. Eliezer, S. Rezeq, K. Waheed, M. Entezari, et al., "A $0.8 \mathrm{~mm}^{2}$ all-digital SAW-less polar transmitter in 65nm EDGE SoC," in Solid-State Circuits Conference Digest of Technical Papers (ISSCC), 2010 IEEE International, 2010, pp. 58-59.

[13] M. S. Alavi, R. B. Staszewski, L. C. N. de Vreede, and J. R. Long, "A Wideband 2 × 13-bit All-Digital I/Q RF-DAC," Microwave Theory and Techniques, IEEE Transactions on, vol. 62, pp. 732-752, Apr. 2014.

[14] A. Jerng and C. G. Sodini, "A Wideband Digital-RF Modulator for High Data Rate Transmitters," Solid-State Circuits, IEEE Journal of, vol. 42, pp. 1710-1722, Aug 2007.

[15] G. Cafaro, T. Gradishar, J. Heck, S. Machan, G. Nagaraj, S. Olson, et al., "A $100 \mathrm{MHz}-2.5 \mathrm{GHz}$ Direct Conversion CMOS Transceiver for SDR Applications," in Radio Frequency Inegrated Circuits (RFIC) Symposium, 2007.

[16] M. A. F. Borremans, C. R. C. De Ranter, and M. S. J. Steyaert, "A CMOS dual-channel, $100-\mathrm{MHz}$ to $1.1-\mathrm{GHz}$ transmitter for cable applications," Solid-State Circuits, IEEE Journal of, vol. 34, pp. 1904-1913, 1999. 
[17] E. Mensink, E. A. M. Klumperink, and B. Nauta, "Distortion cancellation by polyphase multipath circuits," IEEE Trans. Circuits Syst. II, Exp. Briefs, vol. 57, pp. 1785-1794, Sep. 2005.

[18] E. Mensink, E. A. M. Klumperink, and B. Nauta, "Distortion cancellation via polyphase multipath circuits," in Circuits and Systems, 2004. ISCAS '04. Proceedings of the 2004 International Symposium on, 2004, pp. I-1098101 Vol.1.

[19] R. Shrestha, E. A. M. Klumperink, E. Mensink, G. J. M. Wienk, and B. Nauta, "A Polyphase Multipath Technique for Software-Defined Radio Transmitters," Solid-State Circuits, IEEE Journal of, vol. 41, pp. 2681-2692, 2006.

[20] E. Klumperink, R. Shrestha, E. Mensink, G. Wienk, Z. Ru, and N. Bram, "Multipath Polyphase Circuits and their Application to RF Transceivers," in Circuits and Systems, 2007. ISCAS 2007. IEEE International Symposium on, 2007, pp. 273-276.

[21] E. A. M. Klumperink, R. Shrestha, E. Mensink, V. J. Arkesteijn, and B. Nauta, "Cognitive radios for dynamic spectrum access - polyphase multipath radio circuits for dynamic spectrum access," Communications Magazine, IEEE, vol. 45, pp. 104-112, 2007.

[22] S. Subhan, E. A. M. Klumperink, and B. Nauta, "Towards suppression of all harmonics in a polyphase multipath transmitter," in Circuits and Systems (ISCAS), 2011 IEEE International Symposium on, 2011, pp. 2185-2188.

[23] S. Subhan, E. A. M. Klumperink, A. Ghaffari, G. J. M. Wienk, and B. Nauta, "A 100-800 MHz 8-Path Polyphase Transmitter With Mixer Duty-Cycle Control Achieving < $-40 \mathrm{dBc}$ for ALL Harmonics," Solid-State Circuits, IEEE Journal of, vol. 49, pp. 595-607, Mar. 2014.

[24] J. A. Weldon, R. S. Narayanaswami, J. C. Rudell, L. Li, M. Otsuka, S. Dedieu, et al., "A 1.75-GHz highly integrated narrow-band CMOS transmitter with harmonic-rejection mixers," Solid-State Circuits, IEEE Journal of, vol. 36, pp. 2003-2015, Dec. 2001.

[25] K. Jongsik, L. Seung Jun, K. Seungsoo, H. Jong Ok, E. Yun Seong, and S. Hyunchol, "A 54-862-MHz CMOS Transceiver for TV-Band White-Space Device Applications," Microwave Theory and Techniques, IEEE Transactions on, vol. 59, pp. 966-977, 2011.

[26] K. Un, M. P., and R. P. Martins, "A 53-to-75-mW, 59.3-dB HRR, TV-Band White-Space Transmitter Using a Low-Frequency Reference LO in 65-nm CMOS," Solid-State Circuits, IEEE Journal of, vol. 48, pp. 2078-2089, Sep 2013.

[27] R. Bagheri, A. Mirzaei, M. E. Heidari, S. Chehrazi, L. Minjae, M. Mikhemar, et al., "An 800-MHz-6-GHz Software-Defined Wireless Receiver in 90-nm 
CMOS," Solid-State Circuits, IEEE Journal of, vol. 41, pp. 2860-2876, Dec.2006.

[28] S. Lerstaveesin, M. Gupta, D. Kang, and B.-S. Song, "A 48-860 MHz CMOS Low -IF Direct-Conversion DTV Tuner," Solid-State Circuits, IEEE Journal of, vol. 43, pp. 2013-2024, Sep. 2008.

[29] F. Gatta, R. Gomez, Y. J. Shin, T. Hayashi, and et.al, "An Embedded $65 \mathrm{~nm}$ CMOS Baseband IQ 48 MHz-1 GHz Dual Tuner for DOCSIS 3.0," Solid-State Circuits, IEEE Journal of, vol. 44, pp. 3511-3525, Dec. 2009.

[30] Z. Ru, N. A. Moseley, E. Klumperink, and B. Nauta, "Digitally Enhanced Software-Defined Radio Receiver Robust to Out-of-Band Interference," Solid-State Circuits, IEEE Journal of, vol. 44, pp. 3359-3375, 2009.

[31] E. A. M. Klumperink, S. M. Louwsma, G. J. M. Wienk, and B. Nauta, "A CMOS switched transconductor mixer," Solid-State Circuits, IEEE Journal of, vol. 39, pp. 1231-1240, 2004.

[32] G. L. Radulov, P. J. Quinn, P. Harpe, H. Hegt, and A. Van Roermund, "Parallel current-steering D/A Converters for Flexibility and Smartness," in Circuits and Systems, 2007. ISCAS 2007. IEEE International Symposium on, 2007, pp. 1465-1468.

[33] W. A. Ling and P. P. Sotiriadis, "A Nearly All-Digital Frequency Mixer Based on Nonlinear Digital-to-Analog Conversion and Intermodulation Cancellation," Circuits and Systems I: Regular Papers, IEEE Transactions on, vol. 58, pp. 1695-1704, 2011.

[34] E. A. Sobhy and S. Hoyos, "A Multiphase Multipath Technique With Digital Phase Shifters for Harmonic Distortion Cancellation," Circuits and Systems II: Express Briefs, IEEE Transactions on, vol. 57, pp. 921-925, 2010.

[35] Y. Xi, D. Chaillot, P. Roblin, L. Wan-Rone, L. Jongsoo, P. Hyo-Dal, et al., "Poly-Harmonic Modeling and Predistortion Linearization for SoftwareDefined Radio Upconverters," Microwave Theory and Techniques, IEEE Transactions on, vol. 58, pp. 2125-2133, 2010.

[36] X. Gao, B. Nauta, and E. A. M. Klumperink, "Advantages of Shift Registers Over DLLs for Flexible Low Jitter Multiphase Clock Generation," Circuits and Systems II: Express Briefs, IEEE Transactions on, vol. 55, pp. 244-248, 2008.

[37] E. Klumperink, R. Dutta, R. Zhiyu, B. Nauta, and X. Gao, "Jitter-Power minimization of digital frequency synthesis architectures," in Circuits and Systems (ISCAS), 2011 IEEE International Symposium on, 2011, pp. 165168.

[38] FCC, "Second Report and Order and Memorandum Opinion and Order In the Matter of Unlicensed Operation in the TV Broadcast Bands, Additional Spectrum for Unlicensed Devices Below $900 \mathrm{MHz}$ and in the $3 \mathrm{GHz}$ Band," Nov. 2008. 
[39] FCC, "In the Matter of Unlicensed Operation in the TV Broadcast Bands Additional Spectrum for Unlicensed Devices Below $900 \mathrm{MHz}$ and in the 3GHz Band," Sep. 2010.

[40] S. J. Shellhammer, A. K. Sadek, and W. Zhang, "Technical Challenges for Cognitive Radio in the TV White Space Spectrum," in Information Theory and Applications Workshop, 2009. 


\section{CHAPTER THREE}

\section{Polyphase Multipath Transmitter System Design}

(section 3.1 and some part of section 3.2 of this chapter are taken from the author's paper [1] published in the proceedings of the International Symposium on Circuits and Systems (ISCAS). Some part of Section 3.3, 3.4 and section 3.5 are taken from the author's paper published in the IEEE Journal of Solid State Circuits [2)]].

In chapter 2 some of the flexible transmitter architectures were briefly discussed. Among these was the polyphase multipath upconversion architecture. In this chapter an improvement in the polyphase multipath upconversion is discussed which allows to reduce the number of polyphase paths in comparison to [3], while aiming to suppress ALL the local oscillator (LO) harmonics. The aim is to achieve as high harmonic suppression as possible while keeping the number of paths low. In section 3.1 an analysis of the choices made in the 18-path upconverter are discussed. In section 3.2, the motivation for selecting a particular duty cycle of the LO which led to the choice of an 8-path upconversion is discussed. An analysis discussing the effects of LO duty cycle on the efficiency of the upconverter is presented in section 3.3, while the multiphase LO generation and phase mismatch issues are discussed in section 3.4 and section 3.5 respectively.

\subsection{Poly-phase Multipath System Analysis}

As discussed in section 2.2.1 and in [3], some of the up-converted terms in the implementation of the polyphase multipath up-converter are not cancelled. Taking $\omega_{B B}$ as the input baseband signal and $\omega_{\llcorner O}$ as the LO signal, the most dominant of these uncancelled terms in the output spectrum occurs at $3 \omega_{\llcorner O}+3 \omega_{\mathrm{BB}}$ as discussed in [3] and shown in Figure 3.1 for an 18-path Upconversion. The $3 \omega_{\mathrm{BB}}$ term (in the $3 \omega_{\mathrm{LO}}+3 \omega_{\mathrm{BB}}$ upconverted spectral component) is the $3^{\text {rd }}$ order distortion of the baseband signal, and can be reduced by making the baseband section sufficiently linear or by using digital pre-distortion [4].

If differential baseband and differential LO signals are used and initially assuming that the component at $3 \omega_{\mathrm{LO}}+3 \omega_{\mathrm{BB}}{ }^{1}$ is not dominant, then the first dominant un-cancelled harmonic in an $\mathrm{N}$ path PU occurs at $\mathrm{N}-1[3]$ times the LO frequency. The magnitude of this harmonic

\footnotetext{
${ }^{1}$ This term can also be reduced by making the duty cycle of the $\mathrm{LO}=1 / 3$.
} 
decreases as $\mathrm{N}$ increases, as shown in Figure 3.2. Even with $\mathrm{N}=30$ the harmonic suppression does not reach $-30 \mathrm{dBc}$, while many parallel paths are needed. In the next section we explore options to achieve $<-40 \mathrm{dBc}$ harmonic suppression by a limited number of paths in combination with simple RC filtering. This is relevant to reduce complexity, as an N-path polyphase Transmitter also requires $\mathrm{N}$ Digital to Analog functions in parallel and $\mathrm{N}$ reconstruction filter functions. Also the mixers require multiphase clocks and multiphase analog baseband signals to drive them. Multiphase LO-generation becomes more challenging as the number of paths increase, and will require a higher frequency if dividers are used for flexible multiphase LO-generation.

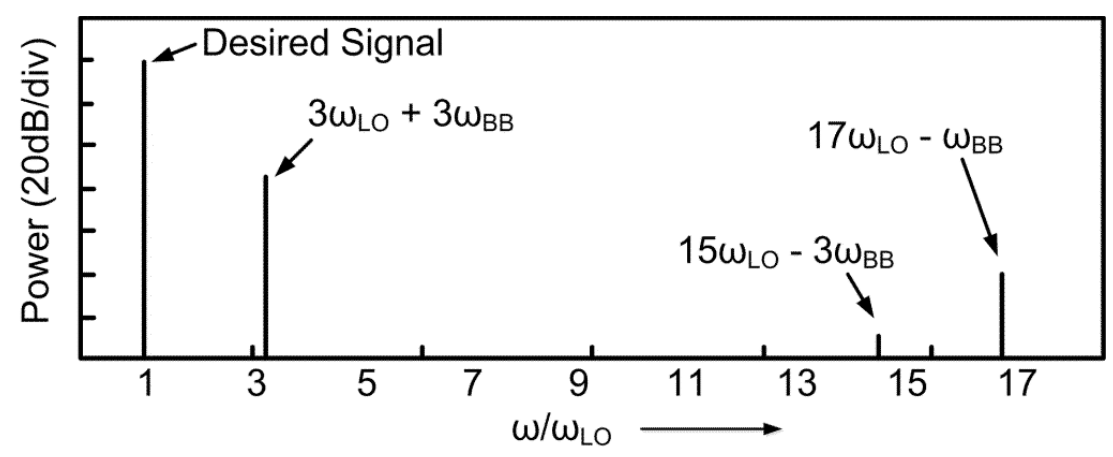

Figure 3.1.Output Spectrum for an 18-path Upconversion of a single tone[3]

Apart from the LO-phase, duty cycle is another degree of design freedom for LOgeneration. In [3] the duty cycle (D) of the LO was made $1 / 3$ to suppress the $3^{\text {rd }}$ LO harmonic. This was done to reduce the $3 \omega_{\mathrm{LO}}+3 \omega_{\mathrm{BB}}$ term which is not cancelled by the multipath architecture, as equation (2.5) in chapter 2 is satisfied for $m=3$ and $j=0$. The magnitude of the $3 \omega_{\mathrm{LO}}+3 \omega_{\mathrm{BB}}$ term relative to the fundamental can be calculated by the relation $\left(A_{p}^{2} \times a_{3} / 3 \times 4 a_{1}\right)$ [5], assuming $D=1 / 2$, where $a_{3}$ and $a_{1}$ are the coefficients of the nonlinearity as described by a Taylor approximation [6] and $A_{p}$ is the amplitude of the input sinusoid. Assuming $\mathrm{A}_{\mathrm{p}}$ to be around $200 \mathrm{mV}, \mathrm{a}_{3} / \mathrm{a}_{1} \approx 3 \mathrm{~V}^{-2}$, this term can be around $-40 \mathrm{dBc}$ for a switched transconductor PU as described in [3]. If some source degeneration is applied for linearization or some RC-filtering is added at the output, this would make this term even lower. So making $D=1 / 3$ is not essential in achieving $-40 \mathrm{dBc}$ suppression of the $3 \omega_{\mathrm{LO}}+3 \omega_{\mathrm{BB}}$ term. Due to mismatches and phase inaccuracies, achieving much better harmonic rejection than $-40 \mathrm{dBc}$ is difficult [3]. Moreover a filter is still needed to reject the first non-cancelled harmonic, which is discussed in the next subsection. 


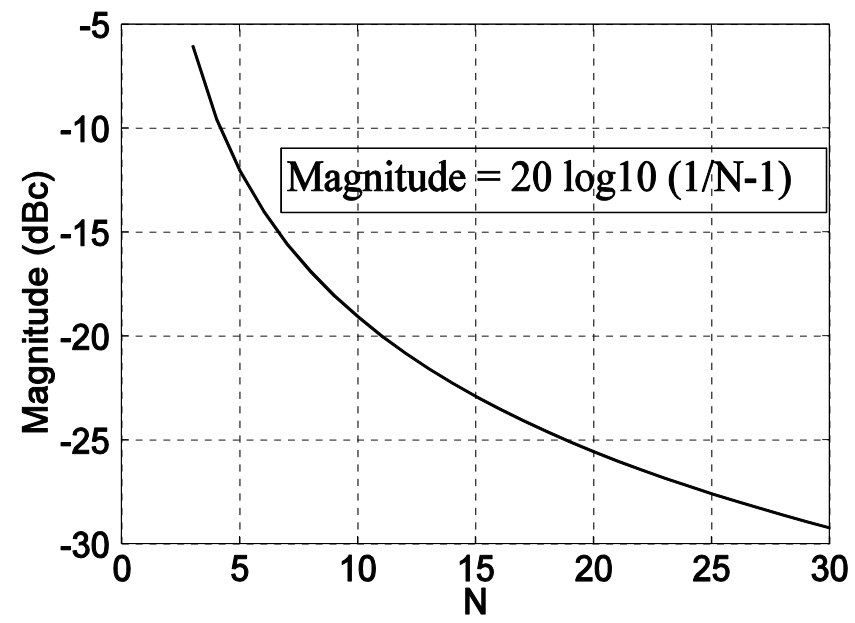

Figure 3.2. Magnitude of the $\mathrm{N}-1^{\text {th }}$ Harmonic in an N path Transmitter driven by poly-phase $50 \%$ duty cycle ideal square-wave LO-signals [1].

\subsubsection{Multipath System with a First order RC filter at the output.}

It would be interesting to see how much suppression can be achieved if a first order tunable $\mathrm{RC}$ low pass filter is placed at the output of a multipath upconverter. This is interesting because in some cases the inherent parasitic capacitances at the output of the upconverter in combination with the antenna load impedance can already provide a first order roll off. Also it is relatively simple to make a bank of switchable capacitors on chip (to achieve tunable first order filtering). The total suppression of the LO harmonics in such a case would be a combination of the suppression due to the multipath system and the RC filter. Assuming that the $-3 \mathrm{~dB}$ corner frequency of such an $\mathrm{RC}$ filter is placed such that the signal loss for the desired $1^{\text {st }}$ harmonic is $\leq 1 \mathrm{~dB}$, then the magnitude of the filter response as a function of the first uncancelled harmonic $\left(\mathrm{N}-1^{\text {th }}\right)$ in an N-path upconversion is shown in Figure 3.3. The roll-off of Figure 3.2 expressed in terms of the $\mathrm{N}-1^{\text {th }}$ harmonic (where $\mathrm{N}$ is variable) is also shown. In essence some signal power would be lost for the sake of simpler filtering and a large tuning range (cut-off frequency inversely proportional to $\mathrm{C}$ ). The signal loss can be avoided by the method to be discussed in section 3.2.1 (Case 2).

Adding the suppression, we can achieve $<-40 \mathrm{dBc}$ combining multipath upconversion and simple RC filtering if $\mathrm{a} \geq 15^{\text {th }}$ harmonic is the first un-cancelled harmonic, implying $\mathrm{N} \geq 16$. So if only a first order filter is used at the output of a multipath system, then sixteen or more paths would be needed to suppress the harmonics to more than $40 \mathrm{~dB}$. This is the case when the duty cycle $\mathrm{D}=1 / 2$. 


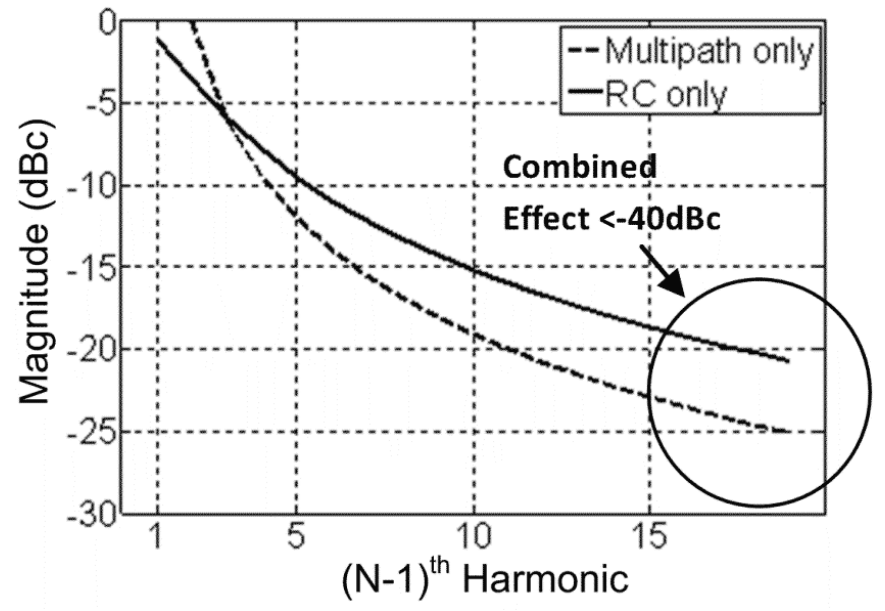

Figure 3.3 Suppression due to 1 st order RC at the $(N-1)^{\text {th }}$ harmonic( $N$ is variable), suppression due to the polyphase multipath upconversion with $50 \%$ duty cycle ideal square-wave also shown [1].

Since the duty cycle of the local oscillator (LO) is a degree of freedom in the harmonic suppression, in the next section we see the benefit of varying the LO duty cycle, to achieve harmonic suppression with less number of paths.

\subsection{Eight Path Transmitter}

It is possible to achieve harmonic suppression by varying the duty cycle D of the LO in a multipath architecture. If we keep $\mathrm{N}$ even, a 180 degrees shifted version of the LO phase is always available (e.g. for $\mathrm{N}=4$, phase $=0^{\circ}, 90^{\circ}, 180^{\circ}, 270^{\circ}$ ), allowing for the suppression of all the even order harmonics. This is desired as the $2^{\text {nd }}$ harmonic becomes dominant when $\mathrm{D} \neq 1 / 2$.

It is preferred to keep $\mathrm{N}$ as low as possible for complexity and power efficiency reasons, while aiming for high harmonic suppression ( 40 to $50 \mathrm{dBc}$ as discussed in Section 2.3). In a 4-path architecture, also referred to as an IQ or image reject mixer, the first few uncancelled harmonics occur around the $3^{\text {rd }}, 5^{\text {th }}, 7^{\text {th }}$ harmonic of the LO whereas in a 6-path design they occur around the $5^{\text {th }}, 7^{\text {th }}, 11^{\text {th }}$ harmonic of the LO (see equation $(2.5)$ in chapter 2)). The magnitude of these harmonics is much stronger than $-40 \mathrm{dBc}$ as seen in Figure 3.2, and requires significant filtering.

An 8-path design [1] presents the opportunity to suppress the two dominant harmonics $\left(7^{\text {th }}\right.$ and $\left.9^{\text {th }}\right)$ simultaneously, while allowing the next two harmonics $\left(15^{\text {th }}\right.$ and $\left.17^{\text {th }}\right)$ to be distant enough for simple $1^{\text {st }}$ order RC filtering. This can be done by varying the duty cycle $\mathrm{D}$ of the LO. The strength of the $7^{\text {th }}$ and $9^{\text {th }}$ LO harmonics for $\mathrm{D}=1 / 2$ is $-16.9 \mathrm{dBc}$ and $-19 \mathrm{dBc}$ respectively. This can be calculated by taking the magnitude of the Fourier series 
coefficients $\left(a_{n}\right.$ and $\left.b_{n}\right)$ of the LO waveform shown in Figure 3.4a.These coefficients and the amplitude of the $\mathrm{n}^{\text {th }}$ harmonic $\mathrm{V}_{\mathrm{n}}$ are:

$$
\begin{aligned}
& a_{n}=\frac{2}{T} \cdot \int_{0}^{T} x(t) \cos (n \omega t) d t=\frac{A}{n \cdot \pi} \sin (2 \cdot \pi \cdot n \cdot D) \\
& b_{n}=\frac{2}{T} \cdot \int_{0}^{T} x(t) \sin (n \omega t) d t=\frac{A}{n \cdot \pi}(\cos (2 \cdot \pi \cdot n \cdot D)-1) \\
& V_{n}=\sqrt{a_{n}^{2}+b_{n}^{2}}=\frac{A}{n \cdot \pi} \sqrt{2-2 \cos (2 \cdot \pi \cdot n \cdot D)}
\end{aligned}
$$

Equation (3.3) is plotted as a function of D in Figure 3.4b, where the desired signal is normalized to $0 \mathrm{dBc}$ for $\mathrm{D}=0.5$. In the figure it can be seen that both the $7^{\text {th }}$ and $9^{\text {th }} \mathrm{LO}$ harmonic are at a level of around $-31 \mathrm{dBc}$ when $\mathrm{D}$ is either $\sim 7 / 16$ or $\sim 9 / 16$. These are the only two points in the Figure $3.4 \mathrm{~b}$ where both the $7^{\text {th }}$ and $9^{\text {th }}$ harmonic components are below $-31 \mathrm{dBc}$ simultaneously [1]. Evaluating equation (3.3) in Table 3.1 we find an optimum duty-cycle of 0.4365 (43.65\%) to achieve simultaneous rejection for both $\mathrm{V}_{7}$ and $\mathrm{V}_{9}$ to $-31.9 \mathrm{dBc}$ compared to the fundamental at $43.65 \%$ duty cycle. The conversion gain for the fundamental is $-4.1 \mathrm{~dB}$, only slightly worse than the $-3.9 \mathrm{~dB}(2 / \pi)$ for $50 \%$ dutycycle. The optimum is close to $\mathrm{D}=7 / 16(43.75 \%)$ [1], where we find $-30.9 \mathrm{dBc}$ and $-33 \mathrm{dBc}$ for the $7^{\text {th }}$ and $9^{\text {th }}$ harmonic. The reason for specifying the LO duty cycle D in a fractional form is related to the way it is often generated using digital dividers as discussed in section 3.4. Comparing the results, we see that the optimum is rather sensitive: a duty-cycle change of $0.1 \%$ from the optimum renders $1 \mathrm{~dB}$ worse rejection. The variation in the $9^{\text {th }}$ harmonic is less of an issue as higher harmonics will undergo more attenuation by low-pass filtering. In section 5.3 on duty cycle control, the circuit design used to keep variations in duty cycle $<$ $0.1 \%$ is discussed.

Table 3.1 shows an improvement in harmonic suppression of at least $13 \mathrm{~dB}$ as compared to the case when $\mathrm{D}=1 / 2$. As the $7^{\text {th }}$ harmonic is more than 2 octaves from the fundamental, a simple first order filter can implement the missing $10 \mathrm{~dB}$ to achieve $<-40 \mathrm{dBc}$ ( 2 octaves $\Leftrightarrow$ $2 \times 6 \mathrm{~dB}=12 \mathrm{~dB}$ filtering). The next two most dominant harmonics for an 8-path transmitter occur at 15 and 17 times the LO, which are at a level of around $-24 \mathrm{dBc}$. A first order filter can have around $20 \mathrm{~dB}$ suppression there, which again brings the result at $<-40 \mathrm{dBc}$. Higher harmonics are at an even lower amplitude level. One might wonder what happens for fewer paths. A 4-path design is the minimum even-N design with image rejection, required for single sideband transmission. In order to allow all the harmonics to be $<-40 \mathrm{dBc}$, the $3^{\text {rd }}$, $5^{\text {th }} \ldots 13^{\text {th }}$ harmonic would need to be significantly suppressed simultaneously, which is not possible with any single $\mathrm{D}$ value. 


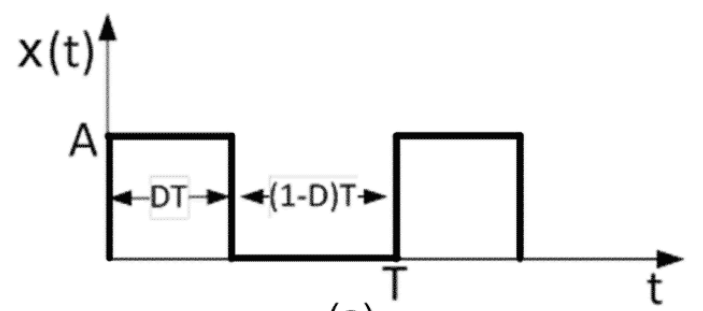

(a)

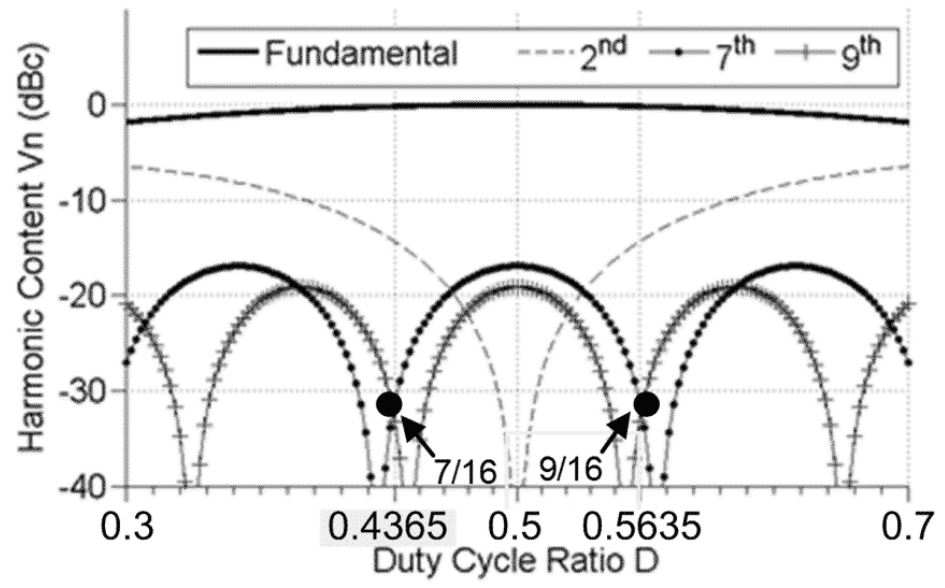

(b)

Figure 3.4 (a) Square wave with duty cycle D (b) LO Harmonic content as a function of $D$.

Table 3.1 Harmonic Strength versus duty cycle

\begin{tabular}{|l|l|l|l|l|}
\hline Duty Cycle & $\mathrm{V}_{1}(\mathrm{~dB})$ & $\mathrm{V}_{2}(\mathrm{~dB})$ & $\mathrm{V}_{7}(\mathrm{~dB})$ & $\mathrm{V}_{9}(\mathrm{~dB})$ \\
\hline $50.00 \%$ & -3.922 & -121.466 & -20.824 & -23.007 \\
\hline $43.55 \%$ & -4.101 & -18.027 & -37.200 & -35.040 \\
\hline $43.60 \%$ & -4.099 & -18.091 & -36.600 & -35.530 \\
\hline $43.65 \%$ & -4.096 & -18.155 & -36.039 & -36.050 \\
\hline $43.70 \%$ & -4.093 & -18.220 & -35.514 & -36.606 \\
\hline $43.75 \%(7 / 16)$ & -4.090 & -18.286 & -35.02 & -37.202 \\
\hline $43.80 \%$ & -4.088 & -18.352 & -34.553 & -37.843 \\
\hline
\end{tabular}




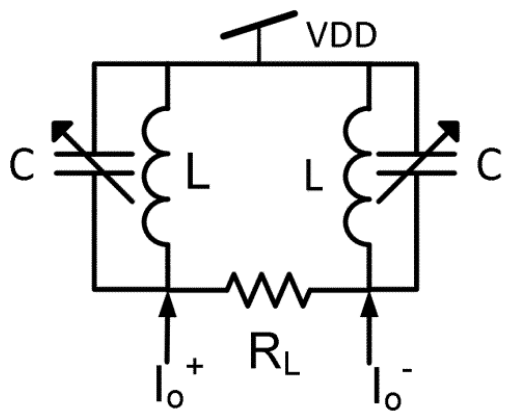

(a)

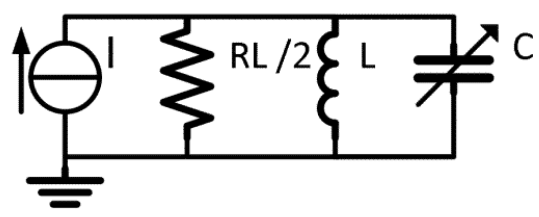

(b)

Figure 3.5 (a) Output Network of multipath PU. (b) Single ended BPF circuit driven by a current source.

A similar argument can be given for not choosing 6 paths. Therefore the next even $\mathrm{N}$ of 8 is chosen.

\subsubsection{Eight path Transmitter and tunable RF Filter}

The output network of a multipath Upconverter can be implemented as shown in Figure 3.5a, where $\mathrm{I}_{\mathrm{o}}$ represents the mixer output current and $\mathrm{R}_{\mathrm{L}}$ the (antenna) load impedance. The presence of an inductor [3] allows the output node to be biased at VDD and therefore allows for high output voltage swings around VDD, which is desired for high output power. If the capacitors values $\mathrm{C}$ are tunable and the inductor value $\mathrm{L}$ is kept fixed, it is possible to realize a tunable band-pass filter (BPF) response.

For simplicity, consider the single ended (half-circuit) version of the output network driven by a current source as shown in Figure 3.5b. The Q of the parallel RLC network is approximately given by:

$$
Q=R_{L} \sqrt{\frac{C}{L}}
$$

For low $\mathrm{Q}$ value $(<<1)$, the inductor value is $\operatorname{big}\left(\mathrm{L}>>\mathrm{R}^{2} \mathrm{C}\right)$, and for a high $\mathrm{Q}(\geq 1)$, the inductor value is small $\left(\mathrm{L} \leq \mathrm{R}^{2} \mathrm{C}\right)$.

For briefness and convenience, in this section big $\mathrm{L}$ and small $\mathrm{L}$ refer to this range of $\mathrm{L}$ values. A big L results in broadband behavior, and a small $\mathrm{L}$ in narrowband BPF behavior around the LC resonance frequency (see Figure 3.6). 


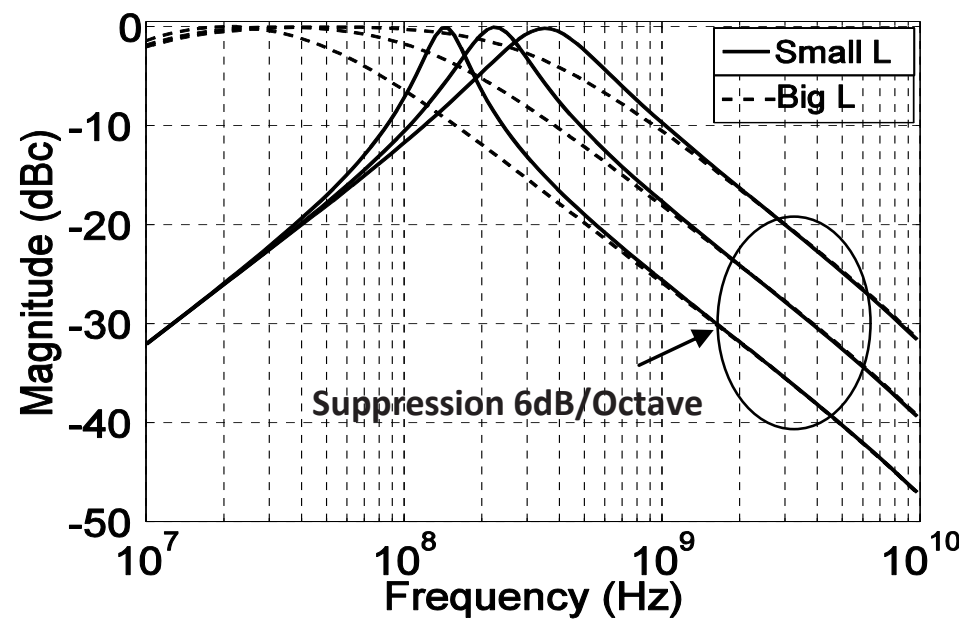

Figure 3.6 Tunable BPF response [1].

Above resonance, $\mathrm{RC}$ filtering dominates ultimately resulting in a roll-off of $6 \mathrm{~dB} /$ octave as shown in Figure 3.6. Closer to resonance, the roll-off can be either lower (Big L, low-Q case) or higher (Small L case with high-Q peaking behavior). This in part also depends on how the inductors are implemented due to losses in the coils. Certainly at low frequencies below $1 \mathrm{GHz}$, on-chip coils are big and have rather low Q. It is typically preferred to use off-chip high-Q coils. In this section we consider two cases, the first case with two big inductors L, often referred to as "RF chokes" resulting in a low Q RLC network, the other with two smaller inductors allowing for peaking in the filter response resulting in a high $\mathrm{Q}$ RLC network.

\section{Case 1: Low $Q$ RLC network (big $L$ )}

When the inductor value is big, the filtering is mainly due to the RC filter network. The harmonic suppression of such a filter is the same as shown in Figure 3.3. To have the filter cover a wide frequency range for the fundamental the capacitor value needs to be tunable. In order to determine the range of values of the capacitor, we again consider the single ended case as shown in Figure 3.5b. The $\mathrm{C}$ value can be given as:

$\mathrm{C}=\frac{1}{2 \cdot \pi \cdot \frac{R_{L}}{2} \cdot f_{C}}$

where $f_{c}$ is at the second harmonic $\left(2 \times \omega_{\text {LO }}\right)$ to keep the desired signal loss $\leq 1 \mathrm{~dB}$. For a frequency range of $100-800 \mathrm{MHz}$ and assuming (single ended) $\mathrm{R}_{\mathrm{L}} / 2=50 \Omega$, then at the lowest frequency the $\mathrm{C}$ value should be $16 \mathrm{pf}$ and at the higher end it should be $2 \mathrm{pf}$. These values are small enough to be implemented on chip. It can be seen also that the cut off 
frequency is inversely proportional to the $C$ value. The suppression achievable with such an $\mathrm{RC}$ filter is given in Table 3.2 .

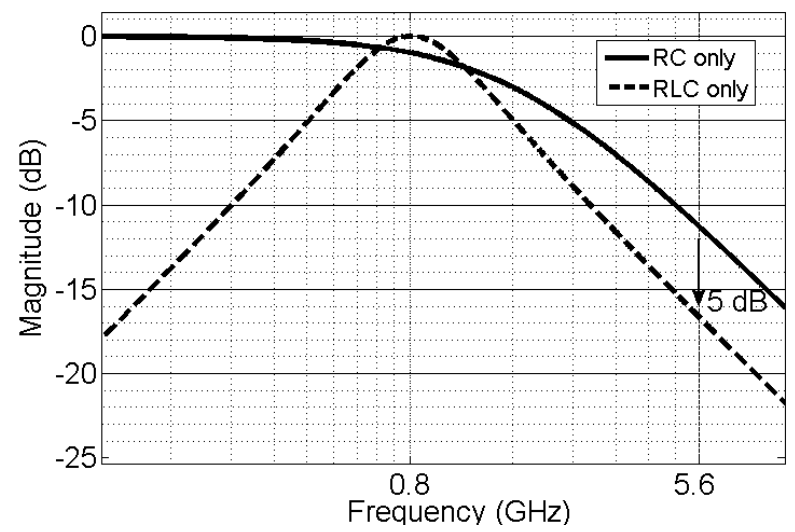

Figure 3.7 Difference in harmonic suppression of RC and RLC filter

\section{Case 2: High Q RLC network (small L)}

In the first case the inductance of the filter did not play an active role in the filter behavior. If more harmonic suppression is required then it can be useful to utilize small inductance value instead of an RF choke. This results in a higher $Q$ value $(\geq 1)$ and can provide at least $6 \mathrm{~dB}$ improvement in the suppression of higher harmonics as compared to the first case. This is because in the low Q RLC (or just RC) case the corner frequency is placed at $2 \times \omega_{\text {LO }}$ to limit signal loss to $1 \mathrm{~dB}$, whereas in the high Q RLC case the desired signal is placed almost at the LO frequency so higher harmonics undergo more attenuation as shown in Figure 3.7. In the figure the desired frequency component at $800 \mathrm{MHz}$ undergoes $1 \mathrm{~dB}$ more attenuation for the $\mathrm{RC}$ case and the undesired $7^{\text {th }}$ harmonic at $5.6 \mathrm{GHz}$ undergoes $5 \mathrm{~dB}$ more suppression for the RLC network case therefore in total $6 \mathrm{~dB}$ better harmonic suppression is achieved for the RLC case. Similar to the first case the inductors are assumed to be off chip and have low loss. The Q of the parallel RLC network is approximately given by:

$$
Q=\frac{R_{L}}{\omega_{0} \cdot L}
$$

The $\mathrm{Q}$ of the filter for a fixed $\mathrm{R}_{\mathrm{L}}$ and $\mathrm{L}$ decreases with higher resonance frequencies $\omega_{0}$. Therefore we define the suppression for the highest frequency covered by the LO, so that for all the lower frequencies the filter would have higher $\mathrm{Q}$ and hence higher suppression. If we assume that at the highest LO frequency the $\mathrm{Q}=1$, then we can place the upconverted 
signal at the resonance frequency of the filter and get roughly $6 \mathrm{~dB} /$ octave suppression of the higher harmonics. Ideally there is no loss of the upconverted signal, which implies better efficiency as compared to the first case. Interestingly each doubling of the $\mathrm{Q}$ also gives roughly $6 \mathrm{~dB}$ of additional suppression as can be observed in Figure 3.8, due to the peaking behavior.

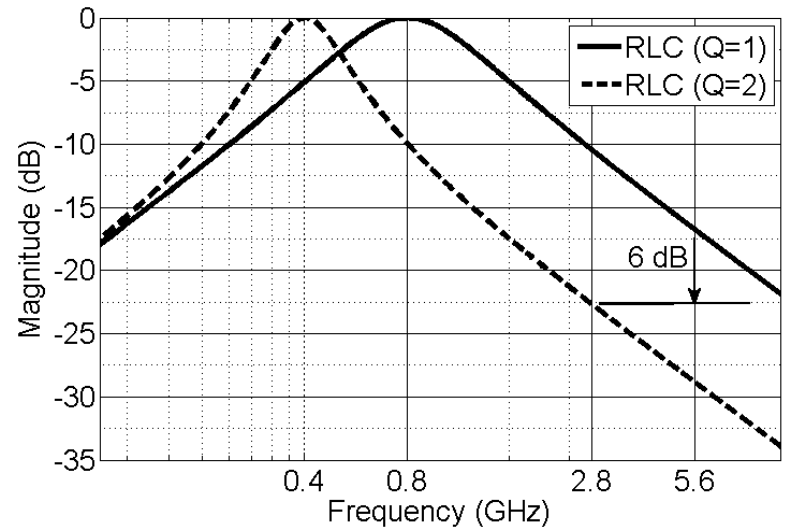

Figure $3.86 \mathrm{~dB}$ higher suppression (shown at the $7^{\text {th }}$ harmonic) with doubling of $Q$, due to halving of $\omega_{0}$ in (3.6).

The (worst-case) suppression at the highest LO-frequency is shown in Figure 3.9 along with the RC filter suppression shown in Figure 3.3. The figure shows the filter suppression at different harmonics of the LO in the output spectrum. It can be seen that the RLC filter has $6 \mathrm{~dB}$ better suppression for higher harmonics as compared to the RC (RLC with $\mathrm{Q}<<1$ ) filter. Using eq. (3.6), the inductor value needed to achieve a $\mathrm{Q}=1$ at $800 \mathrm{MHz}$ is $10 \mathrm{nH}$. The $\mathrm{C}$ value required at this resonance frequency is $3.95 \mathrm{pf}$ and at $100 \mathrm{MHz}$ it is $32 \mathrm{pf}$. An inductor of $10 \mathrm{nH}$ may be small enough to be considered for implementation on chip, but the associated losses will degrade efficiency.

As the antenna is off-chip anyway for these frequencies, off-chip coils are probably preferred. Also, if more power is needed than given the voltage breakdown conditions of a power up-converter (PU) chip, adding a transformer is an option. To summarize, the amplitude of the dominant spectral components in various scenarios of an 8-path transmitter are given in Table 3.2. It is important to note here that the tuning range in case of an RC filter is proportional to $1 / \mathrm{C}$ while in the RLC case it is proportional to $1 / \sqrt{ } \mathrm{C}$. So although the High Q RLC is good for harmonic rejection, its tuning range is less than the $\mathrm{RC}$ (Low Q RLC) filter for a given range of $\mathrm{C}$ values. On the other hand the RC filter gives loss but has better tuning range. So both approaches have their value. 


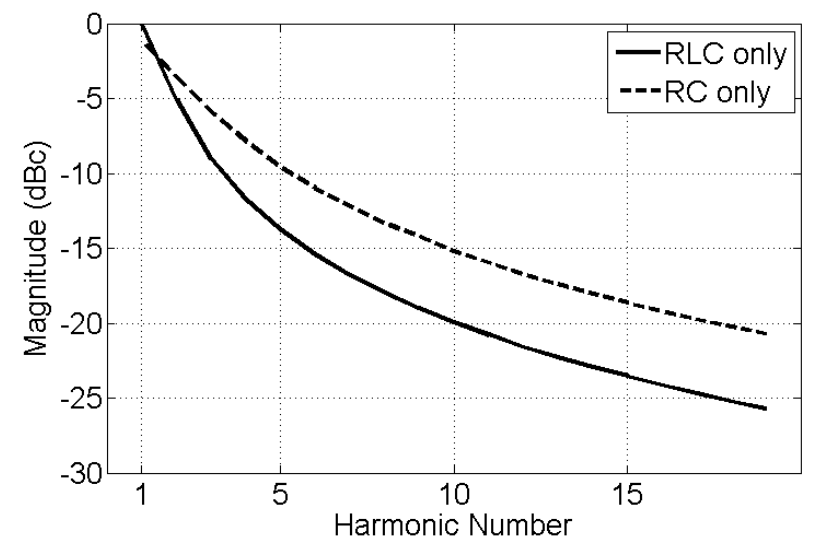

Figure 3.9 Suppression with RLC filter $(Q=1)$ and $1^{\text {st }}$ order RC Filter (corner frequency at $2^{\text {nd }}$ harmonic)

Table 3.2 Harmonic Magnitude in an 8-path Transmitter

\begin{tabular}{|c|c|c|c|c|c|c|}
\hline & $\begin{array}{l}\text { Spectral } \\
\text { components }\end{array}$ & $\begin{array}{c}3 \omega_{\mathrm{LO}}+3 \omega_{\mathrm{BB}} \\
(\mathrm{dBc})\end{array}$ & $\begin{array}{c}7 \omega_{\mathrm{LO}}-\omega_{\mathrm{BB}} \\
(\mathrm{dBc})\end{array}$ & $\begin{array}{c}9 \omega_{\mathrm{LO}}+\omega_{\mathrm{BB}} \\
(\mathrm{dBc})\end{array}$ & $\begin{array}{c}15 \omega_{\mathrm{LO}}-\omega_{\mathrm{BB}} \\
(\mathrm{dBc})\end{array}$ & $\begin{array}{c}17 \omega_{\mathrm{LO}}+\omega_{\mathrm{BB}} \\
(\mathrm{dBc})\end{array}$ \\
\hline 1 & $\begin{array}{l}50 \% \text { Duty } \\
\text { Cycle }\end{array}$ & $<-38$ & -17 & -19 & -24 & -25 \\
\hline 2 & $\begin{array}{l}\sim 7 / 16 \text { Duty } \\
\text { Cycle }\end{array}$ & $<-40$ & -32 & -32 & -24 & -25 \\
\hline 3 & $\begin{array}{l}\text { RC with } \\
f_{c}=2 \times \omega_{\text {LO }}\end{array}$ & -4 & -11 & -13 & -17 & -19 \\
\hline 4 & $\begin{array}{l}\text { RLC with } \\
Q=1\end{array}$ & -9 & -17 & -19 & -23 & 25 \\
\hline 5 & $\begin{array}{l}\text { Total } \\
\text { Suppression } \\
{[2+3]}\end{array}$ & $<-44$ & -43 & -45 & -41 & -44 \\
\hline 6 & $\begin{array}{l}\text { Total } \\
\text { Suppression } \\
{[2+4]}\end{array}$ & $<-49$ & -49 & -51 & -47 & -50 \\
\hline
\end{tabular}




\subsection{Efficiency vs. duty cycle}

We have seen in Section 3.2 that for harmonic suppression a duty cycle of $43.65 \%$ is preferred. As power efficiency is a concern for a Power Upconverter [3], we will in this section analyze the duty cycle ratio which provides the optimal power efficiency. In the analysis we assume that the mixers used for upconversion are switched gm mixers [7], where a transconductor is switched on and off by a switch. We consider the single ended ${ }^{2}$ PU using switched $\mathrm{g}_{\mathrm{m}}{ }^{3}$ multipath mixers directly driving the load $\mathrm{R}_{\mathrm{L}}$ as shown in Figure 3.10 .

The mixer efficiency is defined as:

$$
\eta=\frac{P_{r f-\text { desired }}}{P_{d c}}
$$

Where $\mathrm{P}_{\text {rf-desired }}$ is the desired up-converted signal power delivered to the load and $\mathrm{P}_{\mathrm{dc}}$ is the dc power consumed in the mixer.

For simplicity we first assume ideal and perfectly linear transconductors with a large-signal V-I conversion $(\mathrm{Gm})$ equal to its small-signal $g_{m}$ and ideal switches. The single tone baseband signal applied at the input of the $g_{m}$ blocks can be given as:

$V_{b b_{n}}=V_{g t o}+V_{p} \cos \left(\omega_{b b}+\varphi_{n}\right)$

Where $\mathrm{V}_{\text {gto }}$ is the dc bias voltage (e.g. the overdrive voltage of a MOSFET above threshold $\left(\mathrm{V}_{\text {th }}\right) \cdot \mathrm{V}_{\mathrm{p}}$ is the baseband amplitude voltage, which should not exceed $\mathrm{V}_{\text {gto }}$ to avoid clipping ${ }^{4}$.

The dc power consumed in $\mathrm{N}$ path mixer can be given by:

$P_{d c}=V_{d d} \cdot I_{d c}$

\footnotetext{
${ }^{2}$ Single ended is assumed for simplicity in analysis, in the implemented upconverter (chapter 5) the output power is taken differentially.

${ }^{3}$ It is assumed that no current flows through the $\mathrm{g}_{\mathrm{m}}$ block when the LO switch is off.

${ }^{4}$ Although ideal V-I conversion is assumed but clipping would occur in practical circuits.
} 


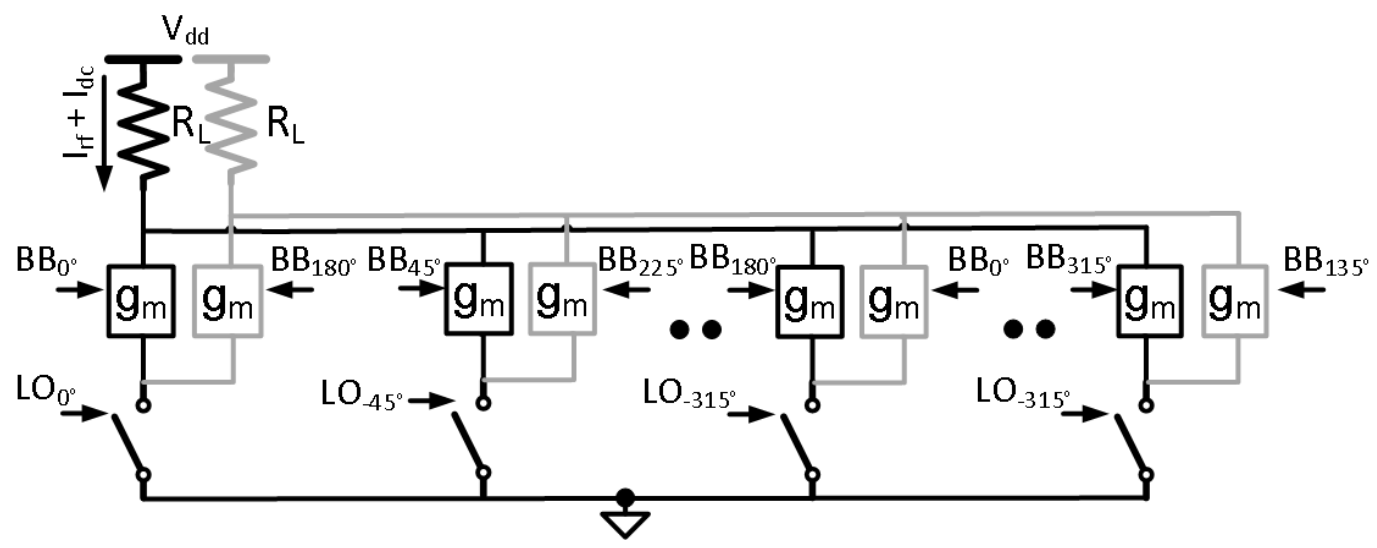

Figure 3.10 Single ended switched $g_{m}$ mixer

The dc current consumed in one mixer (assuming the transconductor is linear) can be given by:

$I_{d c 1}=g_{m 1} \cdot V_{g t o} \cdot D$

$\mathrm{D}$ is the duty cycle during which the mixer remains ON. Since ideally all the mixers have the same dc bias voltage and the same $g_{m}$, the dc current consumed in each of the $\mathrm{N}$ mixers is the same so the total dc current consumption can be given as:

$I_{d c}=N \cdot g_{m 1} \cdot V_{g t o} \cdot D$
$P_{d c}=\cdot V_{d d} \cdot N \cdot g_{m 1} \cdot V_{g t o} \cdot D$

Note that for linear switched transconductor mixers the baseband cosine signal does not affect total dissipation significantly, as the increase of a current in one mixer is accompanied by an equal decrease in another one, with the anti-phase baseband signal.

The up-converted signal power can be expressed as:

$P_{r f-\text { desired }}=\left(\frac{I_{r f-\text { desired }}}{\sqrt{2}}\right)^{2} \cdot R_{L}$

Where the factor $\sqrt{2}$ in the denominator is to convert $I_{\text {rf-desired }}$ into rms value. While the desired up-converted signal current is equal to:

$I_{r f-\text { desired }}(t)=\frac{N \cdot g_{m 1} \cdot V_{p} \cdot V_{1} \cos \left(\omega_{L O}+\omega_{b b}\right) t}{2}$ 


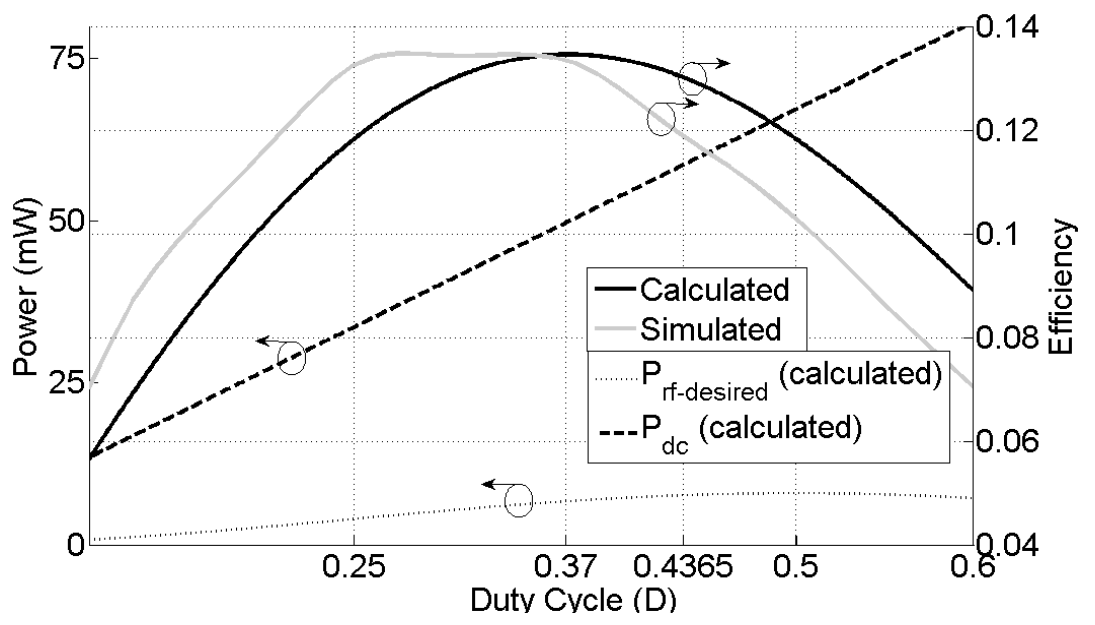

Figure 3.11 Efficiency peaks at 37\% Duty Cycle.

Where the factor 2 in the denominator is due to the product of two cosines ( $b b$ and LO), $\mathrm{V}_{1}$ is the amplitude of the fundamental harmonic of the LO as given in (3.3), for $n=1$ and A replaced by the amplitude of the desired signal current. In all the paths the desired signal has the same phase, therefore they add up constructively, which explains $\mathrm{N}$ in (3.14). Substituting (3.3) in (3.14) and taking magnitude:

$$
\begin{aligned}
& I_{r f \text {-desired }}=\frac{N \cdot g_{m 1} \cdot V_{p} \cdot \sqrt{2-2 \cos (2 \cdot \pi \cdot D)}}{2 \cdot \pi} \\
& \mathrm{P}_{r f-\text { desired }}=\frac{N^{2} \cdot g_{m 1}{ }^{2} \cdot V_{p}{ }^{2} \cdot(2-2 \cos (2 \cdot \pi \cdot D))}{8 \cdot \pi^{2}} \cdot R_{L}
\end{aligned}
$$

Figure 3.11 shows a plot of equation (3.7), (3.12) and (3.16) as a function of D, where the following practical values were used: $\mathrm{N}=8, \mathrm{~g}_{\mathrm{m}}=35 \mathrm{mS}, \mathrm{V}_{\mathrm{p}}=0.2, \mathrm{~V}_{\mathrm{gto}}=0.32, \mathrm{~V}_{\mathrm{dd}}=1.5$. The efficiency curve, peaks at a duty cycle of around 0.37

The desired signal $\left(\mathrm{P}_{\text {rf-desired }}\right)$ starts to decrease after $\mathrm{D}=0.5$, while the $\mathrm{DC}$ power $\left(\mathrm{P}_{\mathrm{dc}}\right)$ continues to increase as shown in Figure 3.11, therefore intuitively the optimal point should lie somewhere before that. In order to assess the validity of this very coarse model, circuit simulations (using the mixers discussed in Section 5.5) in a standard 160nm CMOS process are also shown in Figure 3.11. The predicted shape is roughly right, but with a shift. This difference is mainly due to the assumption that one mixer path does not influence the other and also assuming linear devices. Simulations done with different circuit parameters show the optimum efficiency point lies in the range of $\mathrm{D}=0.25$ and $\mathrm{D}=0.35$. We conclude that the targeted duty-cycle $\mathrm{D}=0.4365$ lies between $\mathrm{D}=0.5$ and the optimum efficiency point. Although not exactly optimal, the power efficiency compared to $\mathrm{D}=0.50$ is $10-20 \%$ better. 


\subsection{Generating LO Duty Cycle}

An 8-path transmitter requires 8-phase LO signals to drive the mixer switches. The LO phases can be generated by a divide-by- 8 circuit [8]. For low phase mismatch it is desired to drive all flip-flops (FF) in a divide-by- 8 circuit by the same master clock. A chain of $8 \mathrm{FF}$ cells is needed to generate all the phases. It is possible to make the duty cycle ratio $\mathrm{k} / 8$ (where $\mathrm{k}=1,2 \ldots 7$ ), by using digital dividers [8], therefore a nice fraction of the duty cycle is desired. However to make the duty cycle ratio $7 / 16$ requires the implementation of 8 additional FF cells (for divide by 16). This is possible but requires extra power consumption and also limits the maximum output frequency in a particular process technology, since the input frequency of the divider chain then has to be 16 times higher than the LO frequency. Moreover, from Table 3.1 it is seen that it is actually better for the $7^{\text {th }}$ harmonic rejection to tune the duty-cycle to a slightly lower value than $7 / 16$.

Since 0.4365 is rather close to 0.50 , we use an 8 -phase $50 \%$ duty-cycle clock generated by a divide-by-4 circuit and delay the rising edge (only by a ratio of 0.063 ) to reduce dutycycle (see Figure 3.12). This low delay introduces a negligible phase noise degradation compared to other sources of noise. A buffer afterwards can again make the edge steep. To ensure a clean output spectrum, the harmonic content can be monitored and controlled by adapting the duty-cycle. A cognitive radio transceiver would likely have a spectrum analyzer on board [9-12]. The harmonics of the transmit frequency can be detected ${ }^{5}$ by the spectrum analyzer provided they fall within the band of the analyzer, which is likely for low transmit-frequencies, where the high harmonics are the most problematic. If the $7^{\text {th }}$ or $9^{\text {th }}$ harmonics are at a very high frequency outside the input frequency range of the spectrum analyzer, detection will require additional techniques.

Figure 3.12 shows that starting from a duty cycle ratio of $1 / 2$ the rising edge can be slowed to reach the required ratio of $\sim 7 / 16$. This way the effective LO waveform has un-symmetric rise and fall times but the effective time the mixer switch remains ON can be decreased, thus approximating a 7/16 ratio. If the LO waveform switches between 0 and VDD and the mixer switch turns on at $\mathrm{VDD} / 2$, the required edge delay as a ratio of the LO period is $1 / 8$. For a $1 \mathrm{GHz} \mathrm{LO}$, this means a rise time of $125 \mathrm{ps}$.For a frequency agile transmitter making the falling edge delayed by a ratio of $1 / 8$ of the LO period over a wide

\footnotetext{
${ }^{5}$ Spectrum sensing receivers should be very sensitive and able to measure very weak signal levels and with good degree of accuracy. Even if currently such receivers are not available commercially, we think that this will change. See for instance the performance numbers achieved in [9-12]. In this thesis we assume that detection of problematic harmonic content is feasible and aim for duty-cycle tuning to control this problem.
} 


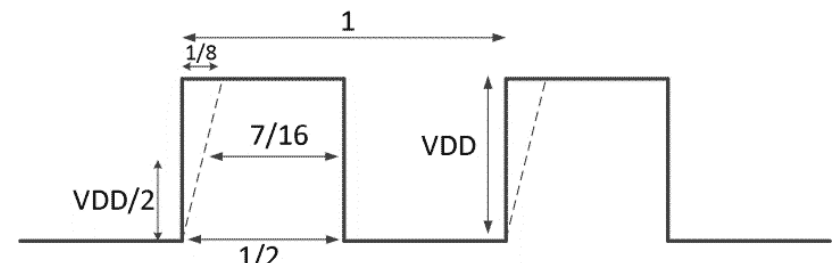

Figure 3.12 Making $D=7 / 16$ from $D=1 / 2$

frequency range requires adding a tuning mechanism to the LO waveform. Such a tuning mechanism will be discussed in Chapter 5 on duty cycle control.

\subsection{Effect of Phase Mismatch}

A mathematical analysis of the effect of mismatch on multi-path polyphase circuits can be found in [13], providing an estimate of the Harmonic Rejection Ratio of cancelled $(C)$ harmonics as:

$$
E\left(H R R_{k, m}\right)_{C}=\frac{P_{k, m, \text { reference }}}{P_{k, m, \text { rejected }}}=\frac{N^{2}}{(N-1)\left(\frac{\sigma_{\varepsilon}{ }^{2}}{a_{1}^{2}}+k_{L O}{ }^{2} \sigma_{\theta}{ }^{2}+m^{2} \sigma_{\delta}{ }^{2}\right)}
$$

where $E\left(H R R_{k, m}\right)$ is the expected value for a tone resulting from the $\mathrm{k}^{\text {th }}$ harmonic of the LO and $\mathrm{m}^{\text {th }}$ harmonic of the baseband signal. $\mathrm{P}_{\mathrm{k}, \mathrm{m} \text {,reference }}$ is the power before rejection, produced by a single path transmitter, while $\mathrm{P}_{\mathrm{k}, \mathrm{m} \text {,rejected }}$ is the power after (imperfect) rejection by the multipath technique due to the presence of mismatch. $\sigma_{\theta}{ }^{2}, \sigma_{\delta}{ }^{2}, \sigma_{\varepsilon}{ }^{2}$ are the variances of the stochastic variables $\theta$ (LO phase mismatch error), $\delta$ (BB phase mismatch error) and $\varepsilon$ (amplitude mismatch error), respectively. Note that a higher number of paths has a positive effect on HRR. In order to find the effect of mismatch on the harmonics which are not cancelled (index NC), a similar analysis is done, resulting in [2]:

$E\left(H R R_{k, m}\right)_{N C}=\frac{P_{k, m, \text { reference }}}{P_{k, m, \text { non-rejected }}}=1-\frac{(N-1)}{N^{2}} \cdot\left(\frac{\sigma_{\varepsilon}^{2}}{a_{1}^{2}}+k_{L O}{ }^{2} \sigma_{\theta}{ }^{2}+m^{2} \sigma_{\delta}{ }^{2}\right)$

Note that the expected value of this equation for small values of amplitude and phase mismatch is very close to one. This is because the non-cancelled harmonics even in the presence of mismatch almost fully add up in phase. In the measurement section in Chapter 5 we will observe this effect. 


\subsection{Conclusion}

The analysis of a previous implementation of the polyphase multipath technique shows that making the duty cycle of the LO $1 / 3$ is not essential in achieving $\geq 40 \mathrm{~dB}$ harmonic suppression. Therefore the duty cycle of the LO could be made different than $1 / 3$ to suppress other dominant harmonics. It is shown that a lot of parallel paths are needed to achieve high harmonic suppression. If the duty cycle is kept at $1 / 2$, then $\geq 16$ paths are needed, to achieve $40 \mathrm{~dB}$ harmonic suppression in combination with a first order RF filter. Making a 16 path polyphase upconverter can be complex and will consume a lot of power. Therefore some other design option is required.

An 8-path polyphase transmitter has the two most dominant spectral products around the $7^{\text {th }}$ and $9^{\text {th }}$ harmonic of the LO. It is shown that an 8-path polyphase transmitter with a duty cycle of $43.65 \%$ can suppress both the $7^{\text {th }}$ and $9^{\text {th }}$ harmonic of the LO by more than $31 \mathrm{~dB}$ compared to the fundamental signal. If a $1^{\text {st }}$ order RF filter is applied the overall rejection becomes more than $40 \mathrm{~dB}$ for all the harmonics. This can be improved by $6 \mathrm{~dB}$ if a high $\mathrm{Q}$ tunable RLC network is used at the output but at the cost of capacitor range. It could be possible to achieve the required duty cycle by delaying the rising edge of the LO starting from a duty cycle of $50 \%$ and reaching $\sim 43.65 \%$. The duty cycle ratio of $43.65 \%$ is better than $50 \%$ in terms of power efficiency, while the non-cancelled harmonics even in the presence of phase mismatch almost fully add up in phase.

\section{References}

[1] S. Subhan, E. A. M. Klumperink, and B. Nauta, "Towards suppression of all harmonics in a polyphase multipath transmitter," in Circuits and Systems (ISCAS), 2011 IEEE International Symposium on, 2011, pp. 2185-2188.

[2] S. Subhan, E. A. M. Klumperink, A. Ghaffari, G. J. M. Wienk, and B. Nauta, "A 100-800 MHz 8-Path Polyphase Transmitter With Mixer Duty-Cycle Control Achieving < $-40 \mathrm{dBc}$ for ALL Harmonics," Solid-State Circuits, IEEE Journal of, vol. 49, pp. 595-607, Mar. 2014.

[3] R. Shrestha, E. A. M. Klumperink, E. Mensink, G. J. M. Wienk, and B. Nauta, "A Polyphase Multipath Technique for Software-Defined Radio Transmitters," Solid-State Circuits, IEEE Journal of, vol. 41, pp. 2681-2692, 2006.

[4] Y. Xi, D. Chaillot, P. Roblin, L. Wan-Rone, L. Jongsoo, P. Hyo-Dal, et al., "Poly-Harmonic Modeling and Predistortion Linearization for SoftwareDefined Radio Upconverters," Microwave Theory and Techniques, IEEE Transactions on, vol. 58, pp. 2125-2133, 2010. 
[5] B. Razavi, RF Microelectronics. Upper Saddle River, NJ: Prentice Hall, 1998.

[6] E. Mensink, E. A. M. Klumperink, and B. Nauta, "Distortion cancellation by polyphase multipath circuits," IEEE Trans. Circuits Syst. II, Exp. Briefs, vol. 57, pp. 1785-1794, Sep. 2005.

[7] E. A. M. Klumperink, S. M. Louwsma, G. J. M. Wienk, and B. Nauta, "A CMOS switched transconductor mixer," Solid-State Circuits, IEEE Journal of, vol. 39, pp. 1231-1240, 2004.

[8] Z. Ru, N. A. Moseley, E. Klumperink, and B. Nauta, "Digitally Enhanced Software-Defined Radio Receiver Robust to Out-of-Band Interference," Solid-State Circuits, IEEE Journal of, vol. 44, pp. 3359-3375, 2009.

[9] M. S. Oude Alink, E. A. M. Klumperink, A. B. J. Kokkeler, Z. Ru, W. Cheng, and B. Nauta, "Using Crosscorrelation to Mitigate Analog/RF Impairments for Integrated Spectrum Analyzers," Microwave Theory and Techniques, IEEE Transactions on, vol. PP, pp. 1-11, 2013.

[10] M. S. Oude Alink, E. A. M. Klumperink, A. B. J. Kokkeler, M. C. M. Soer, G. J. M. Smit, and B. Nauta, "A CMOS-Compatible Spectrum Analyzer for Cognitive Radio Exploiting Crosscorrelation to Improve Linearity and Noise Performance," Circuits and Systems I: Regular Papers, IEEE Transactions on, vol. 59, pp. 479-492, 2012.

[11] M. S. Oude Alink, E. A. M. Klumperink, M. C. M. Soer, A. B. J. Kokkeler, and B. Nauta, "A 50Mhz-To-1.5Ghz Cross-Correlation CMOS Spectrum Analyzer for Cognitive Radio with 89dB SFDR in 1Mhz RBW," in New Frontiers in Dynamic Spectrum, 2010 IEEE Symposium on, 2010, pp. 1-6.

[12] M. S. Oude Alink, A. B. J. Kokkeler, E. A. M. Klumperink, G. J. M. Smit, and B. Nauta, "Fast, Sensitive, Efficient, and High-SFDR Spectrum Sensing with Crosscorrelation Energy Detection," Journal on Emerging and Selected Topics on Circuits \& Systems (JETCAS), on:"Advanced Circuits and Systems for CR/SDR Applicaions", Dec. 2013.

[13] E. Mensink, E. A. M. Klumperink, and B. Nauta, "Distortion cancellation via polyphase multipath circuits," in Circuits and Systems, 2004. ISCAS '04. Proceedings of the 2004 International Symposium on, 2004, pp. I-1098101 Vol.1. 


\section{CHAPTER FOUR}

\section{Mixed-Signal System Design}

This chapter deals with the mixed-signal multiphase baseband signal generation of the 8path up-converter presented in the previous chapter. The aim is to derive a set of requirements and present feasible design directions. As discussed previously, the aim of the complete transmitter is to have the local oscillator (LO) originated harmonics better than $40 \mathrm{dBc}$ in the output spectrum. We aim to keep the harmonics originating from the baseband paths much better than $-40 \mathrm{dBc}$ and even aim for lower so that they do not become a bottleneck.

The 8-path polyphase transmitter requires eight baseband paths to drive the 8-path mixers. This could be realized in the digital domain by the weighted addition of two quadrature baseband signals. The method to generate these multiphase baseband signals is presented in section 4.1. The baseband signals require a certain DAC resolution to achieve $<-50 \mathrm{dBc}$ unwanted signal components. The resolution requirements of the Digital-to-Analog Converter (DAC) are discussed in section 4.2.

A reconstruction filter is conventionally required after the DAC, to remove unwanted DAC images and noise. In literature, multiple parallel DACs whose outputs are combined have been shown to be beneficial in terms of DAC image cancellation. Techniques exploiting multiple parallel DACs to cancel or suppress some of the DAC images in a multipath up converter are discussed in section 4.3.

The DAC design should be such that the mismatch between the multiphase baseband paths can be minimized. A possible solution is to share the voltage reference of a ladder DAC or another possibility is to use one time-interleaved DAC. In section 4.4 these DACs are discussed.

A technique to relax reconstruction filtering requirements is to use oversampling of the digital data. The extra cost is area and power consumed in the digital domain. It is shown that using an interpolation filter for oversampling is feasible and does not dominate the overall power budget in the given technology node. The digital interpolation filter design used for oversampling is presented in section 4.5. 


\subsection{Multiphase Baseband Generation}

Before proceeding to discuss multiphase baseband generation, it is instructive to see the characteristics of an ideal phase shifter and how it relates to an ideal time delay, since these terms will be used in the text. As can be seen in Figure 4.1, the ideal phase shifter has a constant phase over frequency while the ideal delay has a linear phase over frequency [1]. This behavior can only be approximated in practical circuits.

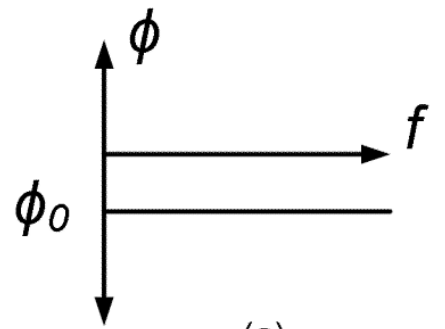

(a)

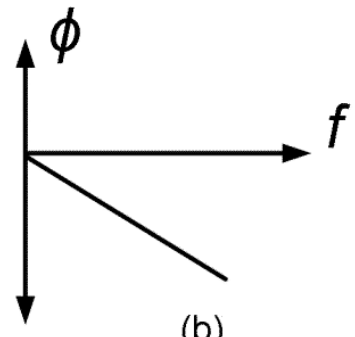

(b)

Figure 4.1. Ideal phase shifter (a) ideal time delay (b) (frequency and phase axis are linear)[1]

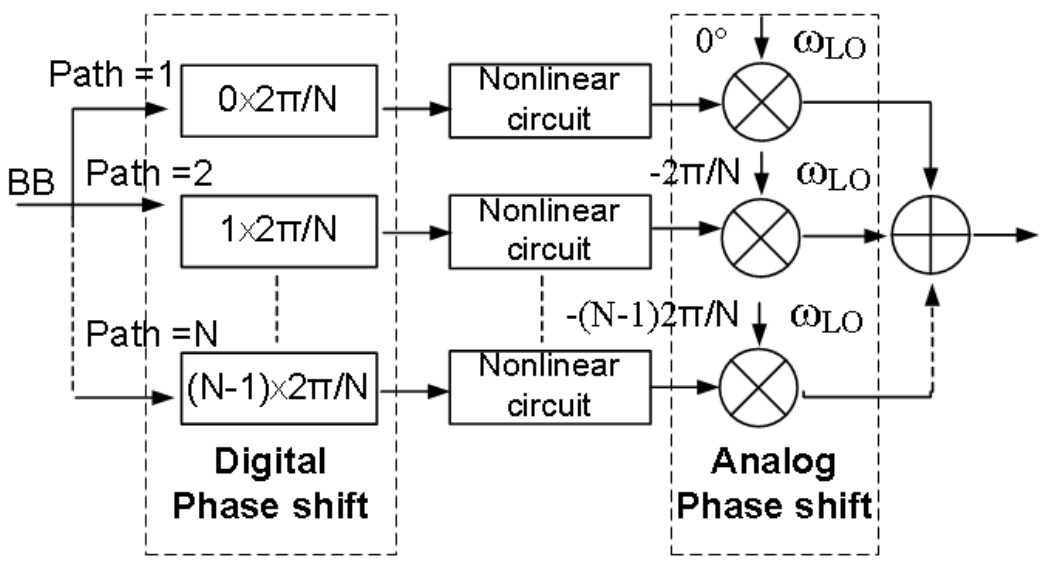

Figure 4.2. First set of phase shifts in digital.

One of the reasons why the poly-phase multipath technique is particularly suitable for a transmitter is the possibility to exploit digital techniques in baseband to implement the first set of phase shifts shown in Figure 4.2(see chapter 2/3). With the advancement in IC technology more complex digital circuits can be made to approximate ideally wanted behavior. The second set of phase shifters are implemented via (digital) LO-phase shifting 
in analog mixers. In contrast, in a receiver the RF input would need to be phase shifted in the analog domain which can be a challenge for wide fractional bandwidths[2].

In order to understand how the multiphase digital baseband signals can be generated, it is instructive to understand how the multiphase transmitter relates to a conventional fully analog single sideband transmitter[3]. For a Cognitive radio transmitter, spectral efficiency is vital and it is obviously preferred to not transmit the same (redundant) information in both the upper and lower sideband. Image rejection allows for realizing a single sideband transmitter. We will analyze the image cancellation properties of the mixers here using cosine and sine functions following [2],[3], and verify results by simulation using the same signals.

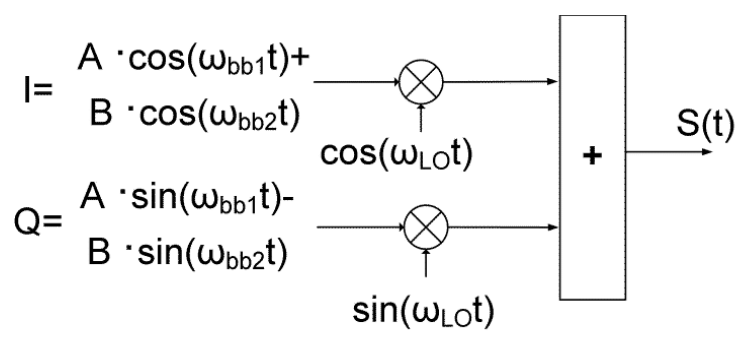

(a)
$B \cdot \cos \left(\omega_{L O}+\omega_{B B 2}\right) t$

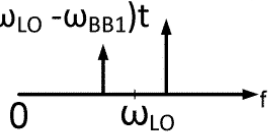

(b)

Figure 4.3(a) Conventional Single sideband upconversion. (b) Up-converted spectrum occurs around the $\omega_{\text {Lo }}$ frequency.

Figure 4.3a shows the conventional single sideband transmitter architecture[3] and Figure $4.3 \mathrm{~b}$ the resulting upconverted spectrum around the LO. The input cosine term is referred to as I, while the input sine term is referred to as the $\mathrm{Q}$ signal. In mathematics:

$$
\begin{aligned}
& I=A \cdot \cos \left(\omega_{b b 1} t\right)+B \cdot \cos \left(\omega_{b b 2} t\right) \\
& Q=A \cdot \sin \left(\omega_{b b 1} t\right)-B \cdot \sin \left(\omega_{b b 2} t\right) \\
& s(t)=I \cdot \cos \left(\omega_{L O} t\right)+Q \cdot \sin \left(\omega_{L O} t\right) \\
& s(t)=A \cdot \cos \left(\omega_{L O} t-\omega_{b b 1} t\right)+B \cdot \cos \left(\omega_{L O} t+\omega_{b b 2} t\right)
\end{aligned}
$$

Here the baseband signals with a phase difference of $-90^{\circ}$ among them appear on the high side of the LO after upconversion while the baseband signals with a phase difference of 
$+90^{\circ}$ among them appear on the low side of the LO after upconversion as seen from (4.1),(4.2) and (4.4).

Often the A and B signals are implemented differentially (signals with $180^{\circ}$ difference between them as shown in Figure 4.4) to achieve even order distortion suppression. In essence there are then 4 single ended baseband signals, or equivalently 2 differential baseband signals as shown in Figure 4.4. It should be noted that A and B are part of one baseband signal while after upconversion with an LO frequency their images (either upper or lower sideband) are ideally cancelled after the summation. Ideally the image frequency components as well as the LO signal are cancelled but in practice mismatches will lead to incomplete cancellation. Note however that both the residual image and LO-signal fall inside the transmitted signal band and do not interfere with other users of the spectrum, which are out of that band.

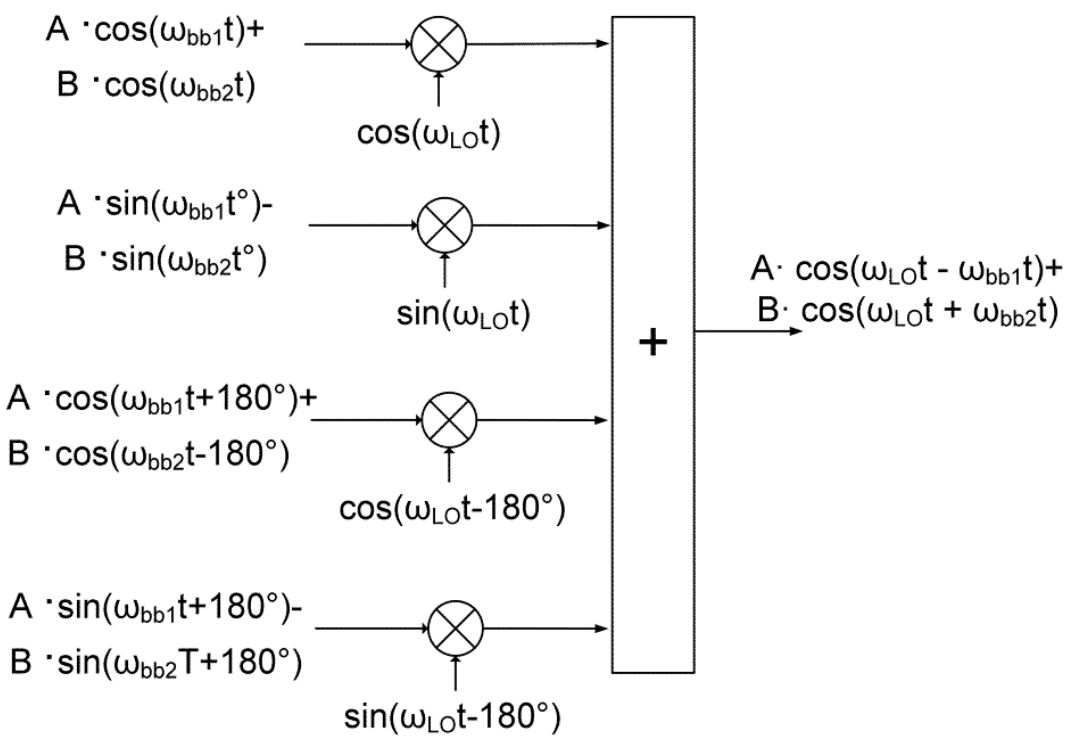

Figure 4.4. Upconversion with two differential baseband signals to cancel even order distortion.

For an 8-path software defined radio/ cognitive radio transmitter, digital streams corresponding to the A and B signals are needed. Not only differential A and B baseband streams are required but also additional baseband phases. In order to generate the single sideband up-converted spectrum as shown in Figure 4.3b, for an 8-path transmitter the architecture of Figure 4.5 can be used. Four additional baseband phases are required compared to the conventional upconverter in Figure 4.4. To generate the additional phase shifted baseband streams, a weighted addition of the input A and B streams is used. This 
can be accomplished in the digital domain via a vector modulator [4] architecture as shown in Figure 4.6. The figure shows how to generate the 45 degree phase but the same principle can be used to generate the 135 degree phase. It can be seen that the required phases can be generated from the commonly used I/Q baseband data and without requiring additional baseband inputs.

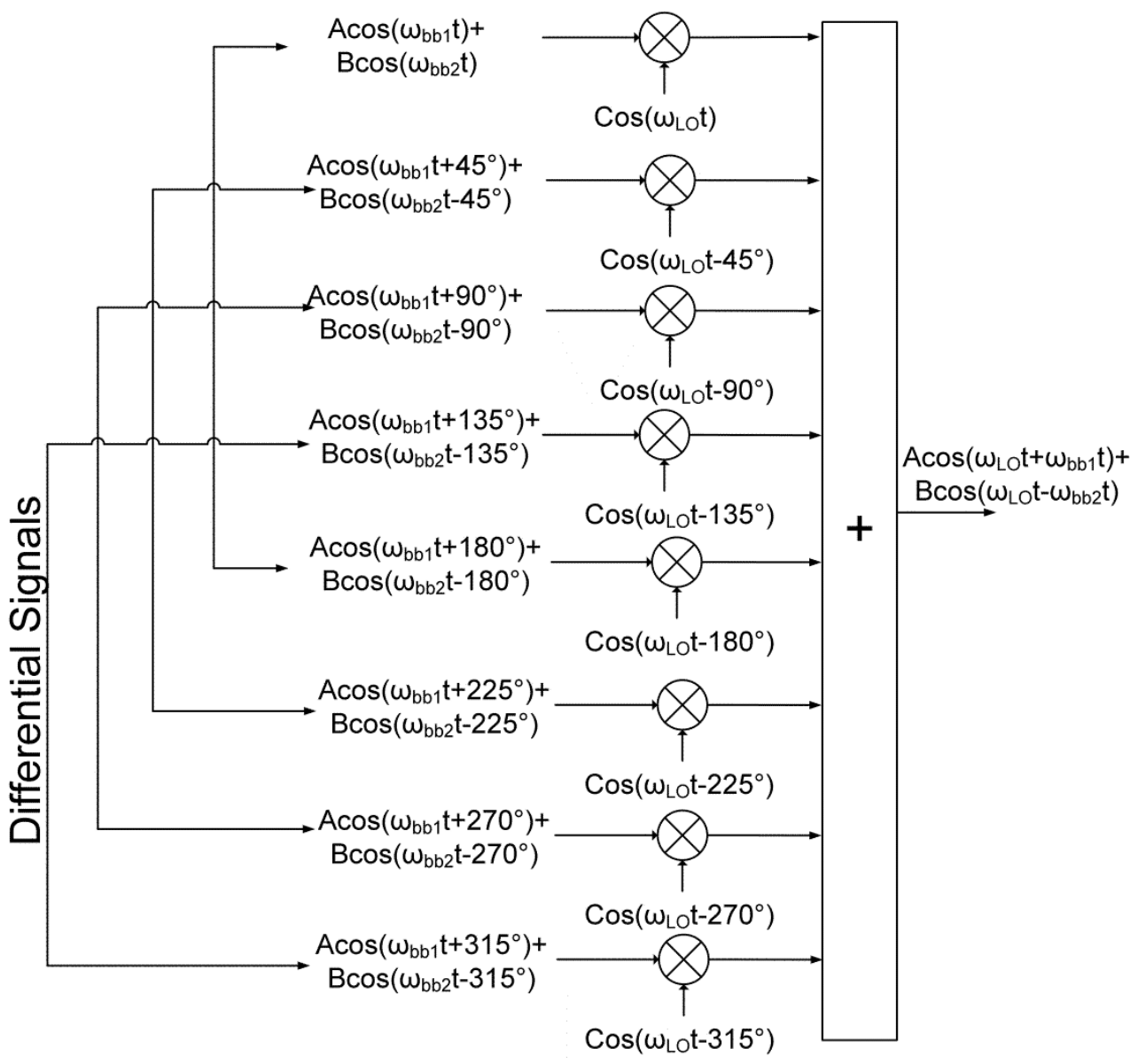

Figure 4.5.Multiphase baseband required for 8-path upconversion. 


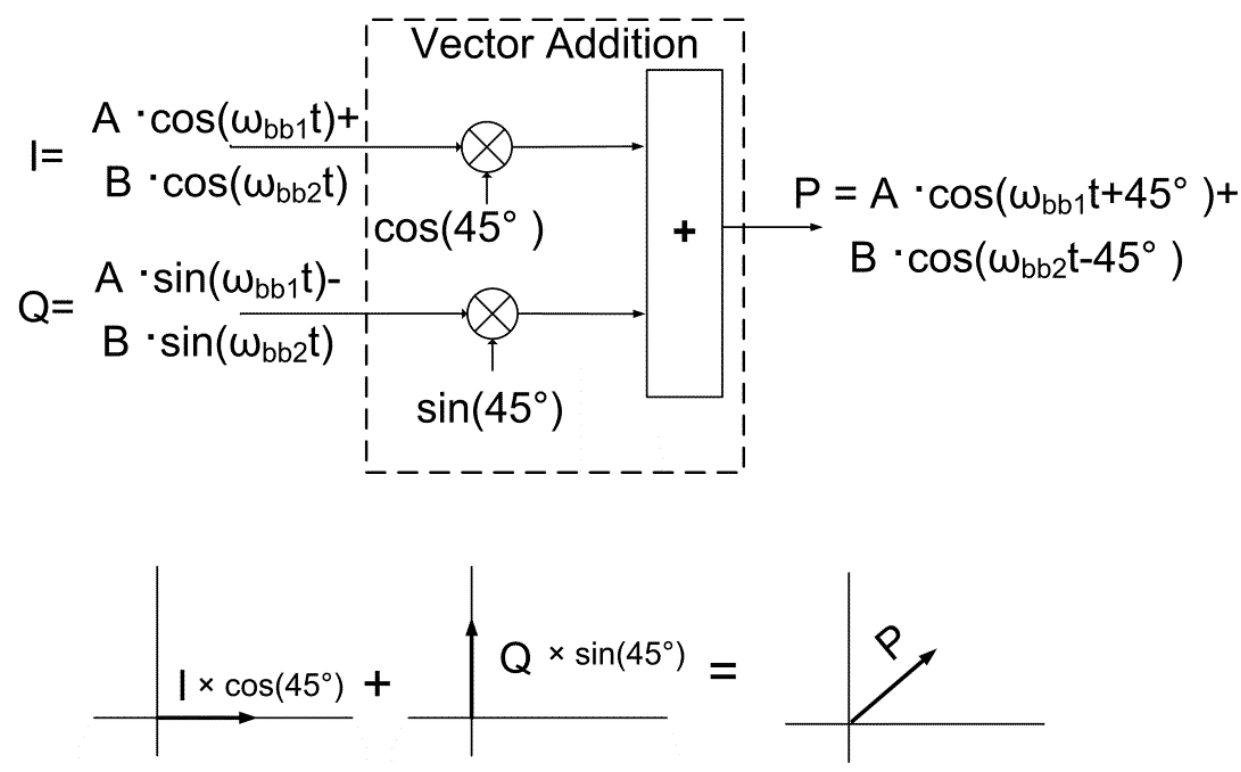

Figure 4.6. Fixed weighted vector addition to generate phase shifted baseband (only one of the 8-generated phases is shown).

Note that only fixed coefficients $(1 / \sqrt{ } 2)$ are required to generate the phase shifts halfway between the I/Q signals. Two multiplications and one addition would be needed to implement the vector modulator. If differential DAC's are used then the 180 degrees shifted version of the baseband signals can be generated also in the analog domain. Overall then two additional differential DAC's would be required as compared to a conventional IQ transmitter.

A digital phase shifter architecture was synthesized in Cadence synthesis tool to generate the 8 digital phase shifted baseband signals based on the vector modulator architecture shown in Figure 4.6. The VHDL code of the design can be found in the APPENDIX B. In $160 \mathrm{~nm}$ CMOS, it would consume an area of $\left(0.01 \mathrm{~mm}^{2}\right)$. It has 16 -bit processing resolution while having 8, 8-bit outputs (digital resolution will be discussed in section 4.2), running at $50 \mathrm{MHz}$, and expected (pre-layout) power consumption of only $2.5 \mathrm{~mW}$. Compared to the overall transmitter power and area (see benchmark Table in Chapter 5) of larger than $120 \mathrm{~mW}$ and area of $0.1 \mathrm{~mm}^{2}$, this phase shifter architecture consumes much less power and area. 


\subsection{DAC Resolution Impact on a Poly-phase Multipath Transmitter}

In the previous chapter it was shown that most of the harmonics produced due to nonlinearity in the PU design can be cancelled using the multipath principle. These harmonics were cancelled on the assumption that analog signals are driving the mixers. When we consider the digital baseband signals and their conversion to the analog domain, quantization errors occurs which tend to produce harmonics of the baseband signal [5]. It should be noted that as mentioned in [6], some authors refer to the quantization errors as noise, however quantization errors show "in a limited sense noise properties" [6]. These harmonics of the baseband signal would dominate the output spectrum for low resolution digital to analog conversion in the absence of reconstruction filter. Possibly the multipath technique might be an option to suppress these terms so that synergy between DAC and upconverter design occurs. Several questions may arise, e.g.:

- What would happen if digital signals are converted to an amplitude discrete representation in the presence of the multipath technique?

- For both single tone or multi-tone sinusoids, what effect does finite D/A resolution have on the output of a multipath transmitter?

These questions are investigated first with the assumption that both sets of phase shifts shown in Figure 4.7 are ideal (section 4.2.1). Then the scenario where the second set of phase shifts are provided with mixers (having frequency translation) is investigated in section 4.2.2.

\subsubsection{Multipath Technique applied to a Uniform DAC with ideal Phase Shifter}

If a pure sinusoid is amplitude quantized in an ADC (resembling a stair-case), then uniform quantization results in pure harmonic distortion [7], and the output spectrum shows distortion terms (when sampled, these distortion components will alias, if their frequencies are higher than Nyquist [7]) which are multiples of the fundamental frequency, especially for low resolution [6]. The output spectrum of a Digital to Analog conversion with a digital sine input with the same resolution would show the same distortion in the absence of reconstruction filtering as in both cases the time domain signal is the same stair-case approximation of a sine. 


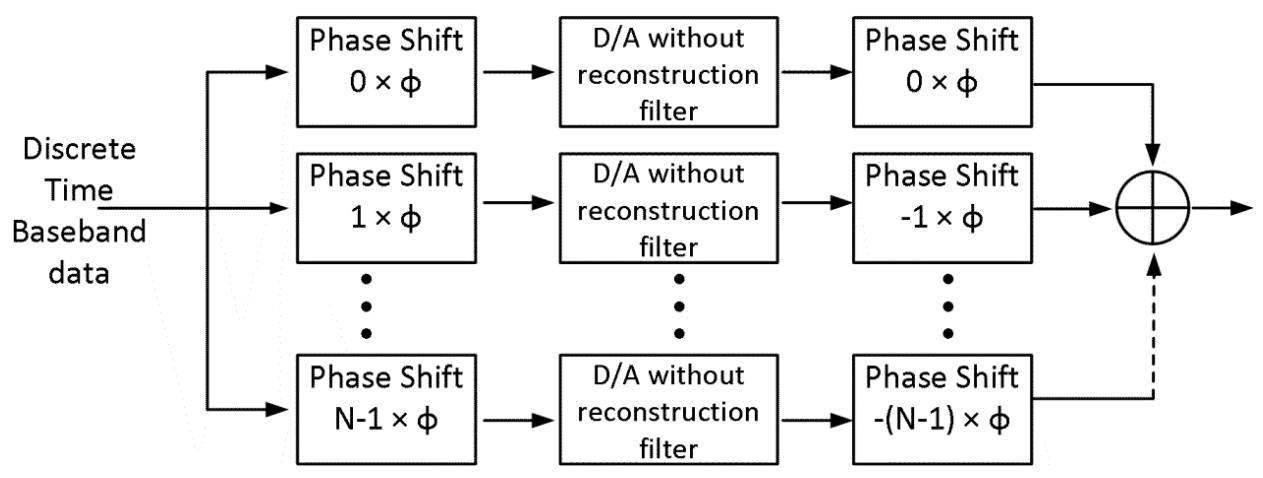

Figure 4.7. Multipath Technique applied to an uniform DAC without reconstruction filter.

This effect of uniform quantization on an input signal such as

$$
x(t)=A(t) \cdot \sin ((2 \cdot \pi \cdot f+\emptyset) \cdot t)
$$

is shown in [5],[6] to produce the following output :

$$
y=\sum_{p=1}^{\infty} A_{p} \sin (p \cdot(2 \cdot \pi \cdot f+\emptyset) \cdot t)
$$

where $A_{p}$ is the amplitude of the $p^{\text {th }}$ harmonic of the quantized signal. As seen in (4.6), the phase of the $p^{\text {th }}$ harmonic is also $p$ times the input phase. This characteristic of the phase is essential for the principle operation of multipath technique. Recalling the modeling of nonlinearity in a multipath system from equation (2.1), the phase of the $\mathrm{k}^{\text {th }}$ harmonic at the output, rotates by $\mathrm{k}$ times the input phase. Note that the uniform quantizer output in (4.6) and the output of a nonlinear system described by (2.1) show a similar kind of distortion. Hence the multipath technique applied to a uniform quantizer, when the input is sinusoid, might be able to cancel out harmonics produced at the output. We will examine this further below.

In order to verify the effect of multipath on such a distortion, the model of Figure 4.7 is used. For simulation purposes the baseband data is assumed to be a discrete time representation of a single sinusoid. This is because distortion due to amplitude quantization would be easier to visualize for a sinusoid as compared to multiple tones.

The D/A conversion block in Figure 4.7 only serves to discretize the amplitude of the input sinusoid. As only the effect of amplitude discretization of input baseband data using the multipath is investigated and not the time discretization effects, therefore the input 
baseband data in Figure 4.7 is assumed to be highly oversampled. Hence in essence it mimics almost a continuous time signal. Phase shifts shown in Figure 4.7 are assumed to be ideal.

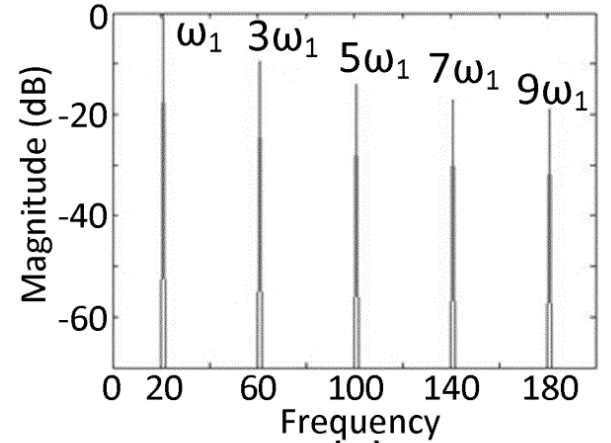

(a)

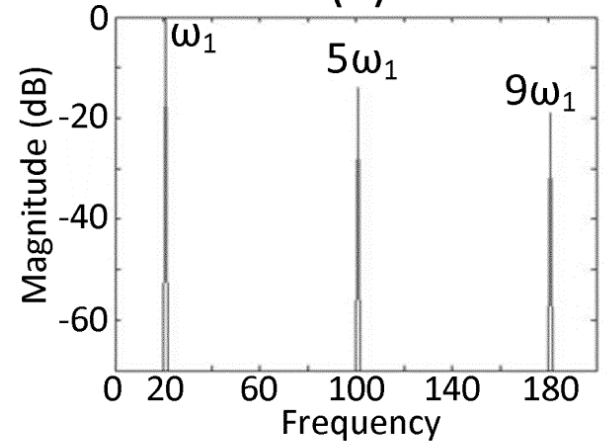

(b)

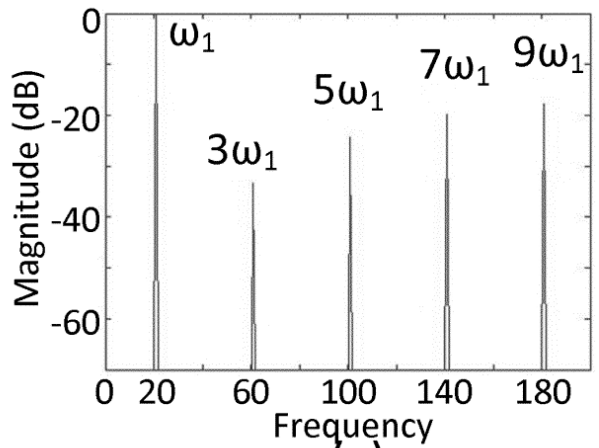

(c)

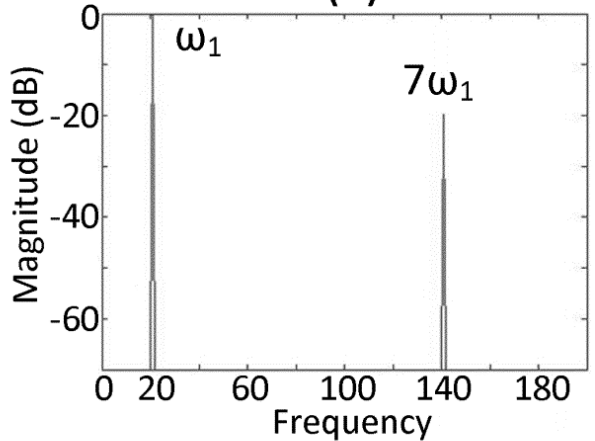

(d)

Figure 4.8 Output Spectrum of the model in Figure 4.7 for a single tone input and (a) 1 bit quantization and one path (b) 1 bit quantization and 4 paths (c) 2 bit quantization and 1 path (d) 2 bit quantization and 6 paths.

Figure 4.8 shows Matlab simulation results for the output spectrum of Figure 4.7 for single tone input data. First as a test case the input signal was coarsely discretized to only two levels, i.e. 1 bit quantization. Figure 4.8(a) and (b) show the simulation results of applying one and then four paths to a 1 bit quantized sinusoid. The output of one path shows only odd harmonic terms $\left(\omega_{1}, 3 \omega_{1}, 5 \omega_{1} \ldots\right)$ as the time domain signal is fully symmetric if mirrored in the time axis (assuming no dc-offsets). For four paths the 3rd harmonic is cancelled and the first non-cancelled harmonic is the fifth, consistent with theory (see section II in [8]). To show that this cancellation also works for a different number of paths and quantization levels, Figure 4.8(c) shows the spectrum of 2 bit quantized sinusoid after 1 path and Figure 4.8(d) for 6 paths. It can be seen that in this case the first non-cancelled harmonic is the seventh, corresponding to the theory in [8]. Simulations for different 
number of paths and various quantization levels confirm that the first non-cancelled harmonic of the quantization process is the $(n+1)^{\text {th }}$ where $n$ is the number of paths.
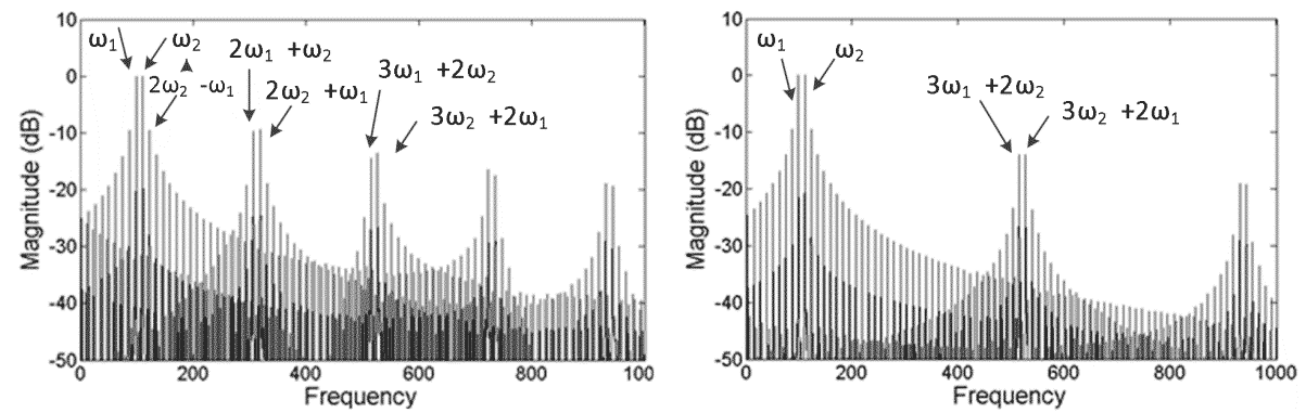

Figure 4.9. Output Spectrum of two tone applied to the model in Figure 4.7 (a) 1 bit quantized one path system (b) 1 bit quantized and 4 path system.

Simulations with two-tone inputs were also done to check correspondence with the theory in [8]. For tones at $\omega_{1}$ and $\omega_{2}$, intermodulation products occurring at $2 \omega_{2}-\omega_{1}, 2 \omega_{1}-\omega_{2}, 3 \omega_{2}$ $2 \omega_{1}, 3 \omega_{1}-2 \omega_{2}, \ldots$ are expected to remain uncancelled, and so are adjacent intermodulation terms (separated by $\omega_{1}-\omega_{2}$ ) around $i \cdot N+1$. Figure 4.9(a) shows the spectrum of a 2-tone signal applied to a 1 bit quantizer for 1 path and Figure 4.9(b) exploiting 4 paths. From the figure it can be seen that with 4 paths, all terms are cancelled except for terms satisfying eq. 4 in [8]: $p+q=j x n+1$, where $p$ and $q$ are integer values. For $n=4$, the terms $3 \omega_{1}+2 \omega_{2}$ and $3 \omega_{2}+2 \omega_{1}$ satisfy this condition $(3+2=1 \times 4+1)$, and also its adjacent intermodulation terms, which remain un-cancelled. Increasing the number of quantization levels or dithering [9] can reduce the highest spur level and make the spectrum of the quantized signal more clean.

From simulation results of single and two tone inputs it can be concluded that the multipath technique is able to cancel many of the harmonics of a uniform quantizer in line with the theory in [8] (section II). However since some significant terms remain, its usefulness in achieving a cleaner output spectrum with limited DAC resolution is limited. 


\subsubsection{Multipath Technique applied to a Uniform DAC with Mixer as Phase Shifter}

In previous subsection it was assumed that the phase shifters were ideal. In fact in the multipath Power up-converter (PU) design [8] the second set of phase shifts are implemented using mixers, as they provide a phase shift. However, they also have nonlinearity. So the question arises what happens after the summation in Figure 4.7, in the presence of the PU nonlinearity (PU shown in Figure 2.10) and the distortion caused by amplitude quantization of input data in the $\mathrm{D} / \mathrm{A}$ block.

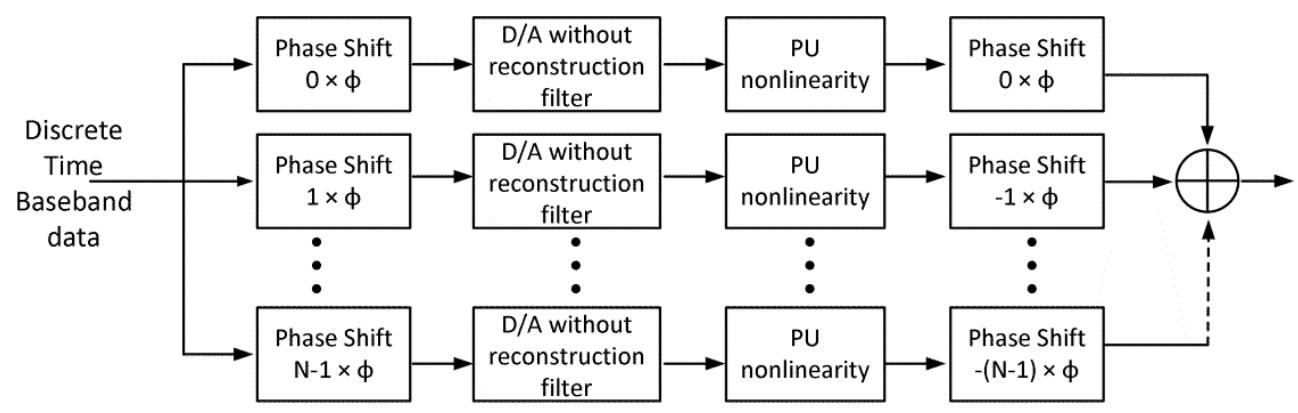

Figure 4.10. Power Upconverter (PU) nonlinearity added to the model of Figure 4.7

The model shown in Figure 4.10 was simulated in Matlab (code in Appendix A), where the $\mathrm{PU}$ nonlinearity is modeled by a power series expansion, the mixer is modeled as an ideal switch, while the first set of phase shifters is again assumed to be ideal. The D/A block is modeled as a uniform quantizer as in the previous subsection without any reconstruction filtering. The output is made differential to cancel the even order terms.

Since the switching mixer produces both sum and difference frequencies a lot of harmonics are produced, as shown in Figure 4.11, for a six path system with three bits quantization. Figure 4.11(a) is for single tone input and Figure 4.11(b) for 2 tone inputs which explains the dense nature of the second graph. The figure shows the desired up-converted signal is at $\omega_{\mathrm{LO}}+\omega_{\mathrm{bb}}$ and the first un-cancelled harmonic of the PU nonlinearity is at $5 \omega_{\mathrm{LO}}-\omega_{\mathrm{bb}}$.

According to [2] for a 6 path system the first un-cancelled harmonic of the PU should occur at $5 \omega_{\mathrm{LO}}-\omega_{\mathrm{bb}}$, so this fits to theory. Simulations carried out for various quantization levels and number of paths showed that the non- cancelled harmonics of the PU non-linearity remain in accordance with [8]. The analysis to determine which distortion terms, produced only due to amplitude quantization, are cancelled and which remain un-cancelled after the multipath becomes complex because of the added non-linearity of the PU. Instead of 
determining which harmonics of the quantization process get cancelled another criterion, SFDR (discussed in next paragraph) is used to judge the effect of multipath on the upconverted output spectrum.
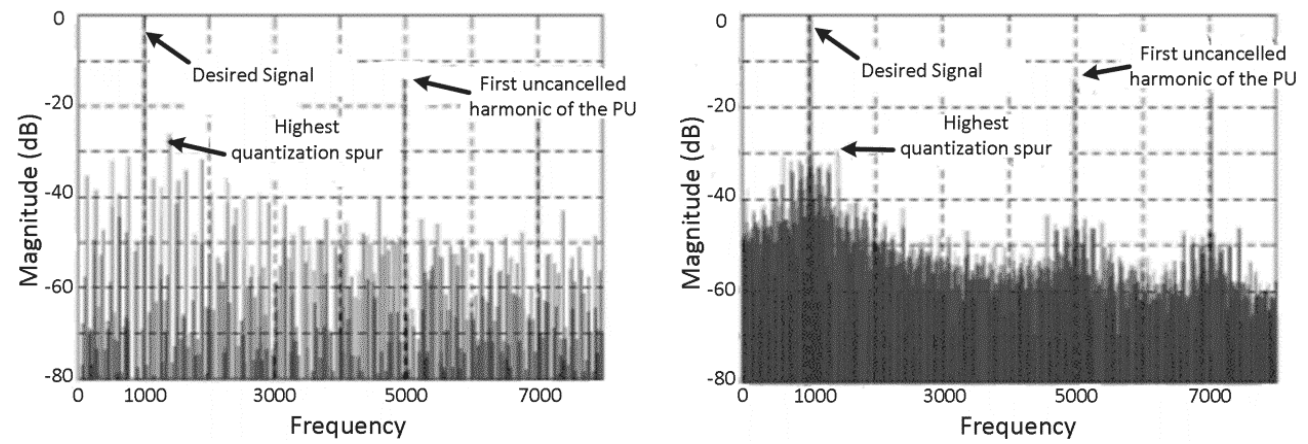

Figure 4.11 Output Spectrum of the model in Figure 4.10 for 6-paths and 3 bit quantization, using mixers as phase shifters and: a) a single tone (b) a 2-tone input.

The amplitude of the strongest up-converted frequency component produced due to the quantization process relative to the amplitude of the desired signal is determined through simulation. This criterion can also be termed as the spurious free dynamic range $\left(\mathrm{SFDR}_{\mathrm{Q}}\right)$ (Q due to quantization). These spurs were plotted for various quantization levels and number of paths. In order to determine the general trend of the $\mathrm{SFDR}_{\mathrm{Q}}$ variation with respect to the number of quantization bits a first order polynomial was fit through these points as shown in Figure 4.12. The slope of the $\mathrm{SFDR}_{\mathrm{Q}}$ curve follows roughly $8 \mathrm{db} / \mathrm{bit}$ for single tone and around $10 \mathrm{~dB} / \mathrm{bit}$ for two tone inputs.
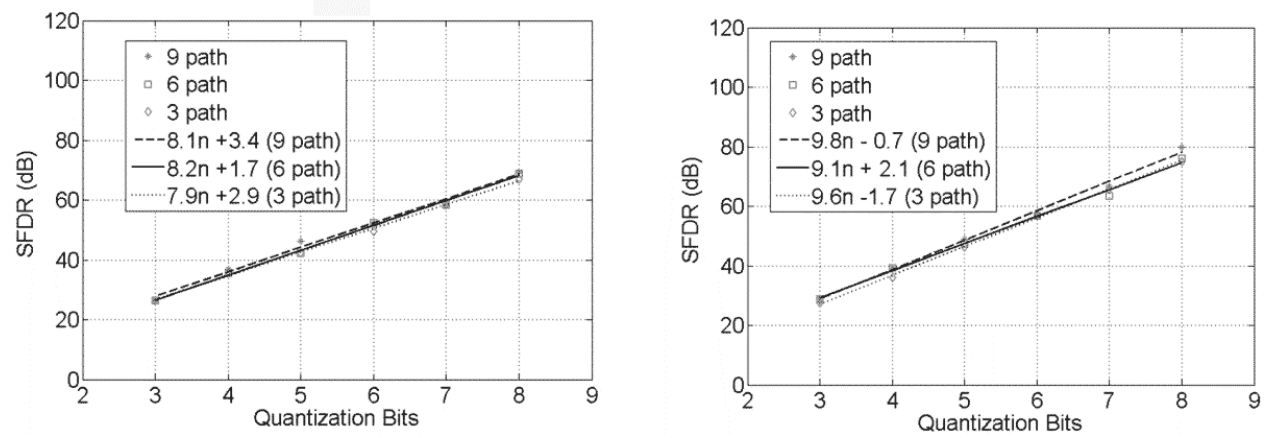

Figure 4.12 SFDR versus quantization bits (a) single tone (b) 2 tone

This pattern is close to the SFDR figures for ideal quantizers found in literature [7]. The increase in $\mathrm{SFDR}_{\mathrm{Q}}$ slope for two tones as compared to the single tone can be attributed to 
the decrease in correlation between the input signal and the quantization error. It can be concluded that in order to keep the SFDR due to limited DA resolution better than $50 \mathrm{~dB}$, a resolution of 7 bits or higher should be implemented for a polyphase multipath transmitter.

\subsection{DAC Replica Image Cancellation}

In literature we found that when multiple parallel DACs clocked with a phase shifted clock [10], [11] are utilized to reconstruct an analog signal, it can provide benefits in terms of DAC image suppression/ cancellation which can relax the reconstruction filter requirements. In this section we investigate the effect of using multiple parallel DACs on the DAC images. First we look at the scenario, where the outputs of the multiple DACs are combined directly at the output of the DAC without the multipath technique (no upconversion). Then the effect of combining the outputs in the presence of multipath (with upconversion) is also investigated.

In a conventional Digital to Analog Conversion process the image replicas of the digital input signal are always present at frequencies:

$$
f=i \cdot f_{s} \pm f_{\text {in }}
$$

where $f$ is the image replica frequency, $f_{s}$ is the sampling frequency and $f_{\text {in }}$ is the input signal frequency and $i$ is an integer.

The signal and the replicas are attenuated by the sinc $\left(\mathrm{f} / \mathrm{f}_{\mathrm{S}}\right)$ response of the inherent zero order hold function of the DAC. Where

$$
\operatorname{sinc}\left(\frac{f}{f_{s}}\right)=\frac{\sin \left(\frac{\pi \cdot f}{f_{s}}\right)}{\left(\frac{\pi \cdot f}{f_{s}}\right)}
$$

As an example a $10 \mathrm{MHz}$ signal sampled at $200 \mathrm{MHz}$ (sampling frequency $\mathrm{f}_{\mathrm{s}}$ ) will attenuate the first replica image occurring at $190 \mathrm{MHz}$ by $25 \mathrm{~dB}$. The higher frequency replicas are attenuated more. This is shown in the Figure 4.13.

\subsubsection{Suppression of DAC images due to summation of parallel DAC output}

It has been shown in literature that it is possible to cancel the DAC images [11] or attenuate DAC aliases [10], through a parallel DAC architecture with polyphase clocks. In order to be polyphase the phases need to be divided over the 360 degree period with equal 
distances in phase. For both of these techniques the outputs of the DACs are directly summed. In [10] the same input signal is fed to all the DACs, while polyphase DAC clocks running at $\mathrm{f}_{\mathrm{s}}$, clock the DAC's as shown in Figure 4.14.
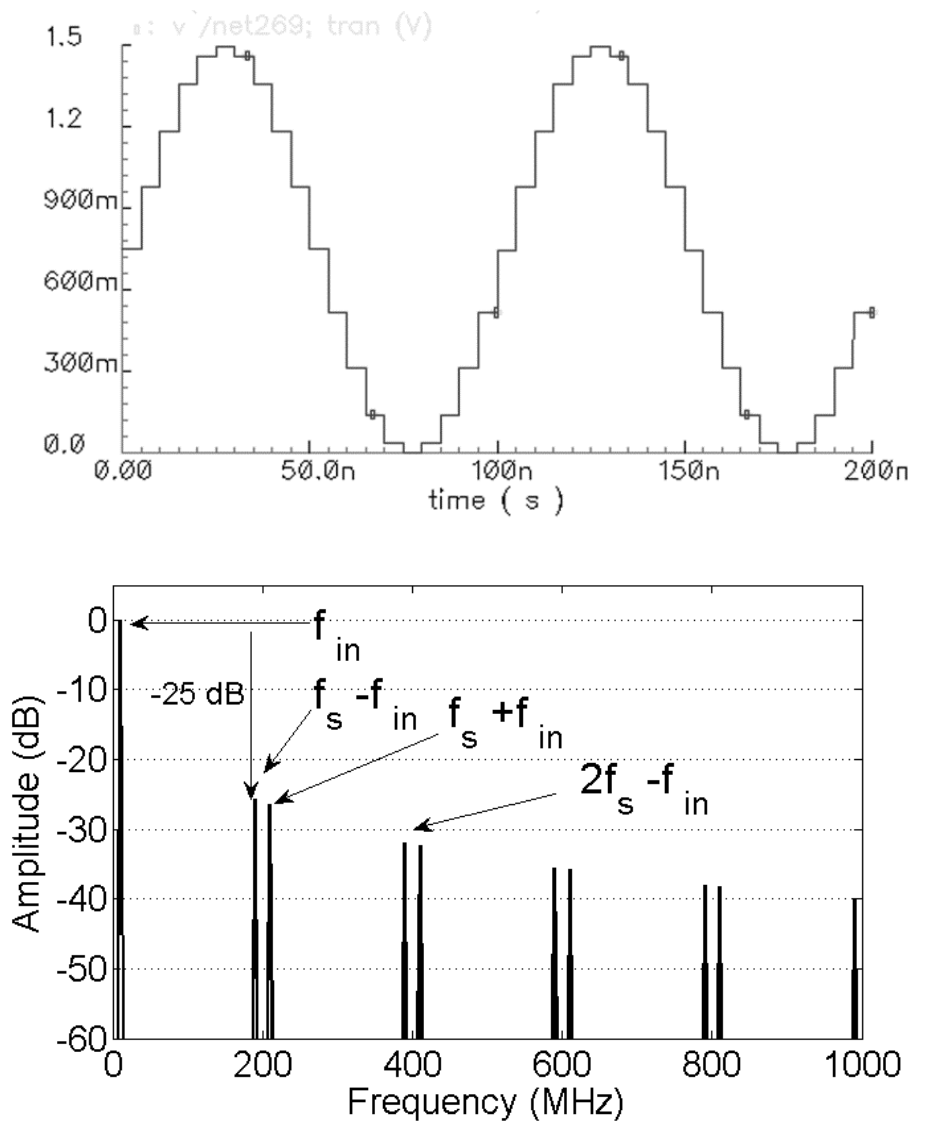

Figure 4.13.DAC output of 10MHz signal sampled at 200MHz, time domain (top) frequency domain (bottom)

The 2-path example of Figure 4.14 was simulated with a $10 \mathrm{MHz}$ signal sampled at 200 MHz. This results in the frequency spectrum shown in Figure 4.15. Images occurring around $\mathrm{f}_{\mathrm{s}}, 3 \mathrm{f}_{\mathrm{s}}, 5 \mathrm{f}_{\mathrm{s}} \ldots$ are suppressed by more than $22 \mathrm{~dB}$ as compared to the spectrum of Figure 4.13. The frequency spectrum of the output of the 2-path DAC can also be determined by (4.9) derived in [10], where $t_{d}$ is chosen as Ts/2 (the clock of DAC2 is Ts/2 shifted with respect to the clock of DAC1) and $\mathrm{f}$ is the frequency of the component whose magnitude is being calculated. 


$$
Y_{T}(f)=2 Y_{A}(f) \cdot e^{-j \cdot \pi \cdot f \cdot t_{d}} \cdot \cos \left(\pi \cdot f \cdot t_{d}\right)
$$

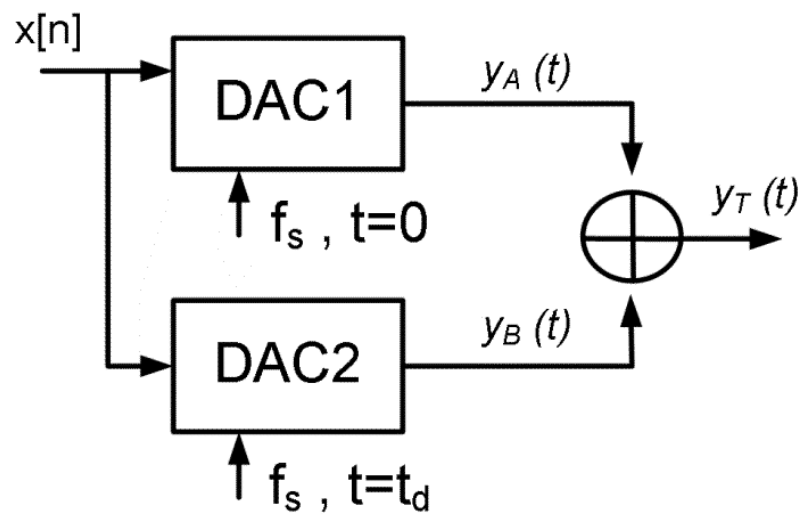

Figure 4.14 Block Diagram of a two phase DAC[10]

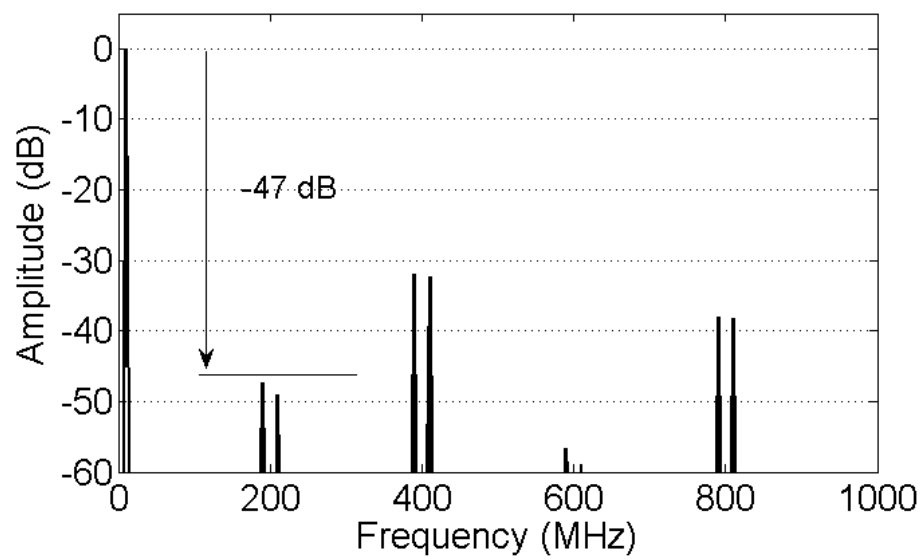

Figure 4.15 Attenuation of DAC images with two paths

If appropriate phases are selected it is possible to completely cancel a certain DAC image [10]. For example if a delay of $t_{0}(4.10)$ is given to the second path clock on top of the nominal delay of Ts/2 it can completely cancel the image at $f_{z}$, where $m$ is an odd integer as described in [10].

$$
t_{o}=\frac{m}{2 \cdot f_{z}}-\frac{1}{2 \cdot f_{s}}
$$

The technique discussed above is interesting if a specific DAC image has to be attenuated or cancelled. In order to cancel the DAC image over a wideband the technique in [11] is more interesting. Here the DACs are clocked with polyphase clocks running at $f_{s} / N(N$ is 
the number of parallel paths), but now the inputs are also delayed with respect to each other to cancel the images.

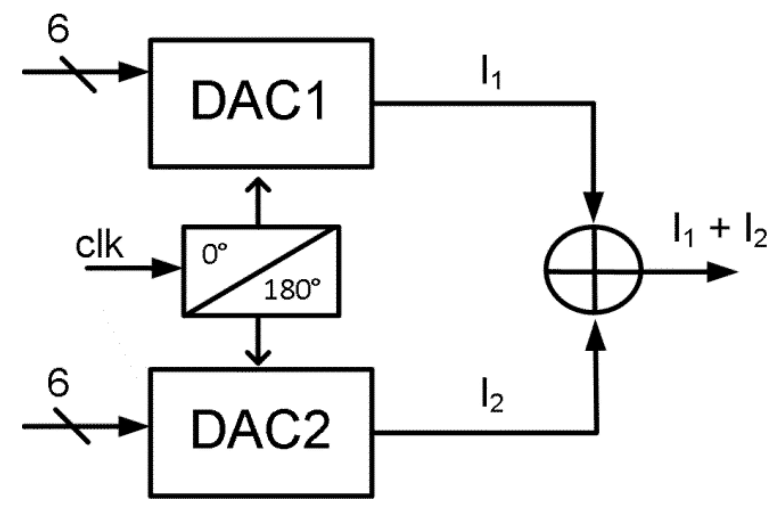

Figure 4.16 Two path DAC with input chosen such that image is cancelled[11]

Signal spectra for a PP-DAC
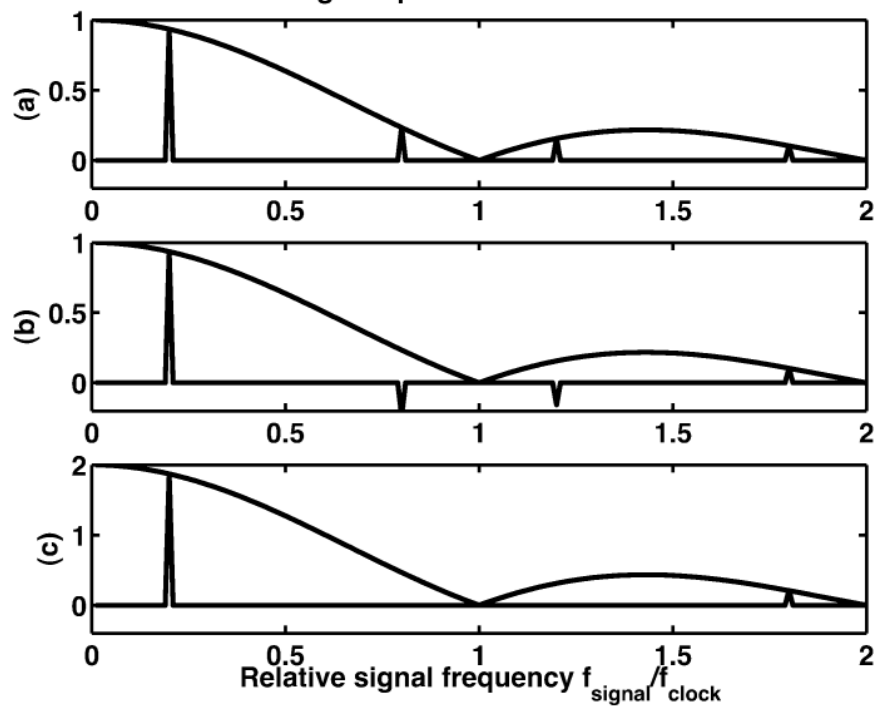

Figure 4.17. Frequency Spectra for the circuit in fig.4.16: DAC1 output (top); DAC2 output (middle); Sum of DAC1 and DAC2 (bottom)[11].

Figure 4.16 shows the block diagram of the parallel DAC system, while Figure 4.17 shows the output spectrum after summation. As can be seen in the figure, the first two images have anti-phase alignment, so after summation they are cancelled. Equation (4.11) calculates the frequency spectrum of the first DAC image after summation [11]. 


$$
Y_{P P D A, \text { image } 1}(f)=\operatorname{sinc}\left(f * T_{S}\right) *\left(1+e^{-j 2 \pi * \frac{\Delta T_{0}}{T_{S}}}\right) * W\left(f-1 / T_{S}\right)
$$

The architecture of Figure 4.16 was simulated with a $10 \mathrm{MHz}$ signal sampled at $200 \mathrm{MHz}$. Comparing the results in Figure 4.18 with those in Figure 4.15 we see that the DAC images occurring around $\mathrm{f}_{\mathrm{s}}(200 \mathrm{MHz}), 3 \mathrm{f}_{\mathrm{s}}(600 \mathrm{MHz})$ and $5 \mathrm{f}_{\mathrm{s}}(1 \mathrm{GHz})$ are completely cancelled. In both these techniques the clock signal of the parallel DACs is polyphase in nature. For $\mathrm{N}$ parallel DACs, the clock of the $\mathrm{n}^{\text {th }}$ DAC is phase shifted by $\mathrm{T}_{\mathrm{s}} / \mathrm{n}$, so a polyphase DAC clock would be required to drive $\mathrm{N}$ parallel DACs. In conclusion, the literature has shown, that when the output of parallel DACs, clocked by a polyphase clock, is added, it has the advantages for DAC image suppression/ cancellation.

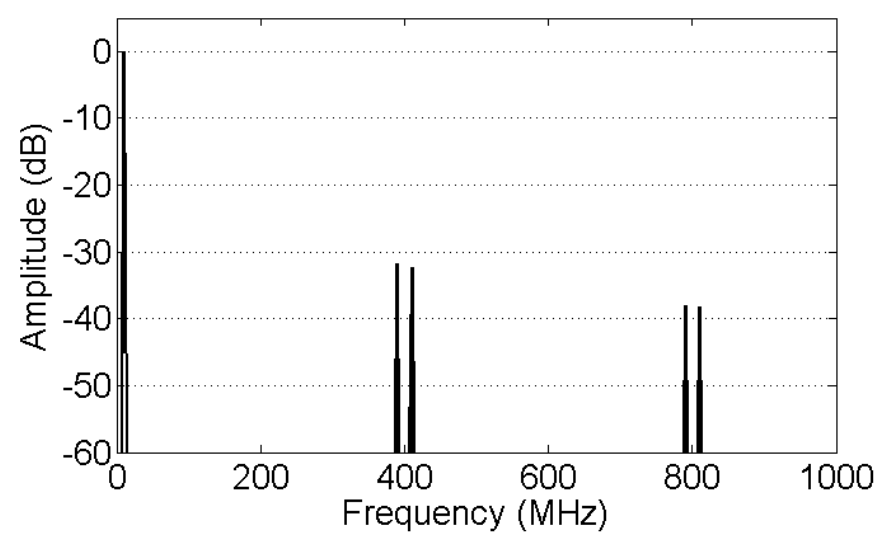

Figure 4.18 Spectrum after DAC image cancellation

\subsubsection{Suppression of DAC images in Polyphase Multipath Upconversion}

In the previous section we saw that some DAC images can be cancelled or suppressed at baseband (output of DAC) by using parallel DACs with a phase shifted clock. In this section we investigate what happens to the DAC images in the presence of the polyphase multipath upconversion technique. Concretely we want to know whether DAC images can be cancelled or suppressed using the multipath technique.

The Polyphase multipath upconverter requires multiphase baseband inputs to drive the multiple mixers, whose output currents are added at RF. This up-conversion requires two sets of phase shifts; one in digital and the other via polyphase mixer LO signals. The multiphase inputs can be generated from multiple DACs. In the absence of reconstruction 
filtering, and with the assumption that the DAC is perfectly linear, the input to the PU will contain not only the desired signal at frequency $f_{\text {in, }}$, but also images around the DAC frequency of $f_{s}$ and multiples of $f_{s}$.

The output spectrum of the upconversion is different depending on how the parallel DACs are clocked.

The following cases are discussed depending on how the DACs are clocked.

1. A single clock driving all the DACs

2. Polyphase clocks driving the DACs

After comparing the output spectrum of both these cases, it can be decided which scenario has better properties in terms of DAC image cancellation and whether using a polyphase DAC clock provides any benefits over using the same DAC clock in a polyphase multipath upconversion.

Case I: All DACs are clocked by a single sampling clock.

The system used to analyze the effect of phase shifts before and after the DAC is shown in Figure 4.19. The first set of blocks before the DAC represent the input to the DAC. Here the digital input to the DAC consists of not only the fundamental signal at $f_{\text {in }}$ but also the images around the sampling frequency $\left(\mathrm{f}_{\mathrm{s}}\right)$. We investigate what happens to these frequency components as they undergo phase shifts before the DAC and a second set of phase shifts after the DAC before being summed together.

As seen in Figure 4.19, the fundamental component undergoes a phase shift of $\varphi$ in the $1^{\text {st }}$ path and subsequently in the N-1 path the fundamental component undergoes a phase shift of $(\mathrm{N}-1)^{*} \varphi$. We know from digital processing theory [12] that when the fundamental component (at $f_{\text {in }}$ ) of a digital signal undergoes a phase shift of $\varphi$, its image occurring at $f_{s^{-}}$ $f_{\text {in }}$ will undergo a phase shift of $-\varphi$ with respect to the original, this frequency spectrum has been shown in the first set of phase shift blocks in Figure 4.19, with the phase indicated on top of each frequency component. 


\section{Phase Shift}

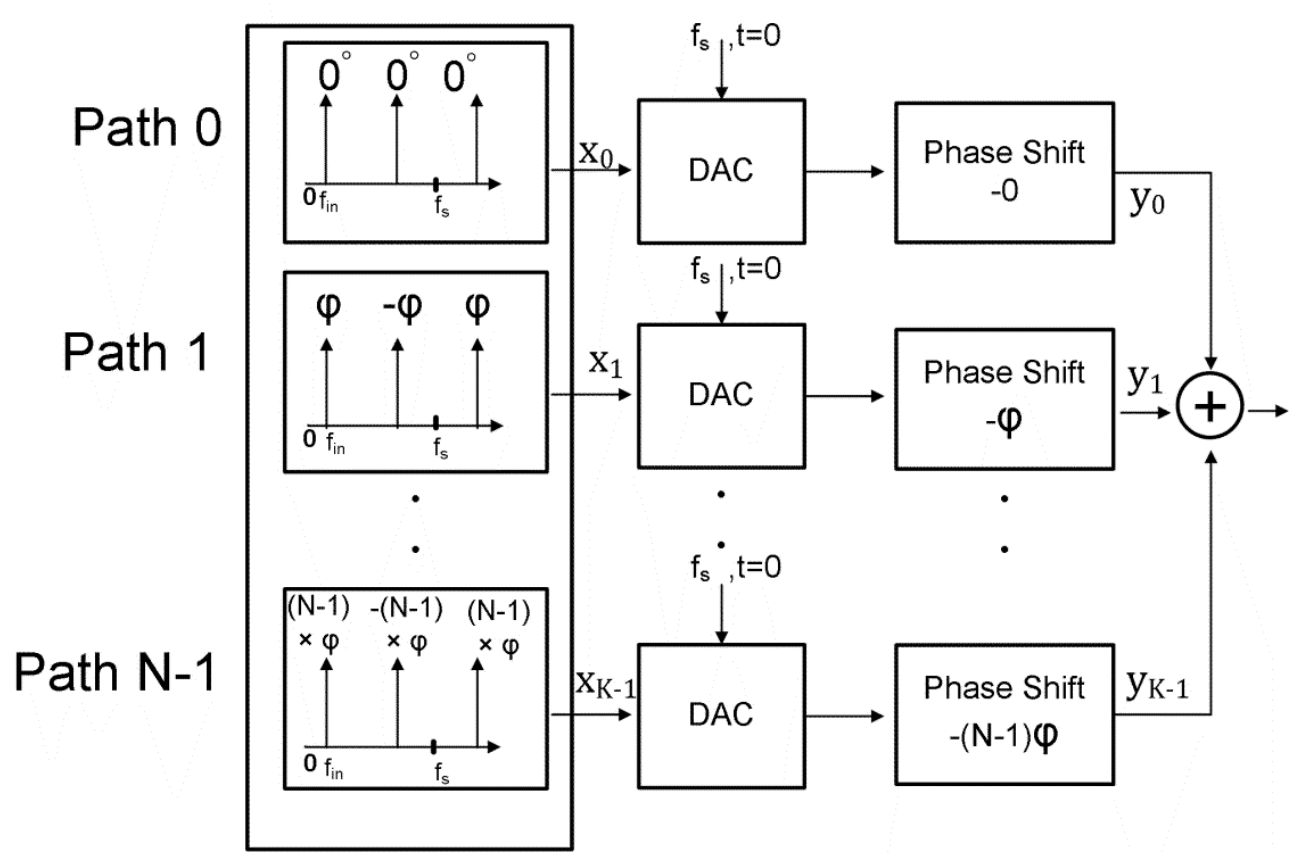

Figure 4.19. Multipath Technique with parallel DACs, clocked by a single sample clock.

Mathematically we can model this as follows: For simplicity we assume that the input is a set of sinusoids represented by the input signal $f_{\text {in }}$ and the first image at $f_{s}-f_{\text {in }}$. The second set of phase shifts are assumed to be provided by multiplication with a single sinusoid at frequency $\mathrm{f}_{\mathrm{LO}}$. The DACs are also assumed ideal meaning that they do not add any distortion to the signal passing through it. The zero order hold response of the ideal DAC which attenuates the images is assumed to affect all the DACs in the same way and thus maintains the phase relationship, so that is also not considered in the following equations. $\mathrm{x}_{0} \ldots \mathrm{x}_{\mathrm{K}-1}$ are the outputs of the first set of phase shifters, while $\mathrm{y}_{0} . . \mathrm{y}_{\mathrm{K}-1}$ are outputs of the second set of phase shifters. The location of the different frequency terms in the frequency spectrum can be seen in Figure 4.13.

$$
\begin{aligned}
& x_{0}=\cos \left(\omega_{i n} t\right)+\cos \left(\omega_{f_{s}-f_{i n}} t\right)+\ldots \\
& x_{1}=\cos \left(\omega_{i n} t+\frac{360^{\circ}}{N}\right)+\cos \left(\omega_{f_{s}-f_{i n}} t-\frac{360^{\circ}}{N}\right)+\ldots .
\end{aligned}
$$




$$
x_{K-1}=\cos \left(\omega_{i n} t+\frac{360^{\circ}}{N} \times(K-1)\right)+\cos \left(\omega_{f_{s}-f_{i n}} t-\frac{360^{\circ}}{N} \times(K-1)\right)+.
$$

After upconversion by the $\mathrm{LO}$ frequency $\mathrm{f}_{\mathrm{LO}}$, the following terms result:

$$
\begin{aligned}
& y_{0}=1 / N \cdot\left[\cos \left(\omega_{L O} t+\omega_{i n} t\right)+\cos \left(\omega_{L O} t-\omega_{i n} t\right)+\right. \\
& \left.\cos \left(\omega_{L O} t+\omega_{f_{s}-f_{i n}} t\right)+\cos \left(\omega_{L O} t-\omega_{f_{s}-f_{i n}} t\right)\right]+\ldots \\
& y_{2}=1 / N \cdot\left[\cos \left(\omega_{L O} t+\omega_{i n} t\right)+\cos \left(\omega_{L O} t-\omega_{i n} t-\frac{720^{\circ}}{N}\right)+\right. \\
& \left.\cos \left(\omega_{L O} t+\omega_{f_{s}-f_{i n}} t-\frac{720^{\circ}}{N}\right)+\cos \left(\omega_{L O} t-\omega_{f_{s}-f_{i n}} t\right)\right]+\ldots \\
& y_{K-1}=1 / N \cdot\left[\cos \left(\omega_{L O} t+\omega_{i n} t\right)+\cos \left(\omega_{L O} t-\omega_{i n} t-\frac{720^{\circ}}{N} \times(K-1)\right)+\right. \\
& \left.\cos \left(\omega_{L O} t+\omega_{f_{s}-f_{i n}} t-\frac{720^{\circ}}{N} \times(K-1)\right)+\cos \left(\omega_{L O} t-\omega_{f_{s}-f_{i n}} t\right)\right]+\ldots
\end{aligned}
$$

Where $\mathrm{K}=1,2 \ldots \mathrm{N}$. Choosing $\mathrm{N}=3,4,5 \ldots$, it can be seen that after summation the frequency components at $\mathrm{f}_{\mathrm{LO}}-\mathrm{f}_{\mathrm{in}}$, and $\mathrm{f}_{\mathrm{LO}}+\mathrm{f}_{\mathrm{s}}-\mathrm{f}_{\text {in }}$ (image of the fundamental, around $\mathrm{f}_{\mathrm{s}}$ ) will be cancelled, while the frequency component at $\mathrm{f}_{\mathrm{LO}}-\left(\mathrm{f}_{\mathrm{s}}-\mathrm{f}_{\text {in }}\right)$ (image of the fundamental, around $\mathrm{f}_{\mathrm{s}}$ ) is not cancelled. The location of these frequency components in the frequency spectrum can be seen for a one path upconversion in Figure 4.21 (top).

Simulating a 4-path system in Figure 4.20, we obtain the spectrum shown in Figure 4.21 (bottom), where one of the upconverted images around $\omega_{\mathrm{LO}}+\omega_{\mathrm{fs}}$ is cancelled. For comparison purposes a one path spectrum is also shown in Figure 4.21. Here the baseband frequency was at $10 \mathrm{MHz}$ and $\mathrm{f}_{\mathrm{s}}$ at $200 \mathrm{MHz}$ while the $\mathrm{LO}$ was at $10 \mathrm{GHz}$ (chosen high so that the image components can be clearly seen and not just those that fold back from $0 \mathrm{~Hz}$ ). Simulating with different number of paths we observe the same effect that whenever all the DACs in a multipath architecture are clocked with the same sample frequency, one of the images around $\mathrm{f}_{\mathrm{s}}$ in the upconverted spectrum will be cancelled. This cancellation of the image will give some benefit in terms of cleaner output spectrum in the presence of relaxed 
baseband filtering but since one of the DAC image still appears on the other side of the LO as seen in the equations (after summation), the benefit in terms of relaxed baseband filtering will be limited. More benefit can be achieved (as seen in Case II) if multiphase DAC clocks are utilized as was the case in section 4.3.1, but now in the context of multipath technique as discussed next.

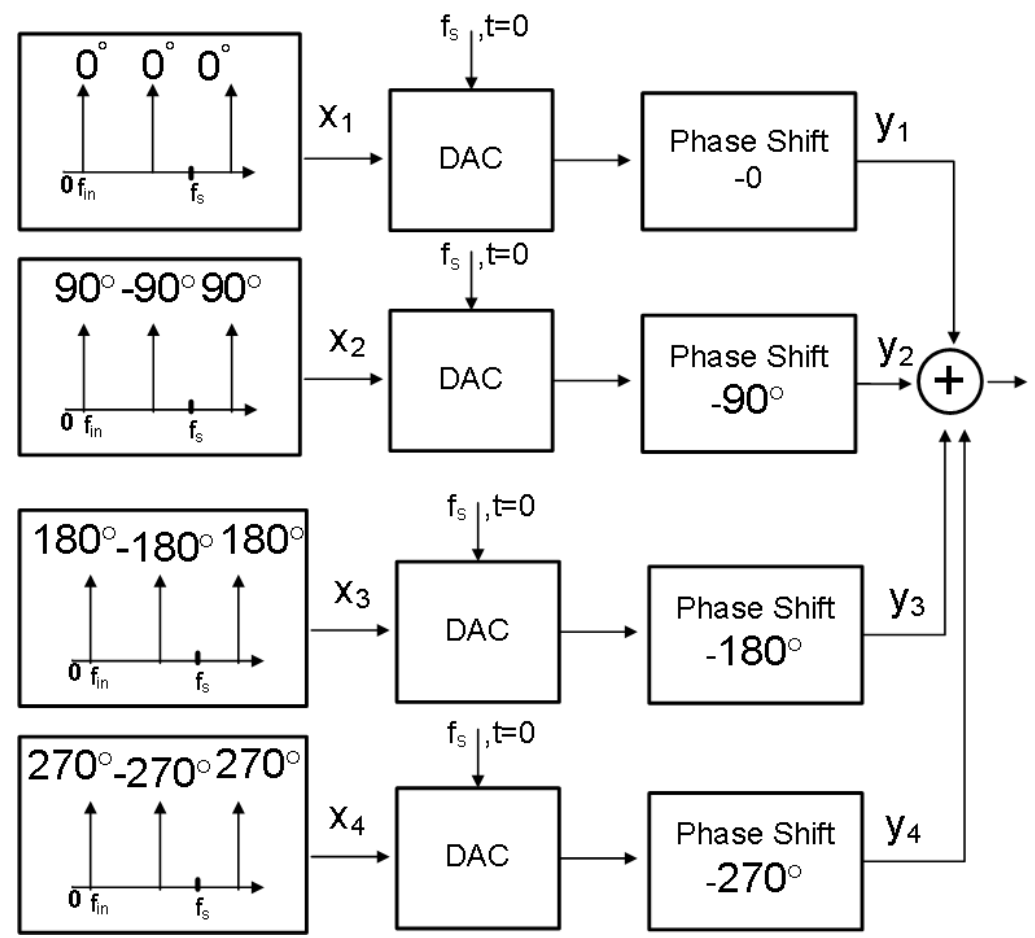

Figure 4.20. 4-Path upconversion with parallel DACs, clocked by a single sample clock 

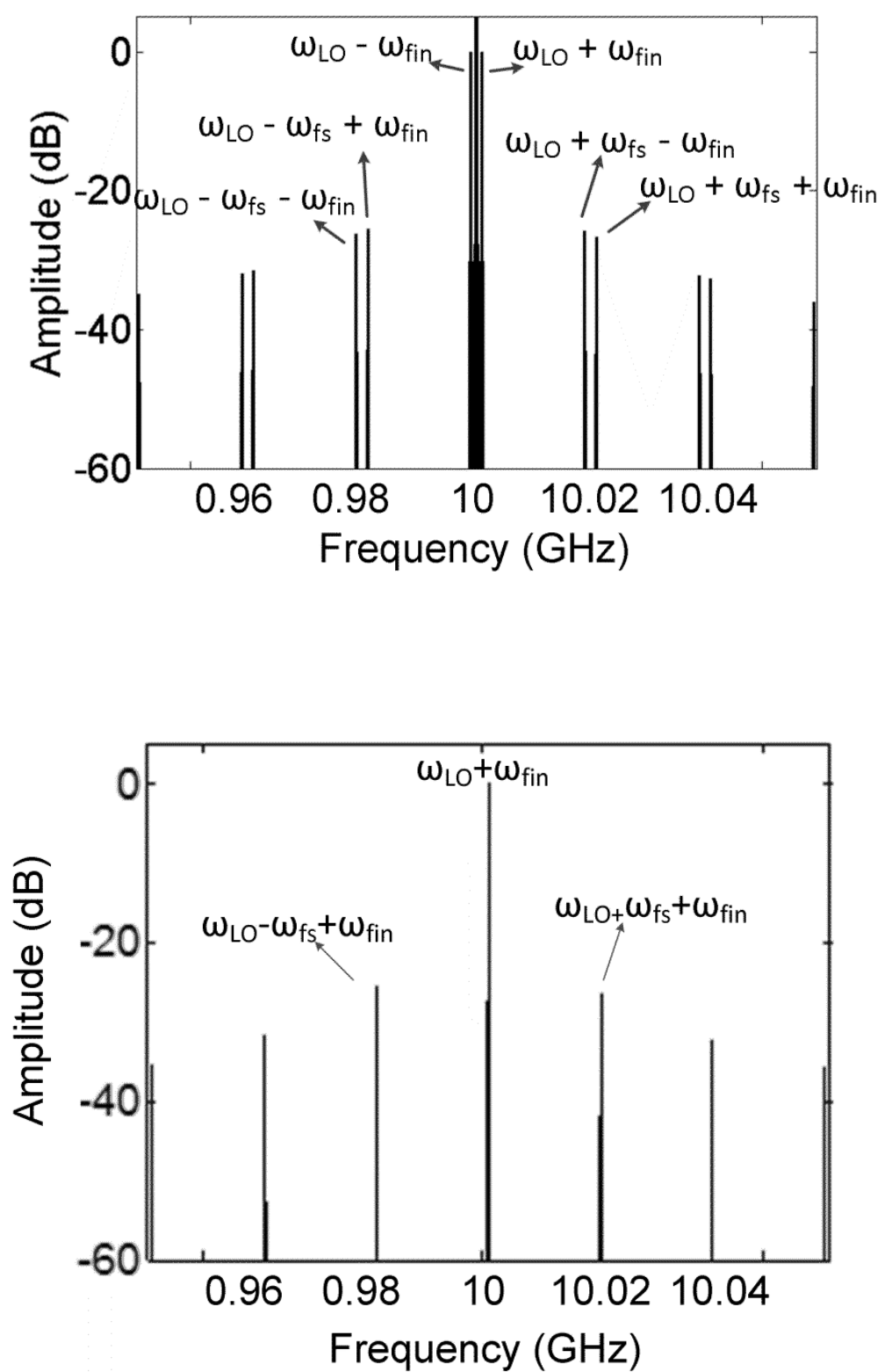

Figure 4.21 Spectrum of one path upconversion (top) 4 path upconversion with DAC images at $\omega_{L O}+\omega_{f s}-\omega_{f i n}$ and $\omega_{L O}-\omega_{f s}-\omega_{f i n}$ among others being cancelled (bottom) (LO at 10GHz). 
Case II: DACs are clocked by a polyphase sample clock.

In section 4.3.1, it was shown that when multiple parallel DACs are clocked with a multiphase clock and their outputs are combined, there are benefits in terms of DAC image cancellation/ suppression. In the present scenario we look at the same case, but now not only with polyphase baseband inputs for all the DACs but also polyphase LO which provides the phase shift after the DAC shown in Figure 4.22. The DAC clock in the $1^{\text {st }}$ path will have a delay of $\mathrm{T}_{\mathrm{s}} / \mathrm{N}$ with respect to the DAC clock in path 0 , while there will be a delay of $\frac{T_{s}}{N} \cdot(N-1)$ in the $(\mathrm{N}-1)^{\text {th }}$ path as shown in Figure 4.22. Unlike the first case (Case I), here the DAC itself will introduce a phase shift to the input signal due to the polyphase nature of the sampling clock.

\section{Phase Shift}

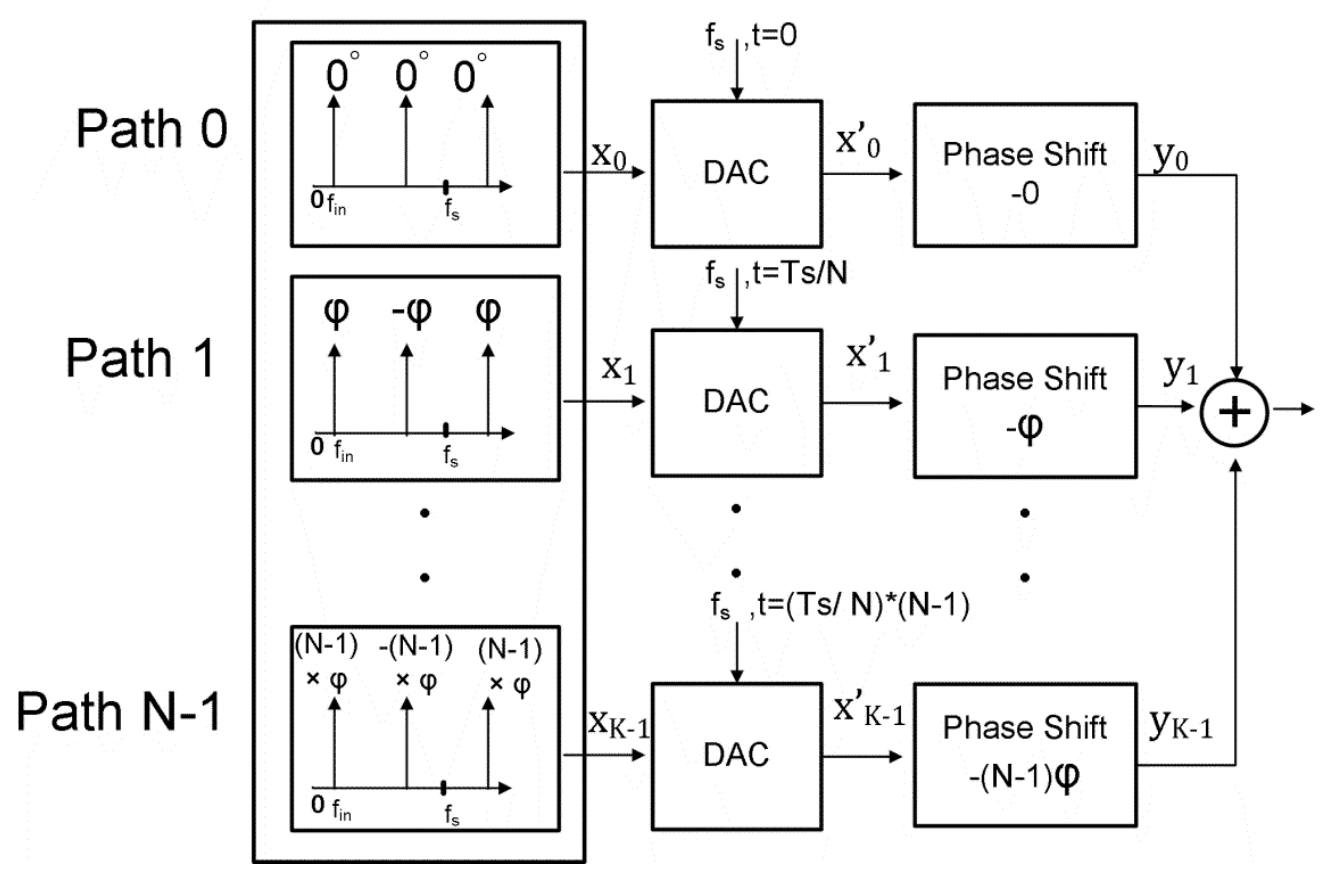

Figure 4.22. Multipath up-conversion, DACs driven with polyphase clock.

Mathematically we can write the following equations to describe the first few frequency components present at the input to the DAC. We only consider a single tone input for simplicity, but the analysis can be extended to include multiple tones as well.

$$
x_{0}=\cos \left(\omega_{i n} t\right)+\cos \left(\omega_{f_{s}-f_{i n}} t\right)+\cos \left(\omega_{f_{s}+f_{i n}} t\right)+\cos \left(\omega_{2 f_{s}-f_{i n}} t\right)+. .
$$




$$
\begin{aligned}
& x_{1}=\cos \left(\omega_{i n} t+\frac{360^{\circ}}{N}\right)+\cos \left(\omega_{f_{s}-f_{\text {in }}} t-\frac{360^{\circ}}{N}\right) \\
& +\cos \left(\omega_{f_{s}+f_{\text {in }}} t+\frac{360^{\circ}}{N}\right)+\cos \left(\omega_{2 f_{s}-f_{\text {in }}} t-\frac{360^{\circ}}{N}\right)+\ldots \\
& x_{K-1}=\cos \left(\omega_{i n} t+\frac{360^{\circ}}{N} \times(K-1)\right)+\cos \left(\omega_{f_{s}-f_{\text {in }}} t-\frac{360^{\circ}}{N} \times(K-1)\right)+ \\
& \cos \left(\omega_{f_{s}+f_{\text {in }}} t+\frac{360^{\circ}}{N} \times(K-1)\right)+\cos \left(\omega_{2 f_{s}-f_{\text {in }}} t-\frac{360^{\circ}}{N} \times(K-1)\right)+\ldots
\end{aligned}
$$

The following equations describe the output of the DAC, neglecting the sinc response at the moment, and only considering the phase variation due to the polyphase DAC clock.

$$
x_{0}^{\prime}=\cos \left(\omega_{i n} t\right)+\cos \left(\omega_{f_{s}-f_{i n}} t\right)+\cos \left(\omega_{f_{s}+f_{i n}} t\right)+\cos \left(\omega_{2 f_{s}-f_{i n}} t\right)+. .
$$

We take $\mathrm{OS}=\mathrm{f}_{\mathrm{s}} / \mathrm{f}_{\text {in }}$

$$
\begin{aligned}
& x_{1}^{\prime}=\cos \left(\omega_{i n} t+\frac{360^{\circ}}{N}+\left(\frac{360^{\circ}}{N \cdot O S}\right)\right)+ \\
& \cos \left(\omega_{f_{s}-f_{i n}} t-\frac{360^{\circ}}{N}+\left(\frac{360^{\circ}}{N \cdot O S}\right) \cdot(O S-1)\right) \\
& +\cos \left(\omega_{f_{s}+f_{i n}} t+\frac{360^{\circ}}{N}+\left(\frac{360^{\circ}}{N \cdot O S}\right) \cdot(O S+1)\right)+ \\
& \cos \left(\omega_{2 f_{s}-f_{i n}} t-\frac{360^{\circ}}{N}+\left(\frac{360^{\circ}}{N \cdot O S}\right) \cdot(2 \cdot O S-1)\right)+\ldots
\end{aligned}
$$

Comparing the above equation with 4.22 , we see that for the fundamental input signal, aside from the nominal phase shift of $360^{\circ} / \mathrm{N}$ required by the polyphase multipath technique, there is an additional phase shift of $\frac{360^{\circ}}{N \cdot O S}$ caused due to the delay of the multiphase DAC sampling. The first DAC image occurring at $\mathrm{f}_{\mathrm{s}}-\mathrm{f}_{\text {in }}$, will undergo a phase shift of $\frac{360^{\circ}}{N \cdot O S} \times(\mathrm{OS}-1)$, as it occurs at (OS-1) times the input signal. 
The above equation can be extended to the $(\mathrm{K}-1)^{\text {th }}$ path as follows.

$$
\begin{aligned}
& x_{K-1}^{\prime}=\cos \left(\omega_{i n} t+\frac{360^{\circ}}{N} \times(K-1)+\left(\frac{360^{\circ}}{N \cdot O S} \times(K-1)\right)\right)+ \\
& \cos \left(\omega_{f_{s}-f_{\text {in }}} t-\frac{360^{\circ}}{N \cdot O S} \times(K-1)+\left(\frac{360^{\circ}}{N \cdot O S} \times(K-1)\right) \cdot(O S-1)\right)+ \\
& \cos \left(\omega_{f_{s}+f_{\text {in }}} t+\frac{360^{\circ}}{N \cdot O S} \times(K-1)+\left(\frac{360^{\circ}}{N \cdot O S} \times(K-1)\right) \cdot(O S+1)\right)+ \\
& \cos \left(\omega_{2 f_{s}-f_{\text {in }}} t-\frac{360^{\circ}}{N \cdot O S} \times(K-1)+\left(\frac{360^{\circ}}{N \cdot O S} \times(K-1)\right) \cdot(2 \cdot O S-1)\right)+.
\end{aligned}
$$

After upconversion by a single tone at frequency $\omega_{\mathrm{LO}}$, the following equation results

$$
\begin{aligned}
& y_{0}=\cos \left(\omega_{L O} t+\omega_{i n} t\right)+\cos \left(\omega_{L O} t-\omega_{i n} t\right)+\cos \left(\omega_{L O} t+\omega_{f_{s}-f_{i n}} t\right)+ \\
& \cos \left(\omega_{L O} t-\omega_{f_{s}-f_{\text {in }}} t\right)+\cos \left(\omega_{L O} t+\omega_{f_{s}+f_{\text {in }}} t\right)+ \\
& +\cos \left(\omega_{L O} t-\omega_{f_{s}+f_{i n}} t\right)+\cos \left(\omega_{L O} t+\omega_{2 f_{s}-f_{i n}} t\right)++\cos \left(\omega_{L O} t-\omega_{2 f_{s}-f_{\text {in }}} t\right) . . \\
& y_{1}=\cos \left(\omega_{L O} t+\omega_{i n} t+\left(\frac{360^{\circ}}{N \cdot O S}\right)+\right. \\
& \cos \left(\omega_{L O} t-\omega_{\text {in }} t-\frac{720^{\circ}}{N}-\left(\frac{360^{\circ}}{N \cdot O S}\right)+\right. \\
& \cos \left(\omega_{L O} t+\omega_{f_{s}-f_{\text {in }}} t-\frac{720^{\circ}}{N}+\left(\frac{360^{\circ}}{N \cdot O S}\right) \cdot(O S-1)\right)+ \\
& \cos \left(\omega_{L O} t-\omega_{f_{s}-f_{i n}} t-\left(\frac{360^{\circ}}{N \cdot O S}\right) \cdot(O S-1)\right)+ \\
& \cos \left(\omega_{L O} t+\omega_{f_{s}+f_{i t}} t+\left(\frac{360^{\circ}}{N \cdot O S}\right) \cdot(O S+1)\right)+ \\
& \cos \left(\omega_{L O} t-\omega_{f_{s}+f_{i n}} t-\frac{720^{\circ}}{N}-\left(\frac{360^{\circ}}{N \cdot O S}\right) \cdot(O S+1)\right)+ \\
& \cos \left(\omega_{L O} t+\omega_{2 f_{s}-f_{\text {in }}} t-\frac{720^{\circ}}{N}+\left(\frac{360^{\circ}}{N \cdot O S}\right) \cdot(2 \cdot O S-1)\right)+ \\
& \cos \left(\omega_{L O} t-\omega_{2 f_{s}-f_{\text {in }}} t-\left(\frac{360^{\circ}}{N \cdot O S}\right) \cdot(2 \cdot O S-1)\right)+\ldots .
\end{aligned}
$$




$$
\begin{aligned}
& y_{K-1}=\cos \left(\omega_{L O} t+\omega_{i n} t+\left(\frac{360^{\circ}}{N \cdot O S}\right) \times(K-1)+\right. \\
& \left.\cos \left(\omega_{L O} t-\omega_{i n} t-\frac{720^{\circ}}{N} \times(K-1)\right)-\left(\frac{360^{\circ}}{N \cdot O S} \times(K-1)\right)\right)+ \\
& \cos \left(\omega_{L O} t+\omega_{f_{s}-f_{i n}} t-\frac{720^{\circ}}{N} \times(K-1)\right)+ \\
& \left.\left(\frac{360^{\circ}}{N \cdot O S} \times(K-1)\right) \cdot(O S-1)\right)+ \\
& \cos \left(\omega_{L O} t-\omega_{f_{s}-f_{i n}} t-\left(\frac{360^{\circ}}{N \cdot O S} \times(K-1)\right) \cdot(O S-1)\right)+ \\
& \cos \left(\omega_{L O} t+\omega_{f_{s}+f_{i n}} t+\left(\frac{360^{\circ}}{N \cdot O S} \times(K-1)\right) \cdot(O S+1)\right)+ \\
& \cos \left(\omega_{L O} t-\omega_{f_{s}+f_{i n}} t-\frac{720^{\circ}}{N} \times(K-1)\right) \\
& \left.+\left(\frac{360^{\circ}}{N \cdot O S} \times(K-1)\right) \cdot(O S+1)\right) \\
& \cos \left(\omega_{L O} t+\omega_{2 f_{s}-f_{i n}} t-\frac{720^{\circ}}{N} \times(K-1)\right. \\
& \left.+\left(\frac{360^{\circ}}{N \cdot O S} \times(K-1)\right) \cdot(2 \cdot O S-1)\right)+ \\
& \cos \left(\omega_{L O} t-\omega_{2 f_{s}-f_{i n}} t-\left(\frac{360^{\circ}}{N \cdot O S} \times(K-1)\right) \cdot(2 \cdot O S-1)\right) \ldots \\
&
\end{aligned}
$$

If we assume $2 \cdot \mathrm{OS}>>1$, so that $(2 \cdot \mathrm{OS}-1) \sim 2 \cdot \mathrm{OS}$ 


$$
\begin{aligned}
& y_{K-1} \approx \cos \left(\omega_{L O} t+\omega_{i n} t+\left(\frac{360^{\circ}}{N \cdot O S}\right) \times(K-1)+\right. \\
& \left.\cos \left(\omega_{L O} t-\omega_{i n} t-\frac{720^{\circ}}{N} \times(K-1)\right)-\left(\frac{360^{\circ}}{N \cdot O S} \times(K-1)\right)\right)+ \\
& \cos \left(\omega_{L O} t+\omega_{f_{s}-f_{i n}} t-\frac{720^{\circ}}{N} \times(K-1)\right)+ \\
& \left.\left(\frac{360^{\circ}}{N \cdot O S} \times(K-1)\right) \cdot(O S-1)\right)+ \\
& \cos \left(\omega_{L O} t-\omega_{f_{s}-f_{i n}} t-\left(\frac{360^{\circ}}{N \cdot O S} \times(K-1)\right) \cdot(O S-1)\right)+ \\
& \cos \left(\omega_{L O} t+\omega_{f_{s}+f_{i n}} t+\left(\frac{360^{\circ}}{N \cdot O S} \times(K-1)\right) \cdot(O S+1)\right)+ \\
& \cos \left(\omega_{L O} t-\omega_{f_{s}+f_{i n}} t-\frac{720^{\circ}}{N} \times(K-1)\right) \\
& \left.+\left(\frac{360^{\circ}}{N \cdot O S} \times(K-1)\right) \cdot(O S+1)\right)+ \\
& \cos \left(\omega_{L O} t+\omega_{2 f_{s}-f_{i n}} t\right)+ \\
& \cos \left(\omega_{L O} t-\omega_{2 f_{s}-f_{i n}} t-\left(\frac{720^{\circ}}{N} \times(K-1)\right)\right) \ldots
\end{aligned}
$$

So we see that the frequency components at $\omega_{L O}+\omega_{2 f_{s}-f_{\text {in }}}$ will have the same phase in each path and will not cancel in a polyphase multipath system but rather add up. This frequency component is also free of $\mathrm{N}$, therefore the number of paths will not effect this term and it will remain uncancelled.

The desired signal at $\omega_{L O}+\omega_{i n}$ will not align exactly in phase and all the other frequency components in (4.27) will not align exactly in anti phase and there would be some residual frequency components remaining. This is shown in the 4-path example in the next paragraph.

To understand better why cancellation is not achieved, the 4-path example shown in Figure 4.23 can be visualized. Here again it is assumed that the baseband frequency is at $10 \mathrm{MHz}$ and $\mathrm{f}_{\mathrm{s}}$ is at $200 \mathrm{MHz}$ while the $\mathrm{LO}$ is at $10 \mathrm{GHz}$. 


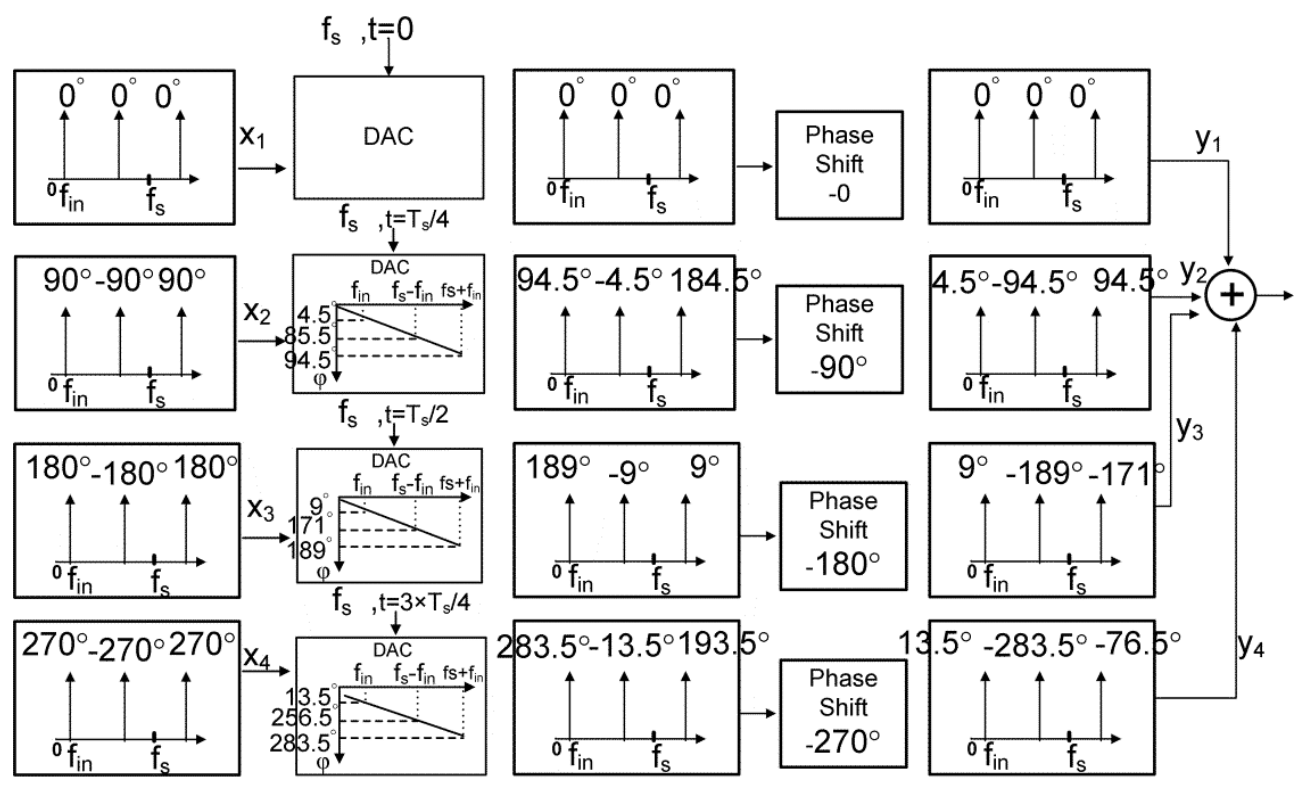

Figure 4.23. Multiphase upconversion where the DACs are also driven with polyphase clock.
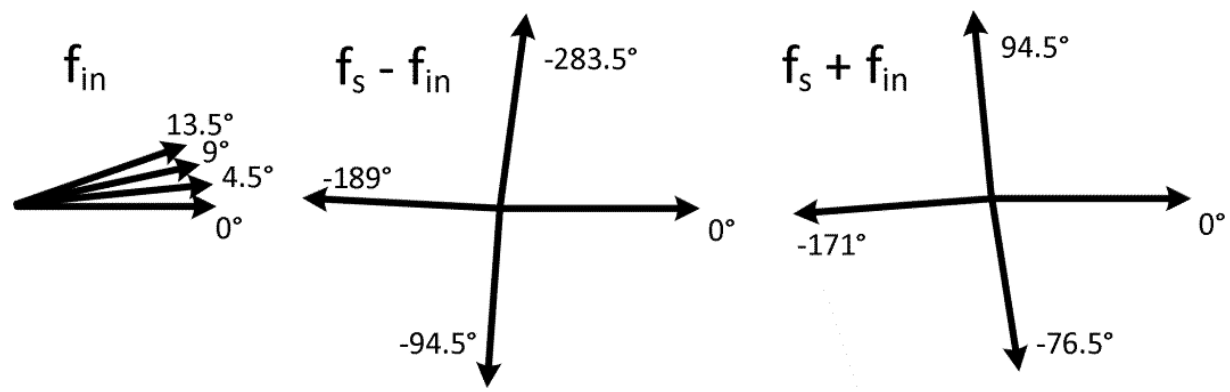

Figure 4.24. Phasor diagram for the frequency components at the summation point of Figure 4.23.

The DAC blocks also indicate the phase delay they cause at different frequencies (i.e. $\mathrm{f}_{\text {in }}, \mathrm{f}_{\mathrm{s}}$ $\mathrm{f}_{\text {in }}, \mathrm{f}_{\mathrm{s}}+\mathrm{f}_{\mathrm{in}}$ ) for a 20 times higher clock frequency than the input signal. One sample phase delay is equivalent to $\mathrm{Ts}_{\mathrm{s}}=360^{\circ} / 20=18^{\circ}$. This implies that $\mathrm{Ts} / 4=4.5^{\circ}$ (see eq.(4.23)). The fundamental signal in the first path will undergo a phase shift of $4.5^{\circ}$ after passing through the DAC, while the signal at $\mathrm{f}_{\mathrm{s}}-\mathrm{f}_{\text {in }}$ which is at 19 times higher in frequency will undergo a phase shift of $19 \times 4.5^{\circ}=85.5^{\circ}$. The phase change after each block is also shown. Just before summation it is seen that the fundamental components and also the images do not align exactly in anti phase as shown in Figure 4.24. Therefore there will be some residual frequency components (due to incomplete cancellation) in the frequency spectrum as predicted by (4.27). 
Figure 4.25 shows the simulated output spectrum of the system in Figure 4.23, where the upconverted images around $\omega_{\mathrm{LO}}+\omega_{\mathrm{fs}}$ are suppressed but not completely cancelled. Another disadvantage here is that the image of the fundamental at $\omega_{\mathrm{LO}}-\omega_{\text {fin }}$ is not completely cancelled due to the non-anti phase alignment of this image, due to the additional phase shift caused by the polyphase DAC sample clock. This drawback can be overcome by delay compensating the input signal such that it aligns in such a way that the image signal at $\omega_{\mathrm{LO}^{-}}$ $\omega_{\text {fin }}$ cancels out. This delay compensation is illustrated in the Figure 4.26 for a single tone, but can be extended for multiple tones as well.

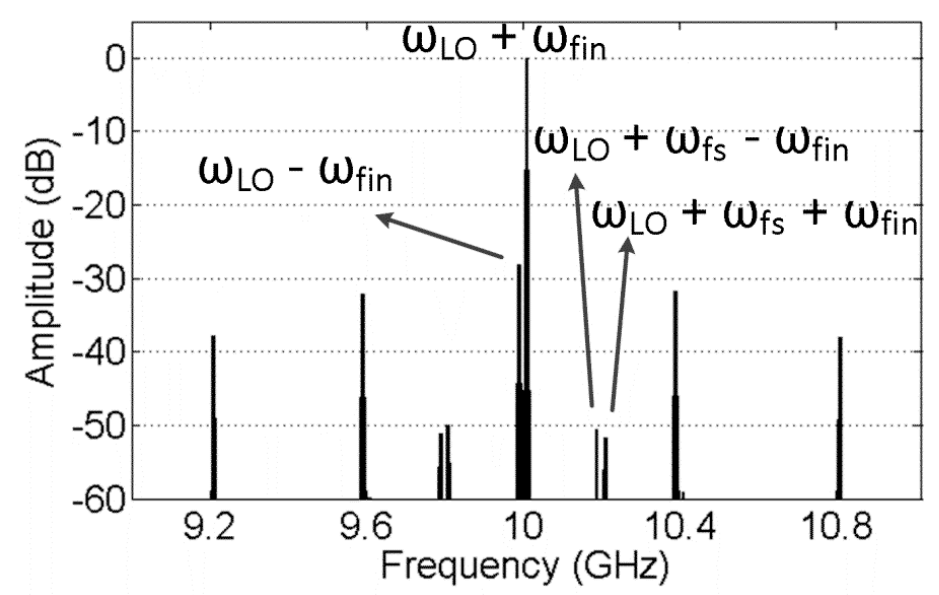

Figure 4.25. Output Spectrum of 4-path upconversion with DACs driven with multiphase sample clock.

As compared to Figure 4.23, the input signal is now also adjusted to compensate for the phase shift caused by the polyphase sample clock of the DACs. It can be seen that the fundamental component in the $2^{\text {nd }}, 3^{\text {rd }}$ and $4^{\text {th }}$ paths are compensated by $4.5^{\circ}, 9^{\circ}$ and $13.5^{\circ}$ respectively. These values can be calculated by putting $\mathrm{K}=2,3$ and $4, \mathrm{OS}=20$ and $\mathrm{N}=4$ in (4.26). Just before the summation, the fundamental components are now aligned in phase, which also enables the image at $\omega_{\mathrm{LO}}-\omega_{\text {fin }}$ to be cancelled.

The output spectrum for a 4-path upconverter is shown in Figure 4.27, while an 8-path upconversion spectrum is shown in Figure 4.28. The upconverted DAC image at $\omega_{\mathrm{LO}+} 2 \omega_{\mathrm{fs}^{-}}$ $\omega_{\text {fin }}$ remains uncancelled in both cases due to its in-phase addition after summation as predicted by (4.27). Simulation for different number of paths shows that this image remains uncancelled. A two-tone simulation (5MHz and $20 \mathrm{MHz}$ ) for an 8-path upconversion is also shown in Figure 4.29, to confirm that the delay compensation technique also works for multi-tones. 


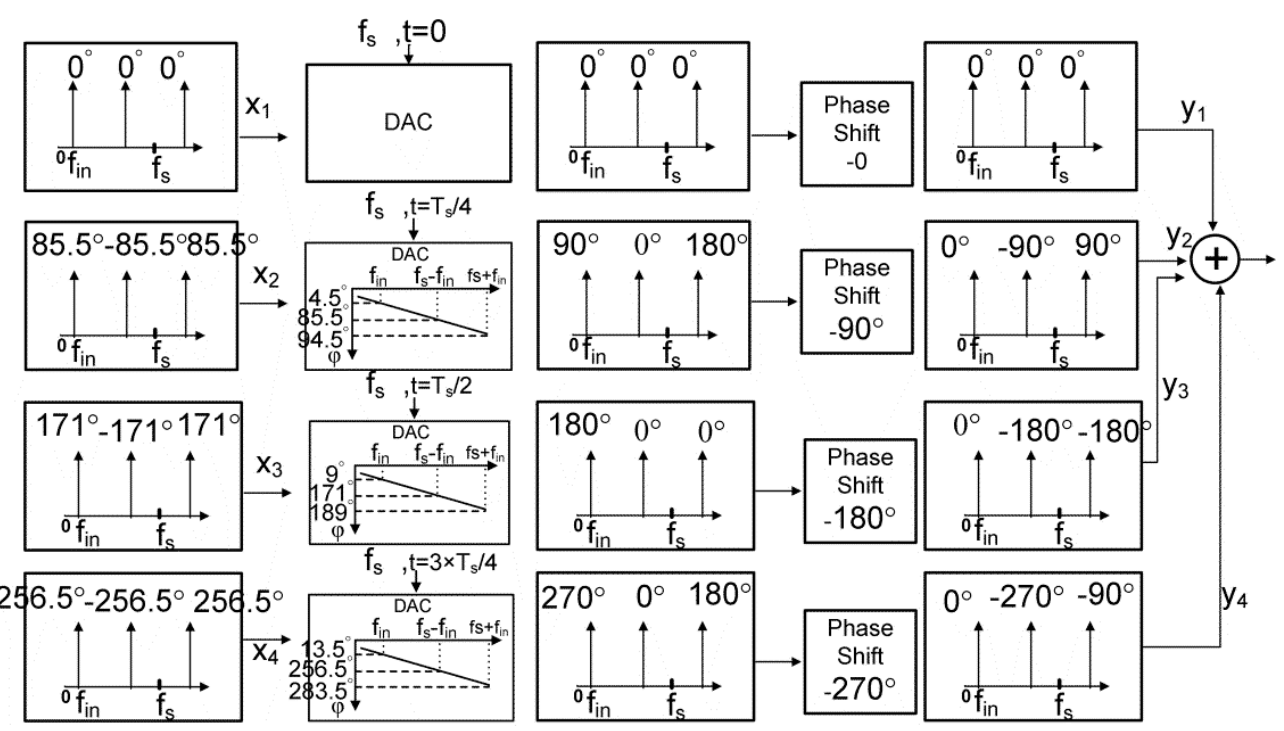

Figure 4.26 Multiphase upconversion where the DACs are driven with multiphase clock and the inputs are delay compensated.

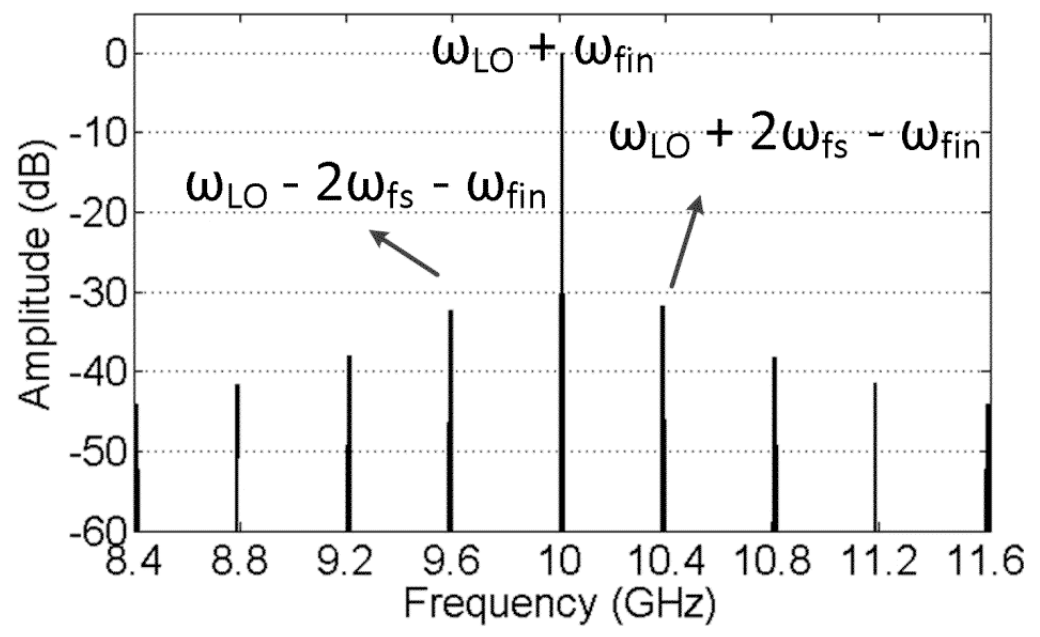

Figure 4.27. Output Spectrum of 4-path upconversion with DACs driven with multiphase sample clock and inputs delay compensated resulting in cancellation of the term at $\omega_{L O}-\omega_{f i n}$. 


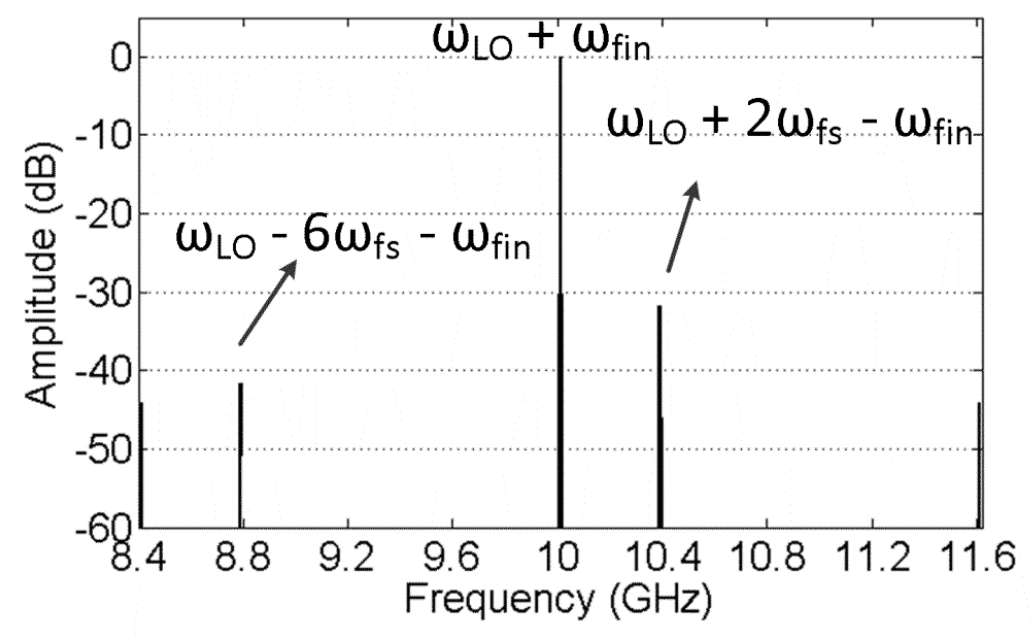

Figure 4.28. Output Spectrum of 8-path upconversion with DACs driven with multiphase sample clock and inputs are delay compensated.

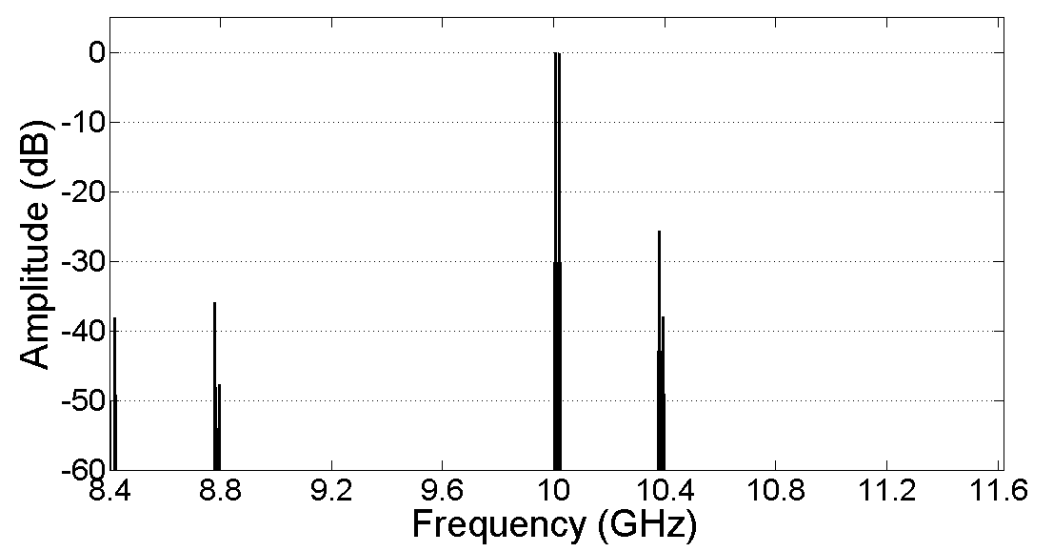

Figure 4.29. Output Spectrum of two tones ( $5 \mathrm{MHz}$ and $20 \mathrm{MHz}$ ) 8-path upconversion with DACs driven with multiphase sample clock and the two-tone inputs are delay compensated.

The conclusion we draw from the above discussion is that polyphase multipath upconversion using polyphase DAC clocks is more beneficial for cancelling DAC images compared to the case where the same clock is used for all parallel DACs. The image 
occurring at $\omega_{\mathrm{LO}+} 2 \omega_{\mathrm{fs}}-\omega_{\text {fin }}$, which is the closest most dominant image remains uncancelled due to its in-phase addition at the output, while the image of the fundamental around the LO can be cancelled if proper phase compensation is provided to the inputs.

\subsection{DAC Implementation}

In this section we discuss some implementation options of an on-chip DAC to drive the multipath mixers. Since the first set of phase shifters are in digital, the digital data for each path needs to be converted to the analog domain to drive the mixers constituting the Power Upconverter (PU). Two different possibilities to implement these DACs are discussed in the following sub sections.

\subsubsection{N parallel DACs}

One possibility is to use separate DACs running at the sample frequency of the digital data for each phase shifted data stream shown in Figure 4.30. BB0, BB1,.. are the analog baseband signals driving the multipath mixers. The architecture of Figure 4.30 could be implemented on chip, but it would make the design quite large and complex. Also maintaining good matching between the DACs would be a challenge, since each DAC would have its own reference voltage or current. Another possibility is to use one reference voltage ladder but realize separate switching networks for each set of phase shifted digital data as shown in Figure 4.31. As the voltage reference is common for all the digital-toanalog switching networks, any variation in the reference voltage would be common to all the paths. Also the distortion in each path due to reference variation would be minimized at the output as compared to the architecture in Figure 4.30, although a disadvantage would be some crosstalk due to switching noise coupling into the ladder.

The coding scheme for the switching networks could be either binary or one-hot encoded. Figure 4.32 shows a 2-bit architecture of these two different encoding schemes. In simulation we saw that an 8-bit binary coded scheme was able to achieve similar intermodulation distortion performance (for the same sample frequency and load) if the DAC switch size was made ten times bigger than the one used for the one-hot encoded switches (switch size chosen close to minimum size). This difference was mainly due to the higher number of switches in series in the path of the binary encoded architecture from the ladder to the load. As the switching networks have to be parallel eight times, so the total area would also have to be considered. Therefore just considering the switching network size, the one-hot coded scheme would be preferable. 


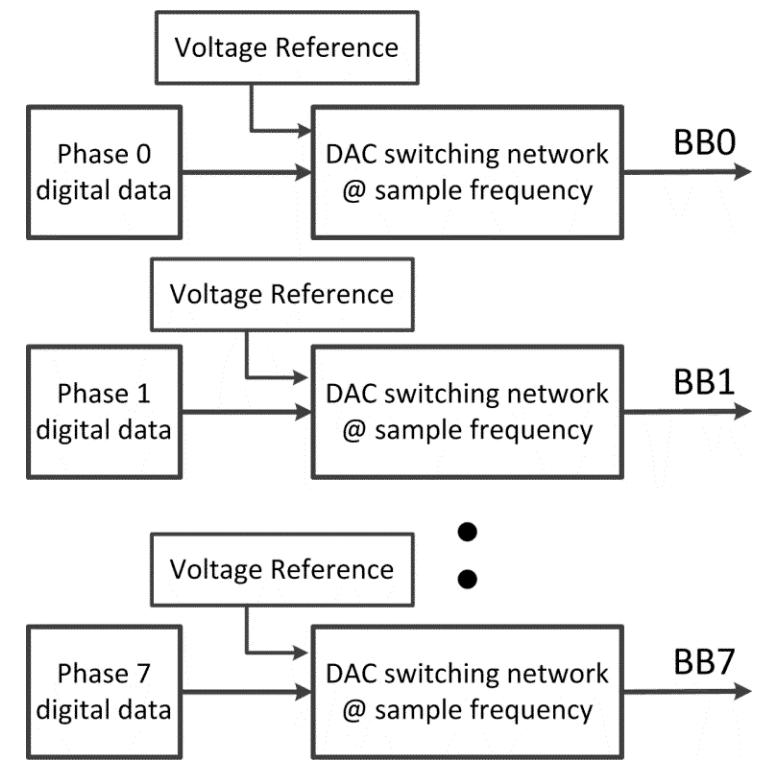

Figure 4.30 Separate Reference generation and switching network for each DAC.

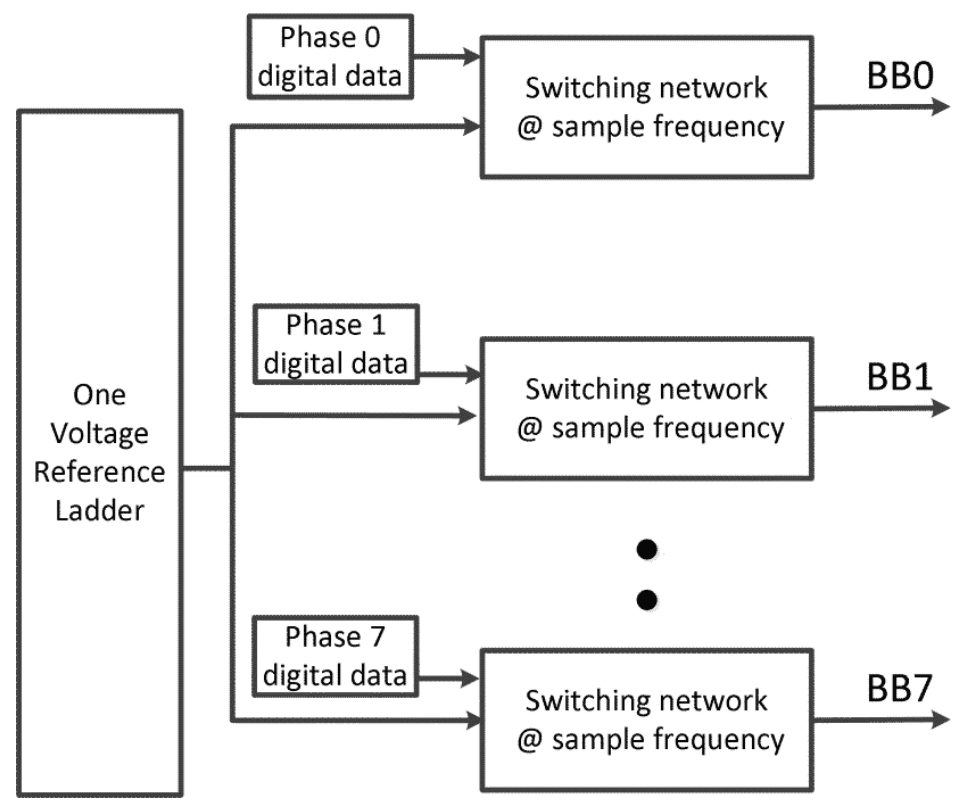

Figure 4.31 One reference ladder and separate switching networks generate the multiphase baseband, to have similar distortion in all paths as compared to Figure 4.30. 

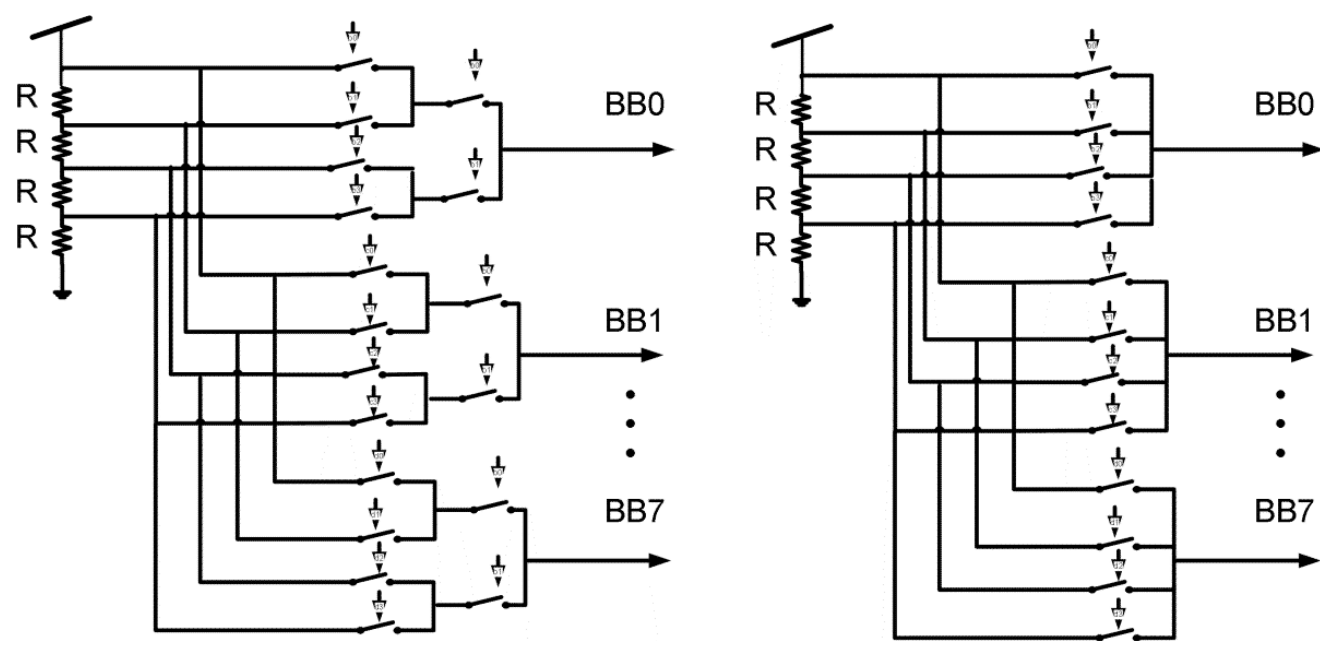

Figure 4.32. Binary (left) versus one-hot encoding (right)

\subsubsection{One DAC with de-interleaving}

Another possibility to generate the eight analog multiphase signals is to use one stand alone DAC running at $8 \mathrm{x}$ the sample rate as shown in Figure 4.33. It generates a time interleaved (multiplexed) version of all the 8 baseband paths, which are separated again by deinterleaving switches and hold functions to generate the eight analog signals $(\mathrm{BBO}$, BB1..BB7). The eight phase Polyphase clock required to drive the de-interleaving switches, can be generated by the circuit discussed in section 5.2.1. It should be noted that the $8 \mathrm{x}$ frequency required as input to such a polyphase clock generator circuit would be the same as the $8 \mathrm{x}$ sample frequency for the DAC, so an additional reference clock would not be required. In order to have increased chip testing flexibility, we can generate the digital multiphase baseband signals and use a digital to analog convertor off chip for evaluation purposes. In such a scenario only one analog input pin would be required for the chip to convey the high speed DAC output to the de-interleaving switches, which are implemented on chip. The off-chip DAC and the de-interleaving interface should preferably be impedance matched to a transmission line to minimize reflections.

The 8-phase polyphase clocks driving the de-interleaving switches can have 1/8 duty cycle as shown in Figure 4.34. This ensures that only one de-interleaving switch is tracking the input signal while the other paths are holding their last sample value. This way one path does not influence the other significantly. An implementation of the de-interleaving switches is presented in the next chapter. 


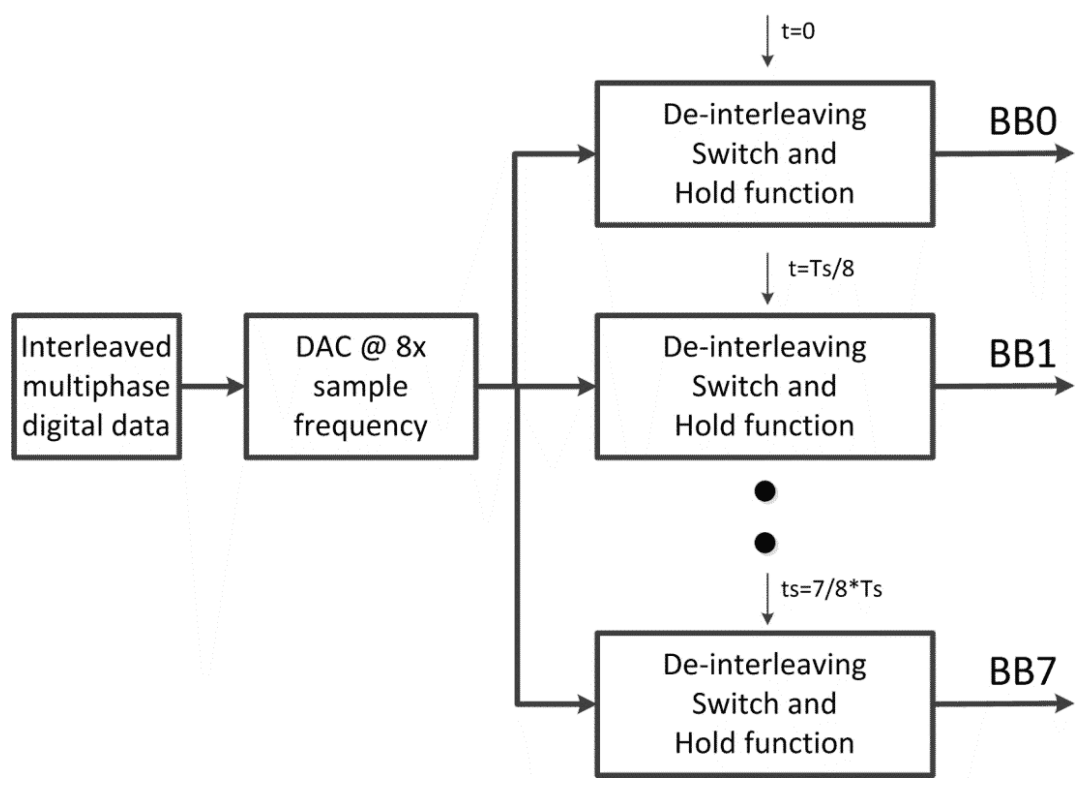

Figure 4.33 One DAC and de-interleaving

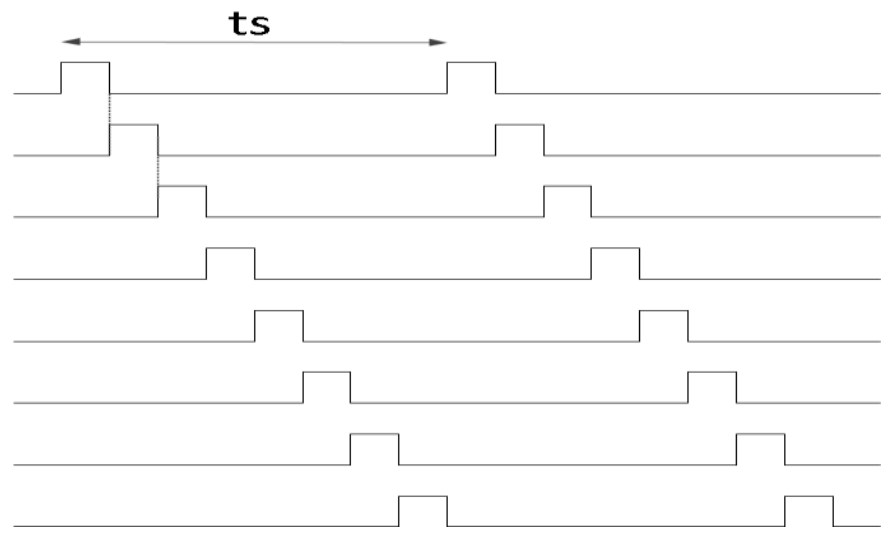

Figure 4.34. 8-phase clock driving the de-interleaving switches

\subsection{Digital Interpolation Filter}

The output of a digital to analog conversion contains many images of the input signal occurring around the sampling clock as discussed in section 4.3. These images are conventionally suppressed via an analog reconstruction filter. A digital interpolation filter allows for going to a higher sample rate (oversampling) which shifts the images to a higher 
frequency. The oversampling in combination with an analog filter relaxes the reconstruction filtering requirements. The digital filter becomes more crucial if the input to the chip has a low oversampling rate (or nyquist rate) restriction, e.g. due to DAC-bus or DAC speed limitations. If the chip interface can sustain a high sample rate and further relaxation in analog reconstruction filtering is not required, then on-chip oversampling may not be needed. For a cognitive radio transmitter an analog signal bandwidth of at least $6 \mathrm{MHz}$ is desired, which would imply $3 \mathrm{MHz}$ in both the I and Q channels. Oversampling the digital baseband using digital interpolation depends upon how much area and power can be sacrificed in the digital domain to gain the benefit of analog filter relaxation. Another criterion to consider while designing such a digital filter is the speed limits of the IC technology being used for implementation. Considering these factors and aiming for better than $50 \mathrm{~dB}$ suppression of the DAC images, an 8 times oversampled baseband $\left(\mathrm{f}_{\mathrm{s}}=48 \mathrm{MHz}\right)$ in combination with a $2^{\text {nd }}$ order analog filter can be chosen. The 8 times oversampling in combination with the zero order hold response of the DAC provides $30 \mathrm{~dB}$ suppression (from (4.8)) of the second DAC image (occurring around $2 * \mathrm{f}_{\mathrm{s}}$ ) which remains un-cancelled when upconverted as shown in Figure 4.28.

The remaining suppression can be provided by a $2^{\text {nd }}$ or higher order analog filter. This can be seen in Figure 4.35, where the sinc response (from the sinc response equation 4.8) is plotted as a function of the frequency. Also an ideal low pass $1^{\text {st }}$ order RC filter response, and a $2^{\text {nd }}$ order (cascade of two RC filters) filter response is also shown. The $2^{\text {nd }}$ order filter has a $-3 \mathrm{~dB}$ corner of $10 \mathrm{MHz}$. Adding up the suppression of the sinc and the $2^{\text {nd }}$ order filter $<-40 \mathrm{dBc}$ is achieved around $\mathrm{f}_{\mathrm{s}}(48 \mathrm{MHz})$ and $<-55 \mathrm{dBc}$ around $2 \mathrm{f}_{\mathrm{s}}(96 \mathrm{MHz})$.

\subsubsection{Digital Filter Design}

The digital interpolation filter should be linear phase so that it can provide a frequency independent delay. This would mean that it provides the same phase shift at one particular frequency in each path, so it does not impact the relative phase relationship in each path. A linear phase interpolation filter can be implemented by a direct-form FIR filter [13]. A simple 11 tap filter [13] architecture of a 2 times interpolation stage can be implemented as shown in Figure 4.36. Here the coefficients were determined by using the filter design utility fdatool in MATLAB. Here the taps 1, 3, 7 and 9 are zero (not present in Figure 4.36). Since the other coefficients are symmetric around the centre tap, the architecture can be further simplified by reducing the number of multiplications. The filter achieves better than $50 \mathrm{~dB}$ suppression above the stop-band as shown in Figure 4.37. Three such stages are cascaded in series to achieve 8 times oversampling in total. Although such a filter design can also be optimized further by having more taps in the earlier stages which work at lower frequency and hence would consume low power. Performance of an interpolation stage to 
relax analog filtering can be improved further with newer CMOS technologies. An 8x interpolation filter designed with the architecture shown in Figure 4.36 and synthesized in Cadence synthesis tool in a 160nm CMOS process consumed an area of $190 \mu \mathrm{m} \times 190 \mu \mathrm{m}$ (VHDL code in APPENDIX C). The filter worked with an output frequency of $50 \mathrm{MHz}$, with a 16-bit processing resolution and the expected power consumption is $10 \mathrm{~mW}$. The power consumption of this non-optimized design is still relatively low compared to the overall power consumption of the complete transmitter (discussed in the next chapter).

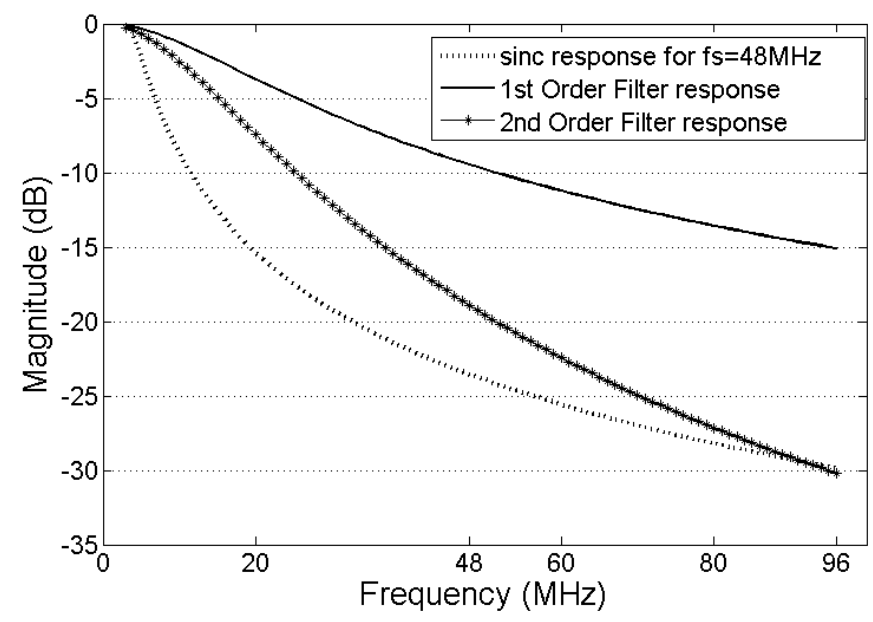

Figure 4.35. Suppression due to sinc response and an RC first order and an RC second order low pass Filter. Adding the attenuations of the sinc response and second order filter close to $96 \mathrm{MHz}$, achieves $<-55 d B$. 


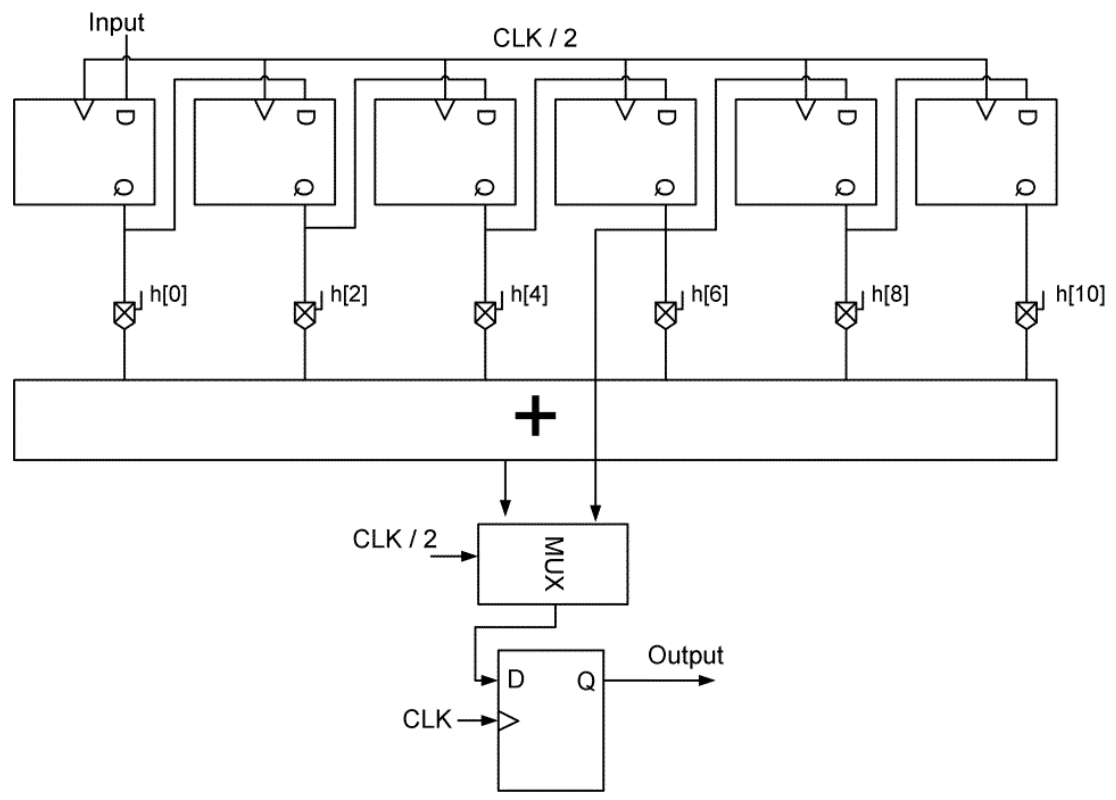

Figure 4.36 Structure for a $2 x$ interpolation filter

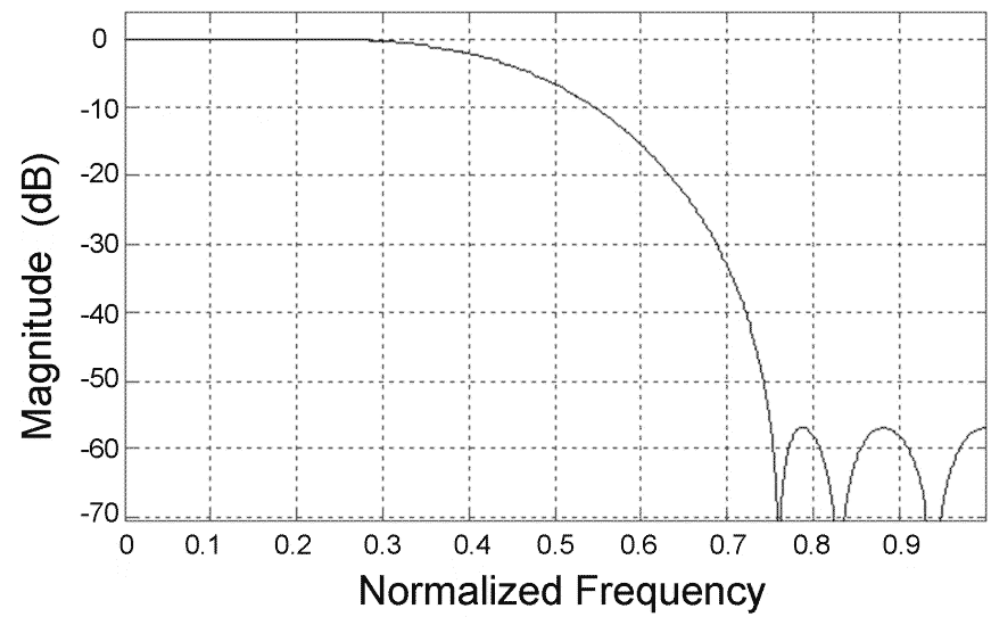

Figure 4.37 Interpolation filter response normalized to clock frequency 


\subsection{Conclusion}

An important issue in the design of a complete digital to RF polyphase multipath transmitter is the mixed signal system design. In this chapter requirements on the mixed signal system design are discussed and possible solutions are analyzed. It is shown that it is possible to generate the multiphase baseband signals required for an 8-path upconversion by using simple vector addition, employing a simplified vector modulator type of architecture. A design was synthesized in Cadence synthesis tool in 160nm CMOS running at $50 \mathrm{MHz}$ and 16 bit resolution. The estimated power consumption is only $2.5 \mathrm{~mW}$.

The multipath polyphase transmitter design can in principle cancel harmonics responses in the DAC output resulting from quantization errors. However, since inter-modulation terms remain un-cancelled so its usefulness is limited. For a single-tone and multi-tone transmit signal, at least $8 \mathrm{~dB}$ and $10 \mathrm{~dB}$ per bit SFDR can be achieved assuming the first set of phase shifters are ideal while the second set of phase shifts are provided by mixer, whose nonlinearity is modeled as a power series. Hence, in order to keep the SFDR due to limited DAC resolution better than $50 \mathrm{~dB}$ at least a 7 bit DAC resolution is required.

It is possible to cancel the first dominant DAC image by using a multiphase DAC architecture. The dominant non-cancelled image is then at $2 * \mathrm{f}_{\mathrm{s}}$, which occurs due to its almost in-phase addition in all the paths.

We can implement either a single DAC on chip, using a single voltage reference and multiple switching networks, or we could implement a single DAC and use on chip deinterleaving switches to make the 8 analog baseband signals. For testing flexibility one external DAC and on chip de interleaving was chosen.

Digital interpolation filters can relax the reconstruction filtering requirement to a second order filter with limited power overhead. A digital FIR filter design was synthesized in Cadence synthesis tool in $160 \mathrm{~nm}$ CMOS running at $50 \mathrm{MHz}$ and 16 bit resolution. The estimated power consumption is only $10 \mathrm{~mW}$.

\section{References}

[1] S. K. Garakoui, E. A. M. Klumperink, B. Nauta, and F. E. van Vliet, "Time delay circuits: A quality criterion for delay variations versus frequency," in Circuits and Systems (ISCAS), Proceedings of 2010 IEEE International Symposium on, 2010, pp. 4281-4284. 
[2] F. Behbahani, Y. Kishigami, J. Leete, and A. A. Abidi, "CMOS mixers and polyphase filters for large image rejection," Solid-State Circuits, IEEE Journal of, vol. 36, pp. 873-887, 2001.

[3] B. Razavi, RF Microelectronics. Upper Saddle River, NJ: Prentice Hall, 1998.

[4] Y. Yu, P. G. M. Baltus, and A. H. M. van Roermund, Integrated $60 \mathrm{GHz} R \mathrm{RF}$ Beamforming in CMOS: Springer, 2011.

[5] N. Blachman, "The intermodulation and distortion due to quantization of sinusoids," Acoustics, Speech and Signal Processing, IEEE Transactions on, vol. 33, pp. 1417-1426, Dec. 1985.

[6] M. J. M. Pelgrom, Analog-to-Digital Converters: Springer, 2010.

[7] M. S. Oude Alink, A. B. J. Kokkeler, E. A. M. Klumperink, K. C. Rovers, G. J. M. Smit, and B. Nauta, "Spurious-Free Dynamic Range of a Uniform Quantizer," Circuits and Systems II: Express Briefs, IEEE Transactions on, vol. 56, pp. 434 - 438, June 2009.

[8] R. Shrestha, E. A. M. Klumperink, E. Mensink, G. J. M. Wienk, and B. Nauta, "A Polyphase Multipath Technique for Software-Defined Radio Transmitters," Solid-State Circuits, IEEE Journal of, vol. 41, pp. 2681-2692, 2006.

[9] R. Gray, "Quantization noise spectra," Information Theory, IEEE Transactions on, vol. 26, pp. 1220-1244, Nov. 1990.

[10] P. T. M. van Zeijl and M. Collados, "On the Attenuation of DAC Aliases Through Multiphase Clocking," Circuits and Systems II: Express Briefs, IEEE Transactions on, vol. 56, pp. 190-194, March 2009.

[11] J. Deveugele, P. Palmers, and M. Steyaert, "Parallel-Path Digital-to-Analog Converters for Nyquist Signal Generation," Solid-State Circuits, IEEE Journal of, vol. 39, pp. 1073-1082, 2004.

[12] R. G. Lyons, Understanding Digital Signal Processing Upper Saddle River, NJ.: Prentice Hall, 2001.

[13] X. He, J. van Sinderen, and R. Rutten, "A 45nm WCDMA Transmitter Using Direct Quadrature Voltage Modulator with High Oversampling Digital Front-End," in International Solid State Circuits Conference, 2010. 


\section{CHAPTER FIVE}

\section{Polyphase Multipath Transmitter Implementation}

(This chapter is taken from the sections III-VI of the paper published in the IEEE Journal of Solid State Circuits [1]. The only differences are the addition of section 5.2 and the Figures 5.5, 5.6, 5.7, 5.16 and 5.24).

This chapter describes the implementation in $160 \mathrm{~nm}$ CMOS of the 8-path polyphase multipath transmitter. For flexibility reasons an off chip DAC was utilized. Each of the 8paths in Figure 5.1 consist of a sample and hold block (discussed previously in section 4.4.2), buffer and baseband filter (mentioned previously in section 4.5) and a switched transconductor upconversion mixer. The current output of the mixer blocks is combined to drive the antenna load. A tunable low-pass filter at the output (discussed previously in section 3.2.1) provides filtering over a wide band. A replica bias circuit is used to stabilize the bias current of the mixers over Process-Voltage-Temperature (PVT) variations, attempting to keep the output power stable. An 8-phase LO with 1/8 duty-cycle drives the eight sample and hold ( $\mathrm{S} \& \mathrm{H})$ switches (discussed previously in section 4.4.2). Another 8phase LO with $\sim 7 / 16$ duty-cycle (discussed previously in section 3.4 ) drives the eight up conversion mixer switches.

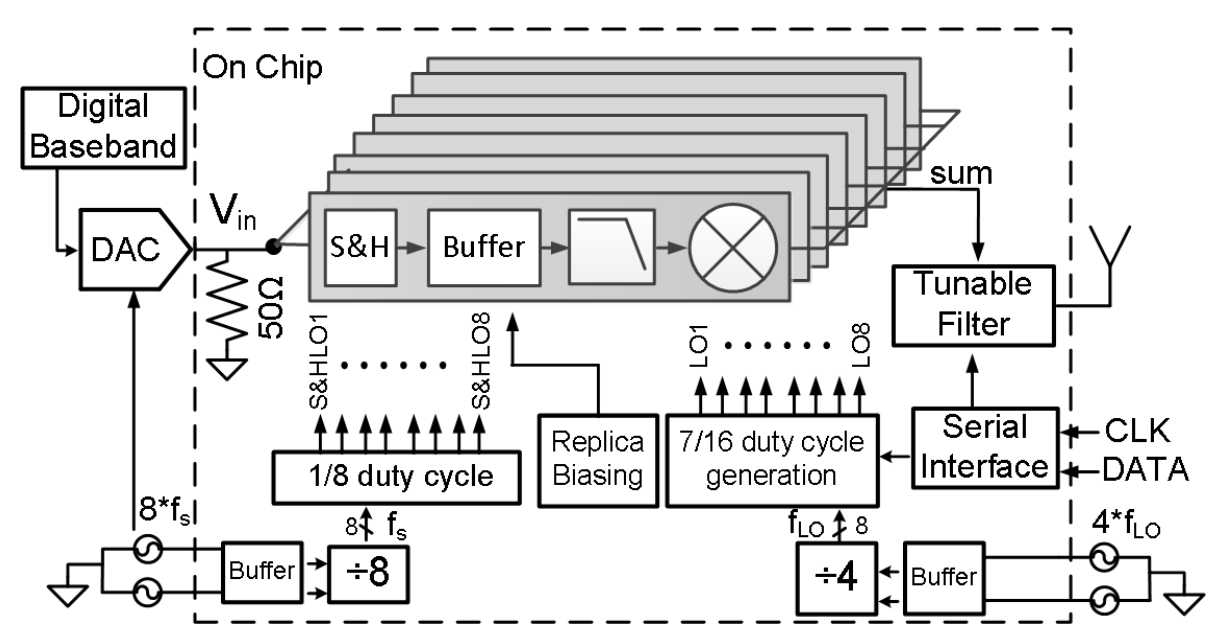

Figure 5.1 Block Diagram of the implemented 8-path Transmitter. 
The circuit implementation did not aim for any specific standard but rather explored what can be achieved with this technique in practice, especially compared to [2]. Circuit details for these blocks are given in the following sections.

\subsection{Baseband Generation}

Figure 5.2 shows the circuit present in each baseband and upconversion path, driven by an (external) DAC. The use of one DAC for all 8-paths is preferable to maximize matching of the signal paths. The 8 polyphase baseband signals are generated digitally and drive the $\mathrm{DAC}$ in a time interleaved way at 8 times the baseband sampling frequency. The sample switch $\mathrm{S} 1$ in the sample and hold $(\mathrm{S} \& \mathrm{H})$ of each path de-interleaves the DAC signal into 8 parallel analog baseband signals. To this end, the S\&H switches are driven by an 8-phase non-overlapping 1/8 duty-cycle clock (S\&HLO).

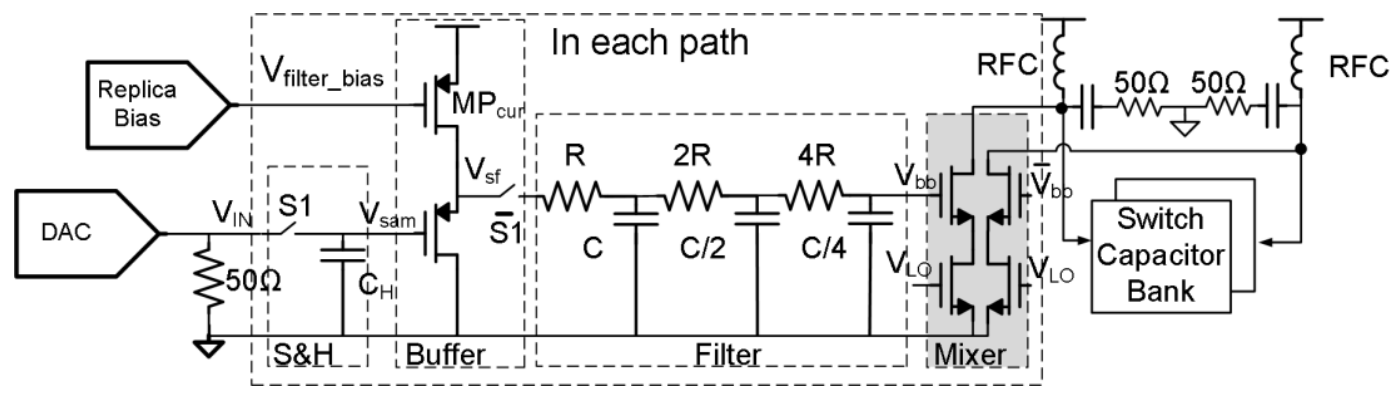

Figure 5.2 One Baseband to RF path with $S \& H$, filter and mixer.

The S\&H is designed such that it is not limiting the linearity of the upconverter at full swing; also the switch size is small which does not require significant power consumption. The interleaving mechanism can be understood as the generation of a high frequency signal, while the de-interleaving could be understood as the down-conversion of a high frequency signal to baseband along with the generation of the required phases. Figure 5.3 shows an ideal time domain output of the DAC for a single tone polyphase baseband. Two of the de-interleaved baseband signals are also shown for visualization. The buffer provides isolation between the S\&Hs and the baseband filters. Isolation during the on-time of the $\mathrm{S} \& \mathrm{H}$ is further improved by the switch $\overline{S 1}$, which disconnects the baseband RC filter from the $\mathrm{S} \& \mathrm{H}$ during the $\mathrm{S} \& \mathrm{H}$ switching transients [3]. For a channel bandwidth of $6 \mathrm{MHz}$ ( $3 \mathrm{MHz}$ in both I and Q), the sampling frequency $\left(\mathrm{f}_{\mathrm{s}}\right)$ was set at $48 \mathrm{MHz}$. 


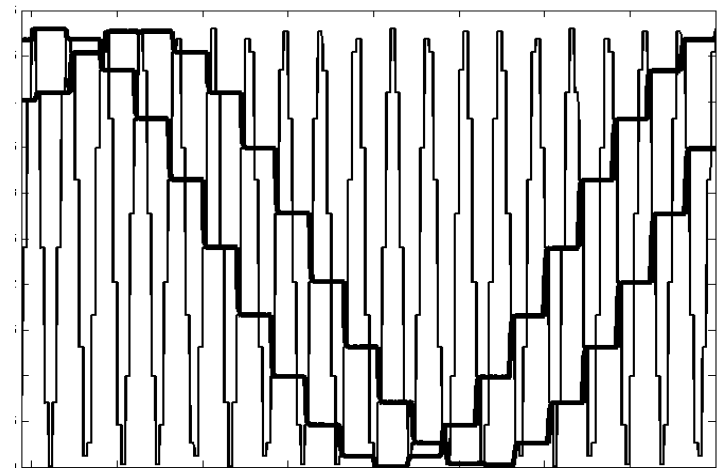

Figure 5.3 DAC output containing all the 8 polyphase baseband signals (2 of the phases are in bold).

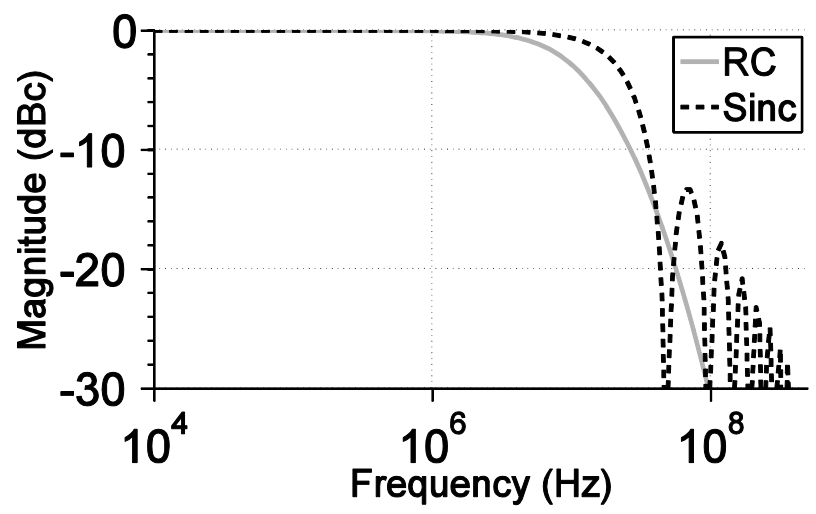

Figure 5.4 Sinc and RC low-pass filtering, achieving $<-55 d B c$ at $2 \times f_{s .}$.

The sinc response due to the DAC hold function and the baseband filter response are shown in Figure 5.4. The multiphase baseband when upconverted via the multiphase mixers results in cancellation of the first DAC replica image (see section 4.3.2 (case 2) and [4]) occurring around $\mathrm{f}_{\mathrm{s}}$. The second DAC replica image around $2 \mathrm{f}_{\mathrm{s}}=96 \mathrm{MHz}$ is suppressed to $<-55 \mathrm{dBc}$ due to the combination of $\mathrm{RC}$ filter and the sinc response as shown in Figure 5.4 (adding the attenuations). A cascade of three RC sections with increasing resistance but with equal pole frequency of $10 \mathrm{MHz}$ was used as low-pass filter. A replica bias circuit (discussed in section 5.4) controls the bias current of the mixer by adjusting $V_{b b}$. 


\subsection{8-phase LO generation}

Theoretically, a 7/16 duty-cycle [5] mixing clock can be realized by dividing a 16x higher clock. Compared to a DLL, this is more power efficient for the same mismatch accuracy [6], but there is a limit to the maximum frequency which can be achieved. In the $160 \mathrm{~nm}$ CMOS technology that we used, $16 x 800 \mathrm{MHz}$ was problematic.

Since 0.4365 is rather close to 0.50 , we use an 8 -phase $50 \%$ duty-cycle clock generated by a divide-by-4 circuit and delay the rising edge (only by a ratio of 0.063 ) to reduce dutycycle. The buffer afterwards again makes the edge steep. The 8-phase 50\% duty cycle clock is generated by a divide by four circuit, where both outputs of the flip flop are used to get all the 8 phases shown in Figure 5.5. The flip flops are made using Transmission gate (TG) logic, which provides better power and mismatch performance as compared to some other logic families [7, 8]. A high frequency sine wave is converted to a differential signal via an external balun. It is converted into a square wave via the on chip self biased inverter buffers shown in Figure 5.6. Two on chip $50 \Omega$ resistors provide the matching.

In circuit simulation, the divide by 4 circuit was able to function properly upto an LO frequency of $1.2 \mathrm{GHz}$ equivalent to $4.8 \mathrm{GHz}$ input frequency for the slowest corner of the 160nm CMOS process model library.

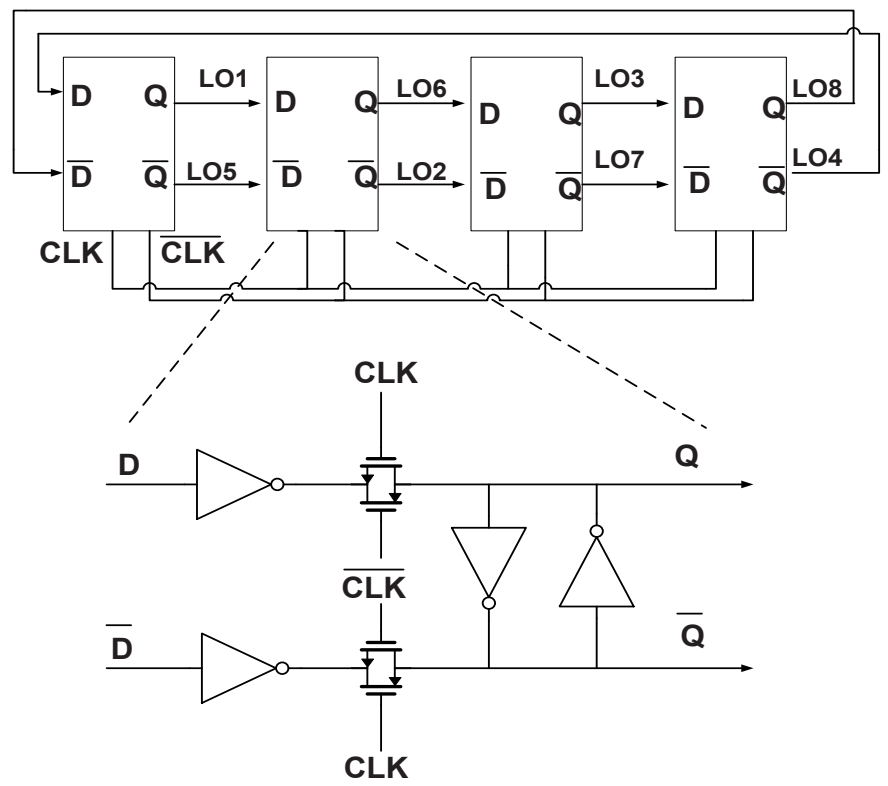

Figure 5.5 Divide by 4 implemented as a chain of flip flops 


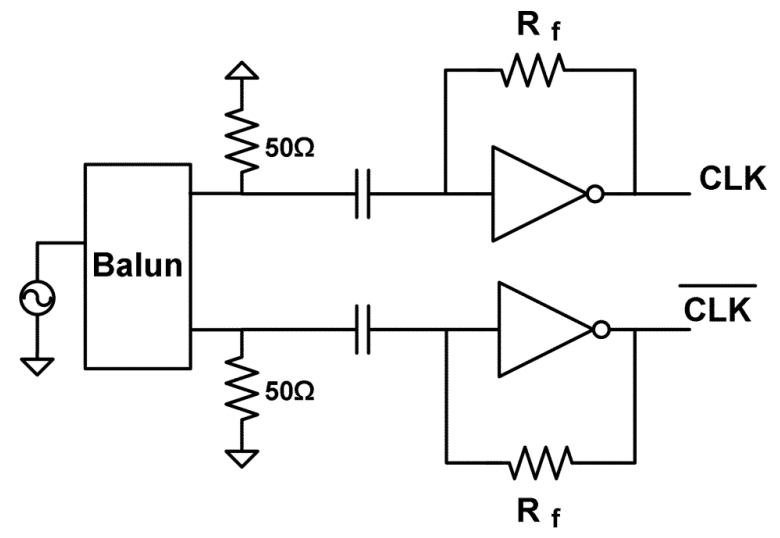

Figure 5.6 Sine wave input converted to a differential square wave on chip.

\subsubsection{Generating $1 / 8$ duty cycle for sample $\&$ hold switch}

The divide by 4 circuit is used not only for the LO path but a copy of the same circuit is utilized to generate the multiphase clock for the baseband sample and hold circuit. Since the DAC is switching at 8 times the sampling frequency, a divide-by-eight circuit is required which divides the DA clock to the sample rate needed by the sample and hold switches. Therefore a divide by two circuit was added before the divide by four as shown in Figure 5.7. To make the $1 / 8$ duty cycle, AND gates are used which combine two of the phases also shown in Figure 5.7. To avoid overlap between the clocks, the rise and fall times were adjusted at the output inverter stage inside the AND gate. This was done by making the NMOS of the inverter stronger than the PMOS, so that the fall time is faster than the rise time, allowing the crossover point of the adjacent clock phases to be low.
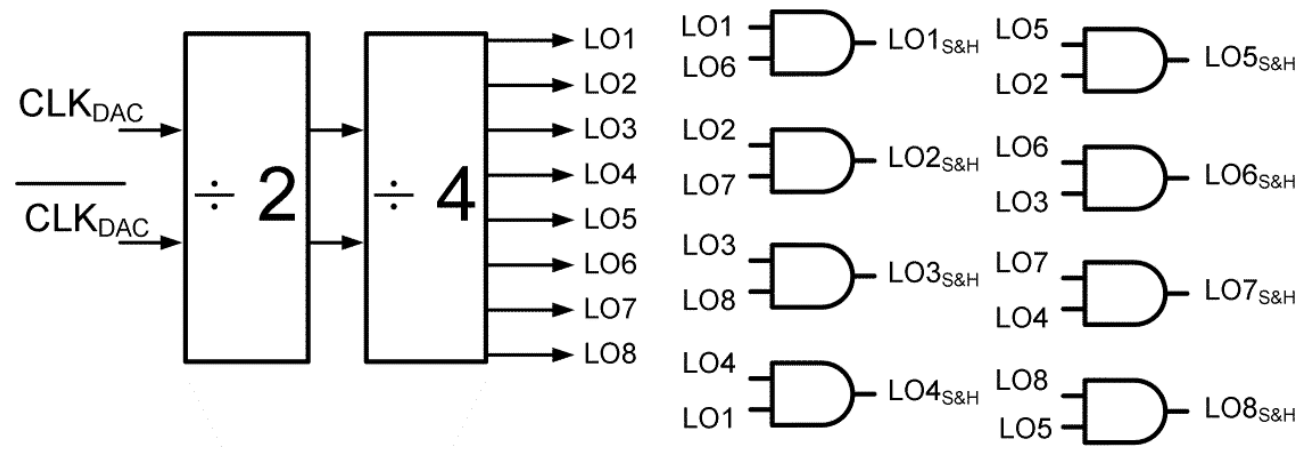

Figure 5.7. Logic to convert $50 \%$ duty cycle to $1 / 8$ duty cycle 


\subsection{Duty-Cycle Control Circuit Design}

Figure 5.8 shows how the controllable duty-cycle is realized on circuit level, by delaying the rising edge of a 50\% clock. The clock divider output is buffered and then an inverter with controllable rising edge delay drives the last buffer stage. The control is accomplished via a tunable resistor, implemented as a triode PMOS transistor (MP2). For control purposes, monotonic control is desired, which is realized by controlling the gate voltage of the triode transistors by an intrinsically monotonic resistor ladder DAC. In order to achieve this control over multi-octaves of frequency range, additional triode transistors can be added via switches $\mathrm{S}_{1}-\mathrm{S}_{5}$ as shown in Figure 5.9, where DAC voltage $\left(\mathrm{V}_{\mathrm{DAC}}\right)$ is shared. There are two main requirements on the design: 1) there should be sufficient resolution to keep the duty-cycling variations within $0.1 \%$ as discussed in section 3.2. 2) The required duty-cycle should be provided over multiple octaves of frequency range. The following sub-sections describe how these requirements can be met.

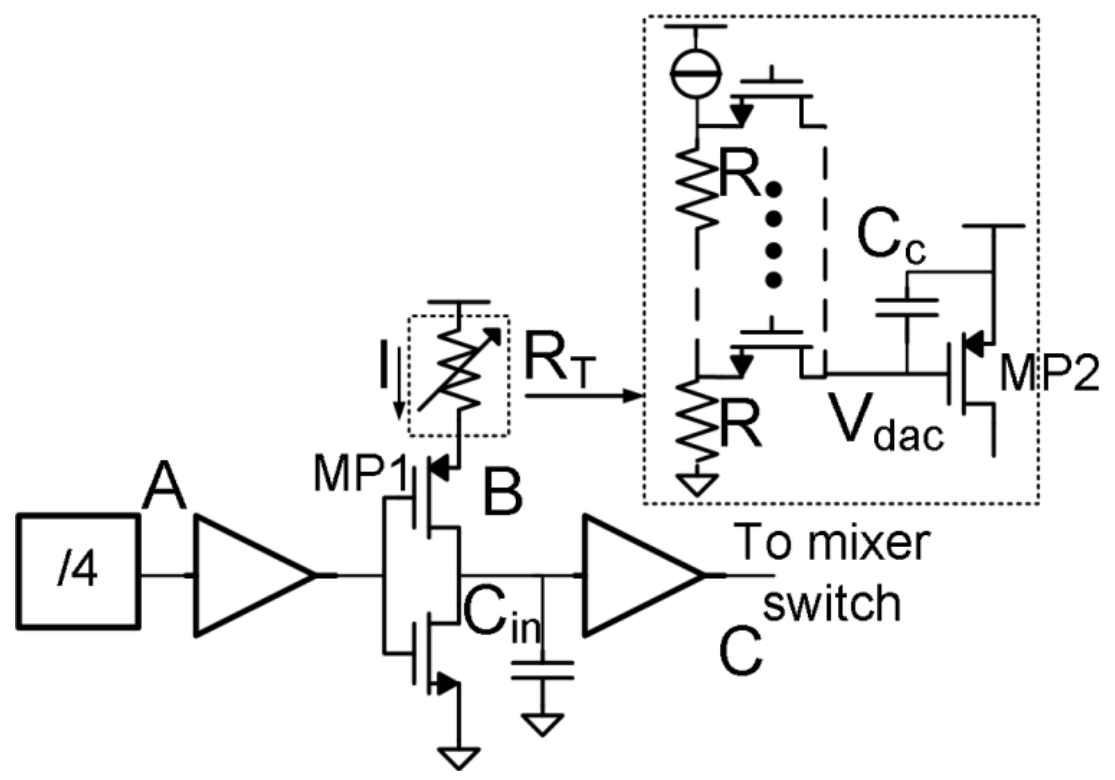

Figure 5.8. Duty-cycle control varying $R_{T}$, implemented as a triode PMOS MP2 driven by a ladder DAC. 




Figure 5.9. 7/16 LO-duty-Cycle Generation and Control over frequency.

\subsubsection{Duty-Cycle for the required accuracy}

In order to estimate the delay caused by the inverter-PMOS and triode-PMOS combination, the charge current for capacitor $C_{\text {in }}$ in Figure 5.8 is calculated. The current through the PMOS transistor MP1 degenerated by a resistance $\mathrm{R}_{\mathrm{T}}$ can be coarsely modeled as:

$$
I=\frac{\beta}{2}\left(V_{I N}-I \cdot R_{T}-V_{T H}\right)^{2}
$$

Here $\mathrm{R}_{\mathrm{T}}$ is the resistance of the triode transistor as given by (5.2) and $\beta=\mu_{p} C_{o x} \cdot \frac{W}{L}$.

$$
R_{T}=\frac{1}{\beta_{T} \cdot\left(V_{g s_{M P 2}}-V_{T H}\right)}
$$

Expanding (5.1) results in (5.3).

$$
\frac{\beta}{2}\left(I^{2} R_{T}{ }^{2}-I\left(2 \cdot R_{T} \cdot V_{I N 0}+\frac{2}{\beta}\right)+V_{I N 0}{ }^{2}\right)=0
$$




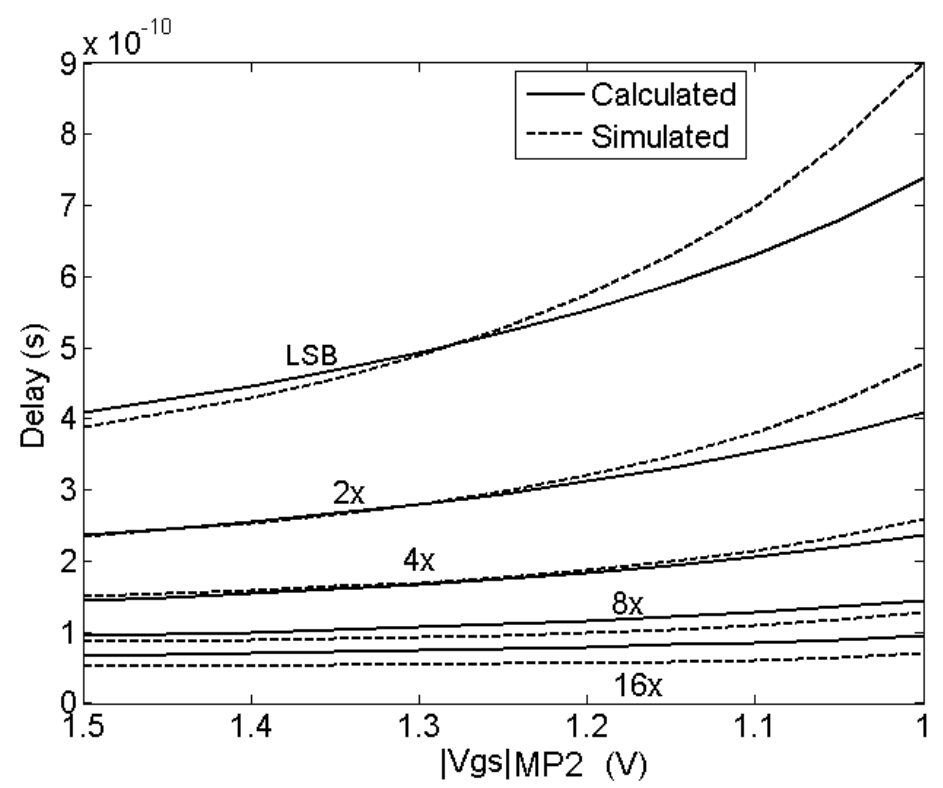

Figure 5.10 Calculated and Simulated Delay.

Where $V_{\mathbb{I N} 0}=V_{\mathbb{I N}^{-}} V_{T H}$, Solving the quadratic equation of (5.3) results in (5.4).

$$
I=\frac{V_{I N 0}}{R_{T}}+\frac{1}{\beta \cdot R_{T}{ }^{2}} \cdot\left(1-\sqrt{1+2 \cdot V_{I N 0} \cdot \beta \cdot R_{T}}\right)
$$

This current can be used to estimate the delay due to the triode PMOS by the following equation.

$\mathrm{t}_{\mathrm{d}}=\mathrm{C}_{\text {in }} \Delta \mathrm{V} / \mathrm{I}$

where $t_{d}$ is the rise time delay, $C_{i n}$ is the capacitance at the input of the last buffer and $\Delta V$ is taken as $\mathrm{V}_{\mathrm{DD}} / 2$, the point where the last buffer switches. Equation (5.5) is plotted in Figure 5.10 , after substituting the values of $C_{\text {in }}=300 \mathrm{fF}, \beta=12.5 \mathrm{~mA} / \mathrm{V}^{2}, \mathrm{~V}_{\mathrm{IN}}=1.5, \mathrm{~V}_{\mathrm{TH}}=0.5$ and $\beta_{\mathrm{T}}$ is $0.78 \mathrm{~mA} / \mathrm{V}^{2}$ for the LSB triode PMOS (MP2). Figure 5.10 also shows the simulated (in $160 \mathrm{~nm}$ CMOS process) values of the delay plotted as a function of $\mathrm{V}_{\mathrm{gs}}$ of the triode (MP2) transistor. The delays for the 2, 4, 8 and 16 times the LSB triode PMOS size are also shown. As $|\mathrm{Vgs}|$ for MP2 decreases from $1.5 \mathrm{~V}$ to $1 \mathrm{~V}$, the delay becomes more than half of its initial value. A higher delay can be achieved by reducing the $|\mathrm{Vgs}|$ further, but larger $|\mathrm{Vgs}|$ is better to keep the variation in the resistance of MP2 limited due to $\mathrm{V}_{\text {th }}$ mismatch. Also larger $|\mathrm{Vgs}|$ keeps the MP2 PMOS in deep triode. The steepness of the delay curve is lower at larger $|\mathrm{Vgs}|$, which is desired since it provides smaller delay steps. Therefore the 
range of $\mathrm{Vgs}$ chosen is from $-1.5 \mathrm{~V}$ to $-1 \mathrm{~V}$. The gate voltage required is generated by a 5-bit resistor ladder DAC which achieves a resolution of $1.25 \mathrm{ps}$ (for $800 \mathrm{MHz} \mathrm{LO}$ ) with some margin (from (5.5)). High frequency VDD variations are coupled to the gate of the triode transistors with the capacitor $\mathrm{Cc}$, attempting to leave $\mathrm{V}_{\mathrm{gs}}$ unaffected.

\subsubsection{Duty-Cycle over a Frequency Range}

In order to achieve the required duty-cycle over the 3 octaves frequency range an estimate is needed of the resistance value of the tunable resistor. This resistance value should satisfy the boundary conditions of achieving the required delay over the frequency range. The total rising edge delay required is about $1 / 8$ of the LO time period. As there will always be some fall time present in the LO path, that has to be taken into consideration as well. Also, depending on the exact shape of the transition edges, some fine tuning may be needed. From simulations done on the LO path, the falling edge delay time at the mixer switch remains around 70-90ps for the 100-800- $\mathrm{MHz}$ frequency range. After subtracting the fall time from the required rising edge delay time, an estimate of the rising edge delay $t_{d}$ is found. This delay is in the range of $60 \mathrm{ps}$ to $1.1 \mathrm{~ns}$ (for the highest and lowest frequency). The resistor values can be estimated by substituting (5.4) in (5.5) and solving the quadratic equation for $\mathrm{R}_{\mathrm{T}}$.

$$
R_{T}=\frac{t_{d}}{V_{D D} \cdot C_{i n}} \cdot\left(V_{I N 0}+\sqrt{\frac{2 \cdot V_{D D} \cdot C_{i n}}{\beta \cdot t_{d}}}\right)
$$

Solving (5.6) we find that the biggest resistor required is almost 13 times bigger than the smallest value. A 5-bit resolution of the parallel triode transistor was chosen to be able to cover the frequency range with some overlap between switching of the binary weighted PMOS transistors. The switch sizes S1..S5 in Figure 5.9 were made big enough so as to not be dominanting the control mechanism. Since the DAC voltage is distributed to all the paths with some series resistance, a local decoupling capacitor is used to bypass the high frequency voltage components that can couple from the LO path to the DAC output.

\subsubsection{Duty-Cycle variation with Temperature}

In order to assess the behavior of the duty cycle control circuit with temperature variation, circuit simulations were performed on the circuit Figure 5.9 and the duty cycle was measured at the input of the mixer switch (node C). The temperature was swept from $-30^{\circ} \mathrm{C}$ to $80^{\circ} \mathrm{C}$, while the optimum code was set for $20^{\circ} \mathrm{C}$. The results in Figure 5.11 show that for around $25^{\circ} \mathrm{C}$ increase in temperature the duty cycle varies by $0.1 \%$. Further simulations 
including the mixer reveal that there is a variation in harmonic responses of about $1 \mathrm{~dB}$ from the optimum code if the temperature varies by $\pm 10^{\circ} \mathrm{C}\left(20^{\circ} \mathrm{C}\right.$ in total). So we would recommend doing an offline re-calibration for each 20 degree variation in temperature, as real time calibration during operation might cause harmful interference. Frequent recalibrations are probably not needed once chip temperature is stable within about 20 degrees.



Figure 5.11. Duty Cycle Variation with Temperature.

\subsection{Replica Biasing}

A replica biasing circuit was utilized as shown in Figure 5.12. The aim was to keep the mixer current and hence delivered power to the load controlled by a bias current. To this end a scaled replica of the switched $g_{m}$ mixer is biased by current $\mathrm{I}_{\text {bias. }}$. A diode-connected scaled $g_{m}$ transistor absorbs the current and generates a voltage $V_{\text {ref. }}$ The gate of the lower transistor in the mixer replica is connected to VDD to mimic the ON state of the mixer switch. Now $\mathrm{V}_{\text {ref }}$ is the wanted DC bias voltage corresponding to wanted mixer bias point. This voltage should be generated by all buffers if their input is equal to $\mathrm{V}_{\text {DAC_DC}}$, the middle of DAC range (externally generated here by a voltage source as the DAC was external). To do this, a buffer replica is connected to $\mathrm{V}_{\text {DAC_DC }}$ and an error amplifier adapts the gate of the PMOS current source MPcur. The amplifier is implemented by a two stage op-amp. Its high gain forces voltage $V_{\text {track }}$ equal to $V_{\text {ref }}$ by adjusting voltage $V_{\text {filter_bias, }}$ making the mixer bias insensitive for PVT variations. The $\mathrm{V}_{\text {filter_bias }}$ voltage is used as bias for all the baseband paths. The compensation capacitor $\mathrm{C}_{\mathrm{c}}$ makes sure that the phase margin of the loop is more than 70 degrees over all process corners. 


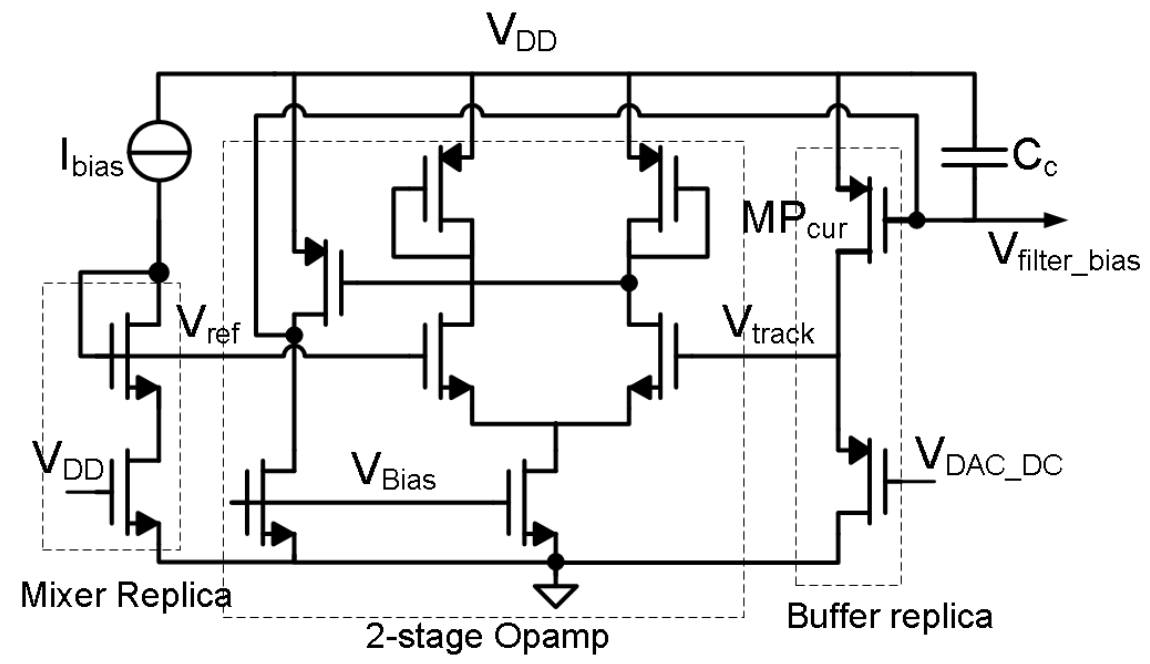

Figure 5.12 Replica Biasing Circuit adjusting $V_{\text {filter_bias. }}$

\subsection{Mixers}

The switched transconductor (switched- $\mathrm{g}_{\mathrm{m}}$ ) mixer architecture in which a transconductor is switched on and off [9] was utilized to provide frequency translation as well as power gain. Figure 5.13a shows the switched- $\mathrm{g}_{\mathrm{m}}$ mixer used in [2], where the lower transistors implement the switches and the upper ones the transconductor. In the current design, a split-switch architecture for the switched-gm mixer was utilized as shown in Figure 5.13b, where $\mathrm{Vo}+$ and Vo- are the differential outputs across the load $(2 \times 50 \Omega)$.

The switch size was divided equally between the two arms of the $g_{m}$, with negligible effect on the LO-buffer loading. This was done to shift the compression point to a higher input swing and to improve linearity. The $\mathrm{ON}$ resistance of the mixer switch now acts as source degeneration for the transconductor. As discussed in $[2,10]$ the optimum size of $\mathrm{g}_{\mathrm{m}}$ transistors and switch transistors is equal for maximum amplification for a given area. However, reducing the size of the switch is beneficial for reducing the power consumption in the driver buffers.

For the current design a size of $100 \mu \mathrm{m} / 0.16 \mu \mathrm{m}$ was chosen for each switch transistor and $200 \mu \mathrm{m} / 0.16 \mu \mathrm{m}$ for each trans-conductor. Simulations show that using the split-switch mixer (Figure 5.13b) improves the compression point and OIP3 by $3 \mathrm{~dB}$ and $1 \mathrm{~dB}$ respectively as compared to the shared-switch mixer (Figure 5.13a). 


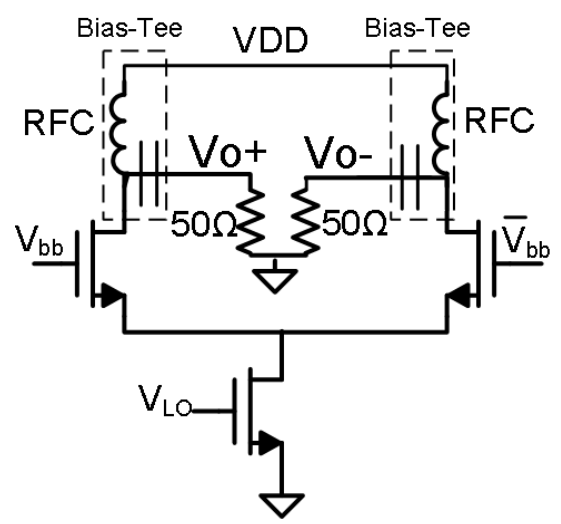

(a)

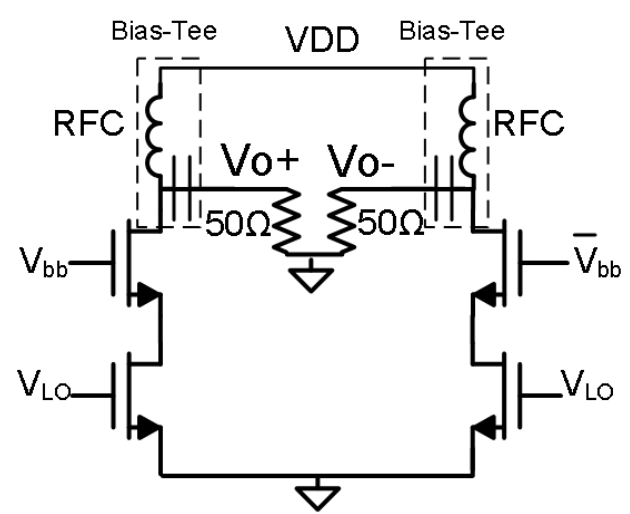

(b)

Figure 5.13 (a) Mixer used in [2] (b) Mixer in current design

\subsection{Tunable 1st Order Low-Pass Filter}

A tunable RC low-pass filter is applied at the output of the mixer to suppress the higher harmonics. A bank of switchable binary weighted capacitors in combination with the load resistor $(50 \Omega$, single ended) (shown in Figure 5.14) provide a first order RC roll off, where the current source represents the switched-g $\mathrm{g}_{\mathrm{m}}$ mixers.



Figure 5.14 Switch Capacitor bank providing output filter tuning.

The NMOS switch sizes were kept large enough to keep their ON resistance low, so as not to limit the suppression achieved. The magnitude of the fundamental component can vary significantly with LO frequency if there is not enough resolution in the capacitance bank. In order to keep these variations $<0.5 \mathrm{~dB}$ from the nominal value, a 5 bit resolution was chosen. Alternatively a coil can be added to realize a band-pass filter with tunable center frequency. This can give more suppression but results in less tuning range for the same 
capacitor variation. Since this filtering occurs after the mixers, any nonlinearity added by this filter would not be cancelled. Therefore fringe capacitors were utilized, which are more linear than MOS capacitors.

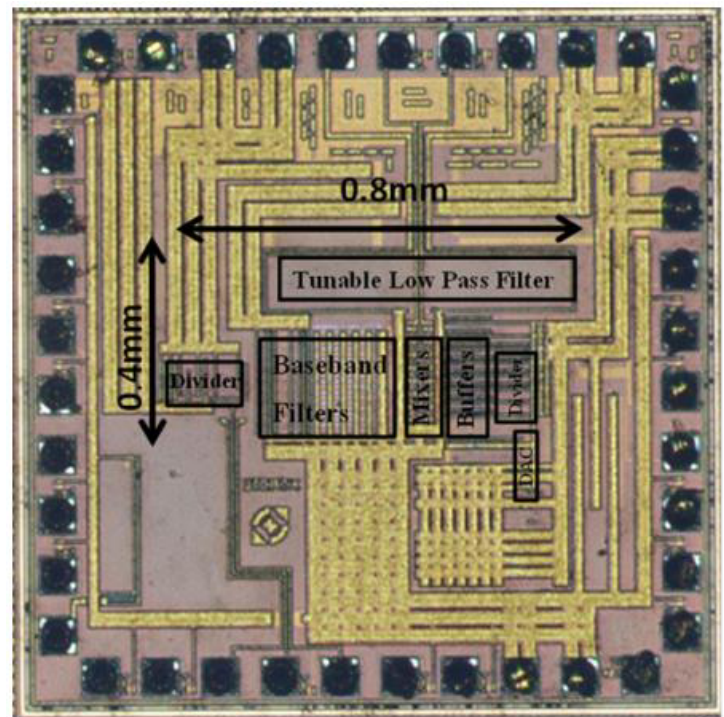

Figure 5.15 Chip micrograph with active area $<0.32 \mathrm{~mm}^{2}$ in $160 \mathrm{~nm}$ CMOS

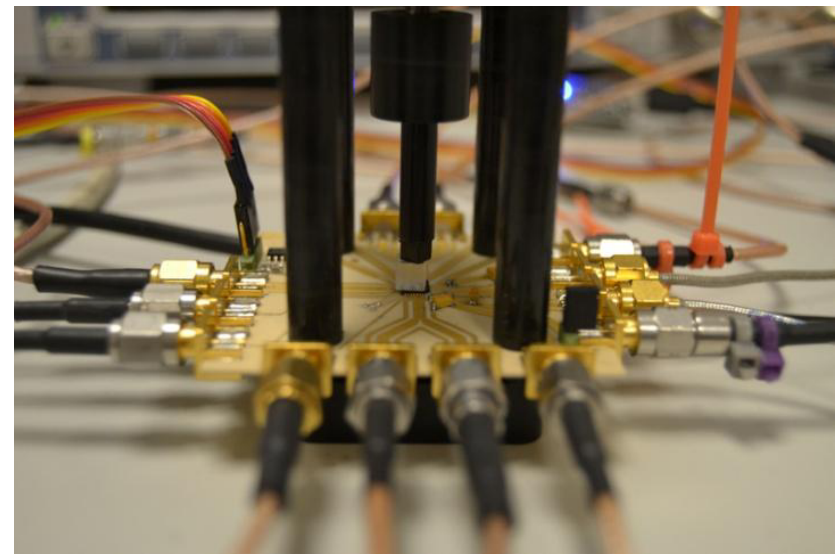

Figure 5.16. Chip Measurement Setup 


\subsection{Measurement Results}

The transmitter was implemented in a $160 \mathrm{~nm}$ CMOS process, and its die photo and measurement setup is shown in Figure 5.15 and Figure 5.16 respectively. The RF choke (see Figure 5.2) and the load $(2 \times 50 \Omega)$ are off chip. Figure 5.17 shows the measured fundamental power at $400 \mathrm{MHz} \mathrm{LO}$, as well as the $7^{\text {th }}$ and $9^{\text {th }}$ harmonic response for all the 1024 ( $5+5$ bits) duty-cycle codes. Here the code 0 implies a duty-cycle ratio close to 0.5 $\left(\mathrm{S}_{1}-\mathrm{S}_{5}\right.$ in Figure 5.9 all $\left.\mathrm{ON}\right)$ and higher codes gradually move towards lower duty-cycle ratios as explained below. The abrupt jumps in the harmonic response occur when a binary scaled PMOS transistor is turned ON/OFF by S1-S5. The smaller steps occur when selecting a next tap of the ladder DAC, which increases the $\mathrm{V}_{\mathrm{DAC}}$ increasing $\mathrm{R}_{\mathrm{T}}$ and hence decreasing the duty-cycle. Clearly, there are more than enough codes to fine tune the harmonic power with better than $1 \mathrm{~dB}$ precision via the ladder DAC (see inset of Figure 5.17).

This works uptill the maximum LO frequency of $800 \mathrm{MHz}$, beyond which the divider stops working. At code 512, the MSB switches OFF and due to the un-symmetric layout of the MSB PMOS, there is an upward jump in the harmonic response, which causes some repetition in the harmonic responses upto around code 700. This repetition along with the overlap in codes ( 2 or more codes giving the same harmonic response), can easily be detected and removed by a one-time calibration.

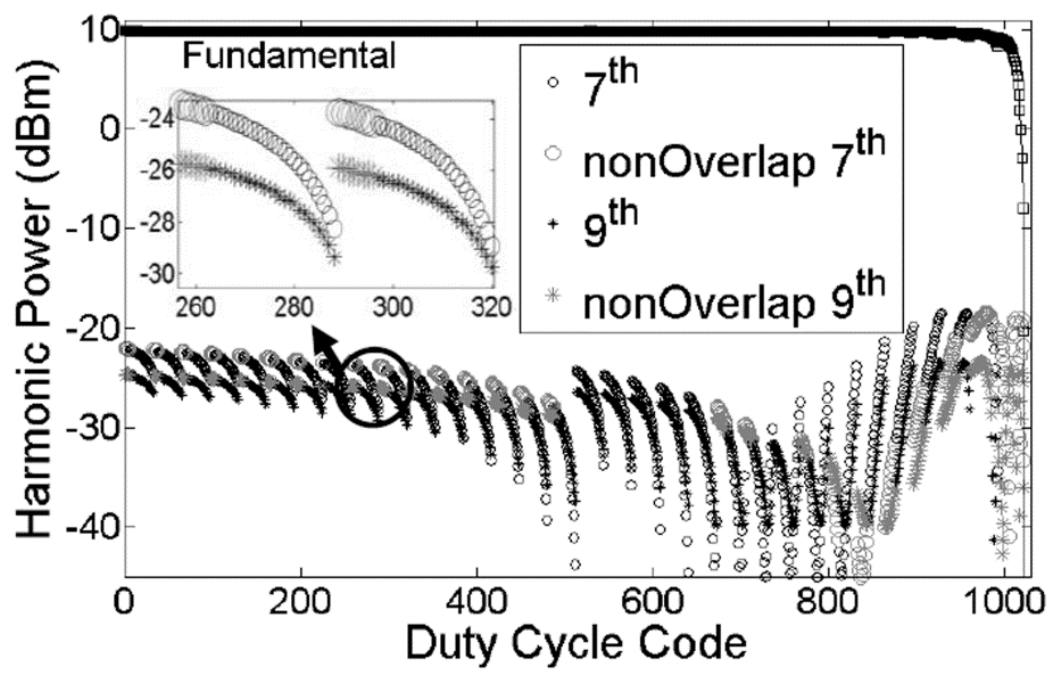

Figure 5.17. Magnitude of $7^{\text {th }}$ and $9^{\text {th }}$ harmonics vs.Duty-Cycle code(LO at 400MHz). Bigger markers are for those duty cycle codes which give non-overlapping harmonic response. 


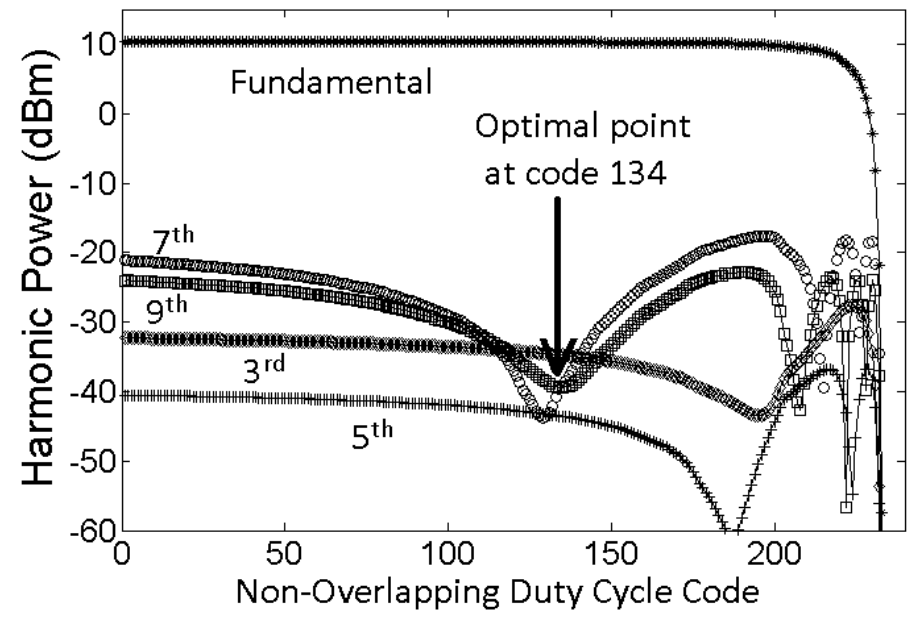

Figure 5.18. Magnitude of harmonics vs. Non-Overlapping Duty-Cycle code(LO at 400MHz). Same graph as Figure 5.17 but with overlapping harmonic responses removed.

This calibration can be done by selecting non-overlapping harmonic responses of adjacent MSB transitions as shown in the inset of Figure 5.17 (larger symbols). It should be noted here that the selected codes are the ones where the LSB switching due to the ladder DAC just begins, i.e. where $\mathrm{V}_{\mathrm{DAC}}$ is zero. This is where the triode resistance is smallest rendering lowest delay and best delay resolution, since the $\mathrm{V}_{\mathrm{gs}}$ is the maximum. After removing the redundancy a smoother curve results as seen in Figure 5.18, where the x-axis now only shows the non-overlapping codes. The figure shows the magnitude of the dominant uncancelled harmonics, occurring around the $3^{\text {rd }}, 5^{\text {th }}, 7^{\text {th }}$ and $9^{\text {th }}$ harmonics of the LO (at $400 \mathrm{MHz}$ ). In this scheme the optimum point occurs at code 134. Even after removing the redundancy there is enough resolution such that the harmonic responses to the nonoverlapping codes adjacent to the optimal point are within $0.5 \mathrm{~dB}$ of each other. The $3 \mathrm{rd}$ and 5 th LO harmonics are also better than $-44 \mathrm{dBc}$ and $-50 \mathrm{dBc}$ respectively. Measuring 10 chips from one batch (where the optimum code was extracted for 1 chip and applied to 9 other chips of the same batch ), we found this optimal point to remain within $1 \mathrm{~dB}$ and \pm 2 code steps of each other, which proves that the design has enough resolution to cover variations due to mismatch (as targeted in section 5.3.1). Similarly the mismatch measured over 10 chips of one batch for the dominant uncancelled harmonics (for the same set of nonoverlapping codes) is shown in Figure 5.19. Less than $1 \mathrm{~dB}$ variation in the $7^{\text {th }}, 9^{\text {th }}, 15^{\text {th }}$ and $17^{\text {th }}$ harmonics occurs due to their in-phase addition as was predicted by (3.18). The measurements at harmonic number -1 and 0 are for image and LO leakage respectively. 
It is also of interest to see the $5^{\text {th }}$ and $3^{\text {rd }}$ harmonic in Figure 5.18 decreasing in power around the non-overlapping codes 185 and 195 respectively, which theoretically correspond to the duty-cycle of $40 \%$ and $33 \%$ as predicted by (3.3) [5]. As the code increases the dutycycle becomes smaller and smaller, and after code 200 it becomes significantly dominated by rise and fall times, resulting in a significant drop in the fundamental output power. Figure 5.20 shows the harmonic response over LO-frequency with $5 \mathrm{MHz}$ steps, while the non-overlapping duty-cycle code was adjusted for each LO frequency by monitoring the spectrum analyzer data for the $7^{\text {th }}$ and $9^{\text {th }}$ LO harmonics and selecting the non-overlapping code where both of them are simultaneously suppressed.

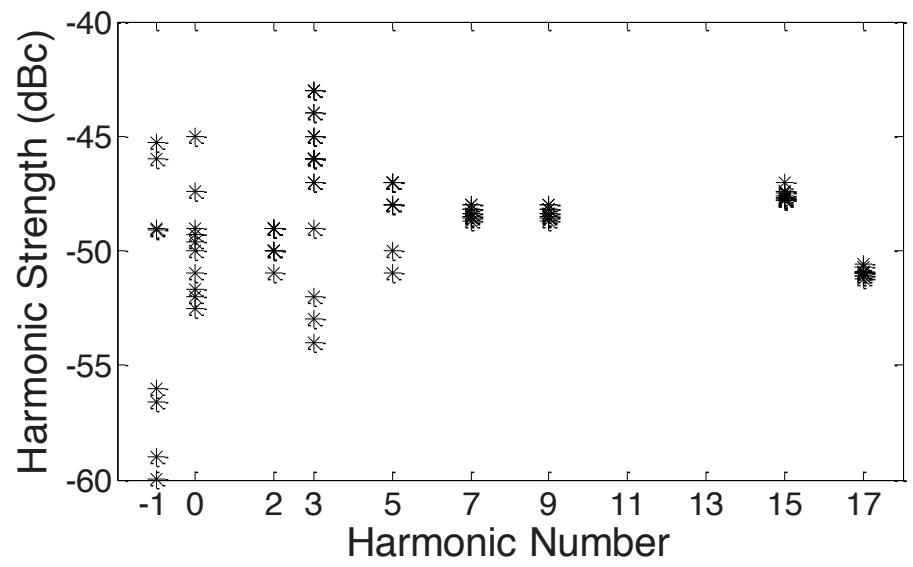

Figure 5.19 Harmonic Strength measured for 10 chips (LO at 400MHz)

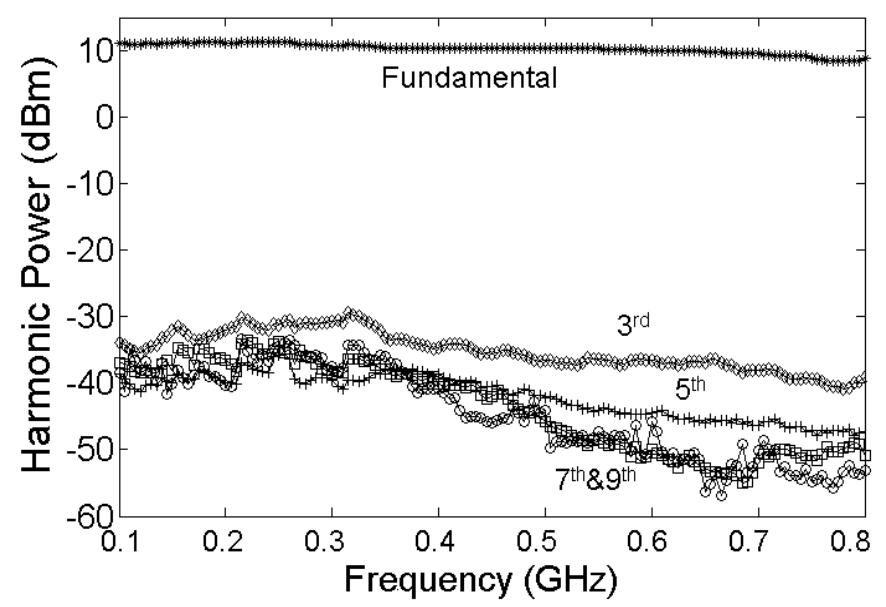

Figure 5.20. Measured magnitude of harmonics vs. LO-frequencyfor optimal code. 
The RF filter was tuned in six steps from $100 \mathrm{MHz}-250 \mathrm{MHz}$, beyond which the filter was not required, as parasitics at the output of the chip keep the 15th and 17th harmonics already below $-40 \mathrm{dBc}$.

Figure 5.21 shows the measured mixer efficiency as a function of the non-overlapping duty-cycle code when the LO is at $400 \mathrm{MHz}$. The efficiency increases as the duty-cycle code decreases and reaches a maximum around the non-overlapping code 185 which implies a duty-cycle around $40 \%$, which is close to the prediction made for the optimum by the analysis in section 3.3.

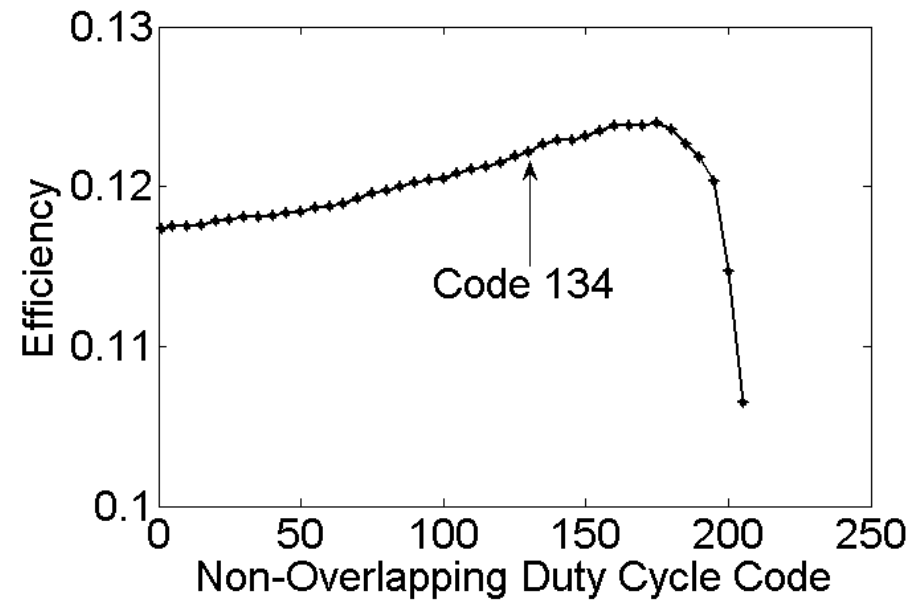

Figure 5.21 Measured Mixer Efficiency vs. Non-overlapping Duty-Cycle Code

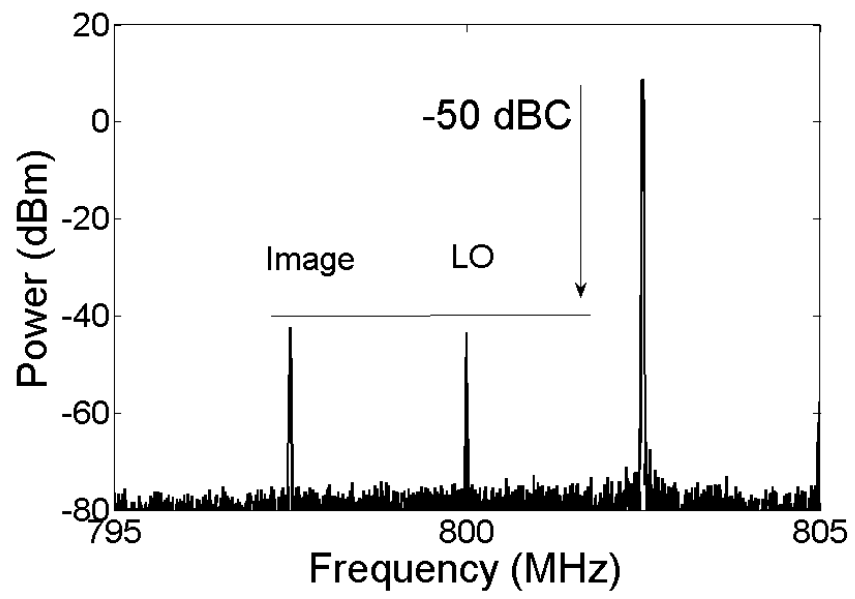

Figure 5.22 Image and LO Leakage magnitude 
Figure 5.22 shows the image and LO leakage for a $2.5 \mathrm{MHz}$ single tone upconverted by $800 \mathrm{MHz}$ to $802.5 \mathrm{MHz}$. Both are measured to be better than $-45 \mathrm{dBc}$ without calibration, over the whole band for 10 chips. This is $10 \mathrm{~dB}$ better than [2], and can be attributed to using a shared $\mathrm{DAC}$ for signal generation. The maximum single tone $\mathrm{P} 1_{\mathrm{dB}}$ output power after filtering was measured to be $+10.8 \mathrm{dBm}$, while the mixers consume $59 \mathrm{~mA}$ from a $1.5 \mathrm{~V}$ supply. The divide by 4 circuit, LO buffers and control DAC consume $33 \mathrm{~mW}$ at $500 \mathrm{MHz}$. The baseband clock divider and buffers and the baseband filters consume $13.5 \mathrm{~mW}$ and $16.5 \mathrm{~mW}$ respectively.

Overall the efficiency is at least $2 \mathrm{x}$ better than [2] and $5 \mathrm{x}$ better than [11] at lower frequency. For higher frequency [11] does not use the multipath mixer, but still the power efficiency of the current design is better.

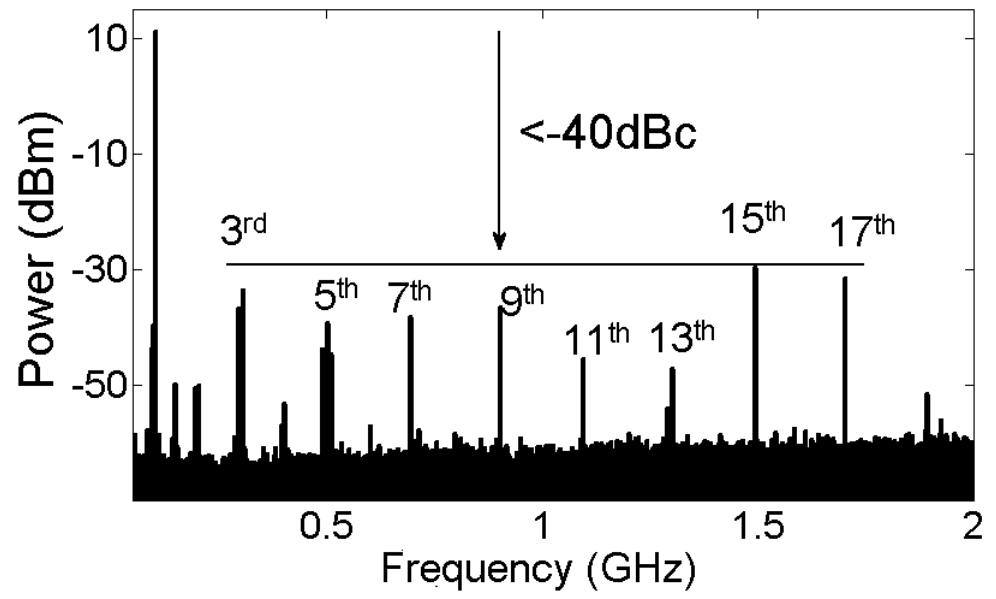

Figure 5.23 Measured wideband spectrum for $100 \mathrm{MHz} \mathrm{LO}$

Spectral purity was tested in several ways. A wideband spectrum for a $2.5 \mathrm{MHz}$ single tone upconverted by a $100 \mathrm{MHz}$ LO is shown in Figure 5.23. The harmonics are at $<-45 \mathrm{dBc}$ upto the 15 th harmonic, while the 15 th and 17 th harmonic are also $\leq-40 \mathrm{dBc}$. A 2-tone test showed an OIP3 of $+21 \mathrm{dBm}$ at $100 \mathrm{MHz}$ shown in Figure 5.24. At higher frequencies this gradually drops to $+18 \mathrm{dBm}$ mainly due to the drop in fundamental signal power. The thermal noise was measured to be $-153 \mathrm{dBc} / \mathrm{Hz}$ at $35 \mathrm{MHz}$ offset from the carrier. Below this offset the quantization noise of the 8-bit DAC and the baseband filter is dominant. The transmitter was tested with phase shifted signals generated digitally, but interestingly it is also possible to apply an analog signal centered at the sampling frequency and use the on 
chip de-interleaver to analogly produce the required polyphase signals, similar to [12]. In order to get an idea of the EVM performance, a DVB-T like OFDM signal is tested with 2048 sub carriers, in a $9.14 \mathrm{MHz}$ BW, 1705 of which actively carry data (64-QAM) and pilot tones (BPSK) upconverted to $128 \mathrm{MHz}$ shown in Figure 5.25. An EVM of $-30 \mathrm{dBc}$ (3.2\%) was achieved at $4.6 \mathrm{dBm}$ output power. A comparison with other work addressing agile broadband clean transmission is shown in Table 5.1. In comparison this design achieves better output power and efficiency, while also improving on LO Leakage and image rejection. The active area of the chip is also smaller.

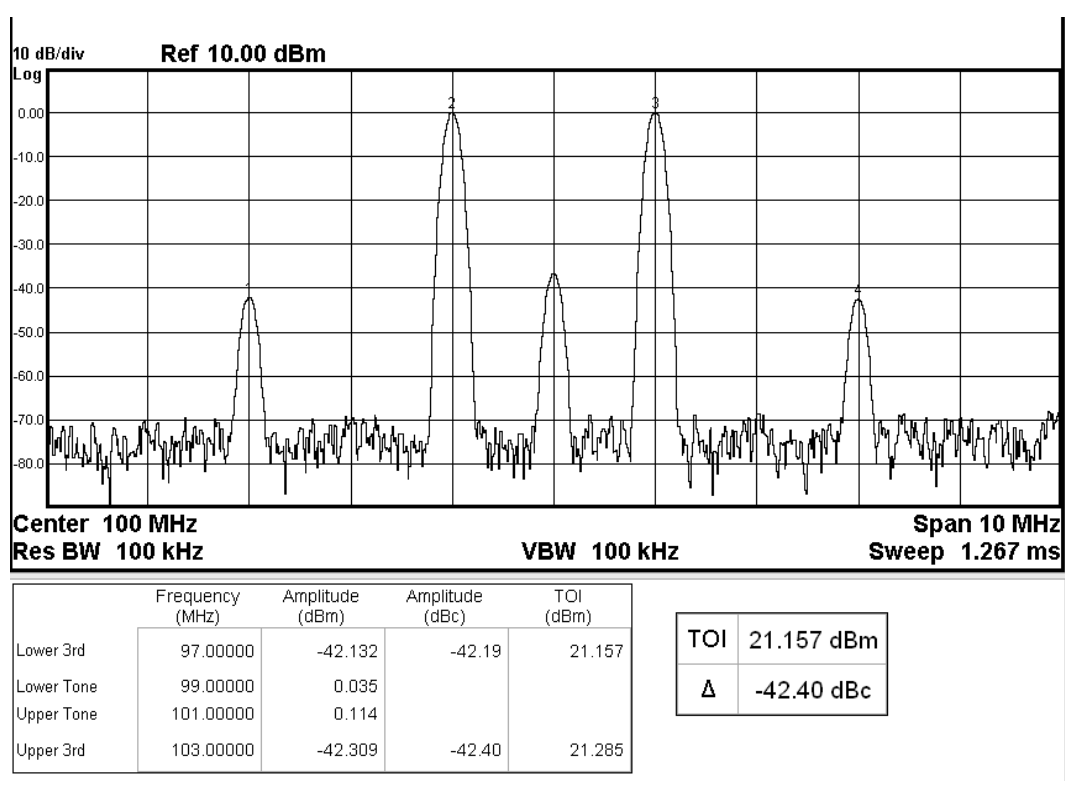

Figure 5.24 Two tone test for OIP3 

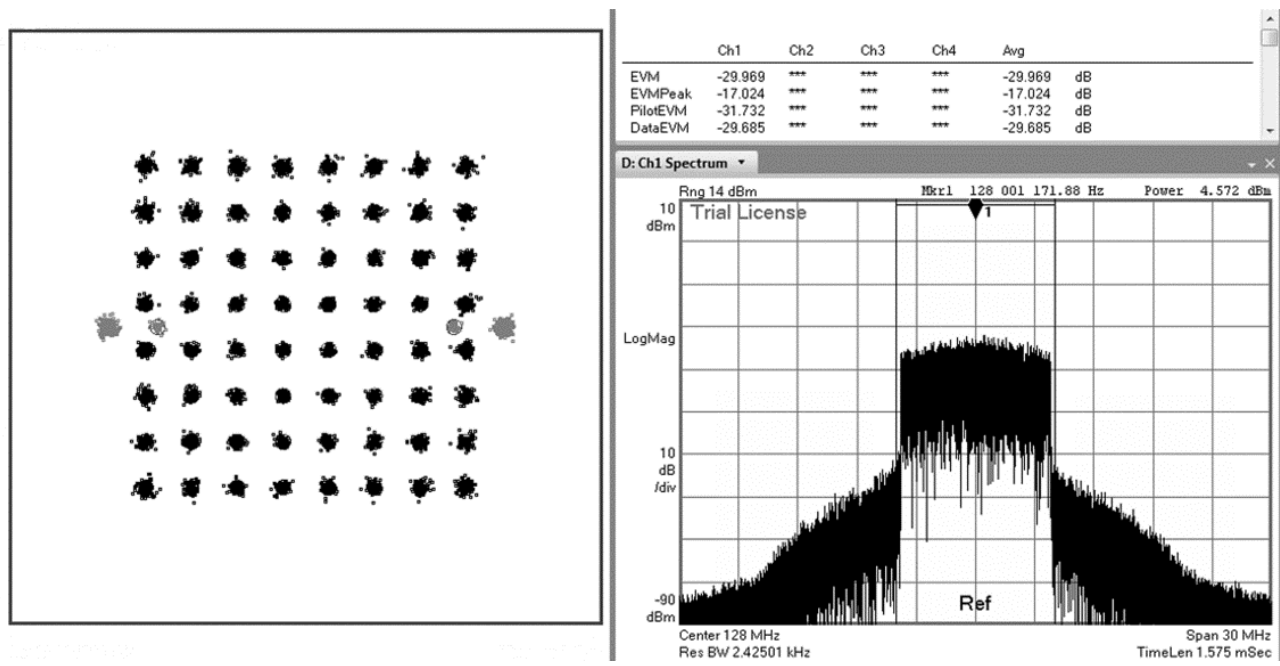

Figure 5.25 Measured Output Spectrum and Signal Constellation for a 64 QAM OFDM signal.

\subsection{Conclusion}

A frequency agile 8-path polyphase transmitter concept and circuit implementation has been presented, achieving a $<-40 \mathrm{dBc}$ clean output spectrum for all harmonics. This is the first multi-path polyphase transmitter combining the baseband multi-phase generation and RF circuit on one chip, together with a new duty-cycle control circuit which simultaneously suppresses the $7^{\text {th }}$ and $9^{\text {th }}$ LO harmonics. The simple but effective duty-cycle control circuit can produce a close to $43.65 \%$ duty-cycle with less than $1 \mathrm{~dB}$ error from the optimal point. Overall the agile power upconverter works over 3 octaves in frequency from $100 \mathrm{MHz}$ to $800 \mathrm{MHz}$. Compared with other designs with similar frequency range, it is more power efficient, has better LO leakage and image rejection. The maximum efficiency and OIP3 is better than $8 \%$ and $21 \mathrm{dBm}$ respectively, while the image strength and LO Leakage is also better than $-45 \mathrm{dBc}$. It suppresses ALL LO harmonics without any external filters. 
Table 5.1 Benchmarking to other Harmonic Rejection Transmitters.

\begin{tabular}{|c|c|c|c|c|c|}
\hline & \multicolumn{2}{|c|}{ This work } & Shrestha & \multicolumn{2}{|c|}{ Kim [11] } \\
\hline $\begin{array}{l}\text { Technology } \\
\text { Supply voltage }\end{array}$ & \multicolumn{2}{|c|}{ 160nm CMOS } & $\begin{array}{l}\text { 130nm } \\
\text { CMOS }\end{array}$ & \multicolumn{2}{|c|}{ 180nm CMOS } \\
\hline Frequency & \multicolumn{2}{|c|}{ 100-800MHz } & 30-800MHz & \multicolumn{2}{|c|}{$54-862 \mathrm{MHz}$} \\
\hline $\begin{array}{l}\text { Harmonic Rejection } \\
\text { Principle }\end{array}$ & \multicolumn{2}{|c|}{$\begin{array}{l}\text { Polyphase 8-path } \\
\text { +duty-cycle control }\end{array}$} & $\begin{array}{l}\text { Polyphase } \\
\text { 18-path } \\
+1 / 3 \text { duty- } \\
\text { cycle }\end{array}$ & \multicolumn{2}{|c|}{$\begin{array}{l}\text { Harmonic Rejection } \\
\text { Mixer } \\
\text { + active gm-C filter } \\
\quad+\text { LC notch filter }\end{array}$} \\
\hline Output $\mathbf{P} 1_{\mathrm{dB}}$ & \multicolumn{2}{|c|}{$9 \sim 10.8 \mathrm{dBm}$} & $9 \mathrm{dBm}$ & \multicolumn{2}{|c|}{$6.4 \sim 8.8 \mathrm{dBm}$} \\
\hline Power Consumption & @100MHz & @500MHz & @? & @100MHz & @500MHz \\
\hline Upconverter Mixers & $88 \mathrm{~mW}$ & $88 \mathrm{~mW}$ & $72 \mathrm{~mW}$ & $171 \mathrm{~mW}$ & $131 \mathrm{~mW}$ \\
\hline + Baseband circuits & $+30 \mathrm{~mW}$ & $+30 \mathrm{~mW}$ & NA & & \\
\hline Multiphase clock & $+11 \mathrm{~mW}$ & $+33 \mathrm{~mW}$ & +156 mW & $83 \mathrm{~mW}^{*}$ & $40 \mathrm{~mW}^{*}$ \\
\hline$P_{\text {total }}$ & $129 \mathrm{~mW}$ & $151 \mathrm{~mW}$ & $228 \mathrm{~mW}$ & $254 \mathrm{~mW}$ & $171 \mathrm{~mW}$ \\
\hline Efficiency $\left(P 1_{d B} / P_{\text {tot }}\right)$ & $8.7 \%$ & $6.7 \%$ & $<3.5 \% * *$ & $1.7 \% *$ & $4.4 \% *$ \\
\hline OIP3 & \multicolumn{2}{|c|}{$18^{\sim} 21 \mathrm{dBm}$} & NA & \multicolumn{2}{|c|}{$15.9 \sim 21.7 \mathrm{dBm}$} \\
\hline $\begin{array}{l}\text { LO Leakage \& Image } \\
\text { Strength }\end{array}$ & \multicolumn{2}{|c|}{$<-45 d B c$} & $<-35 d B c$ & \multicolumn{2}{|c|}{$<-41 d B c$} \\
\hline Strongest Harmonic & \multicolumn{2}{|c|}{$<-40 \mathrm{dBc}$} & $<-31 d B c$ & \multicolumn{2}{|c|}{$<-42 \mathrm{dBc}$} \\
\hline Noise & \multicolumn{2}{|c|}{$-153 \mathrm{dBc} / \mathrm{Hz} @ 35 \mathrm{MHz}$} & NA & \multicolumn{2}{|c|}{$-122 \mathrm{dBc} / \mathrm{Hz} @ 1 \mathrm{MHz} *$} \\
\hline $\begin{array}{l}\text { Area } \\
\text { Transmitter } \\
\text { Baseband Filter }\end{array}$ & & $m^{2}$ & $\begin{array}{c}0.14 \mathrm{~mm}^{2} \\
\mathrm{NA}\end{array}$ & $\begin{array}{r}2.2 \mathrm{~m} \\
2 \mathrm{~m}\end{array}$ & **** \\
\hline
\end{tabular}

*includes $\mathrm{VCO} / \mathrm{PLL} * *$ No baseband circuits $* * *$ Estimated from chip micrograph 


\section{References}

[1] S. Subhan, E. A. M. Klumperink, A. Ghaffari, G. J. M. Wienk, and B. Nauta, "A 100-800 MHz 8-Path Polyphase Transmitter With Mixer Duty-Cycle Control Achieving < $-40 \mathrm{dBc}$ for ALL Harmonics," Solid-State Circuits, IEEE Journal of, vol. 49, pp. 595-607, Mar. 2014.

[2] R. Shrestha, E. A. M. Klumperink, E. Mensink, G. J. M. Wienk, and B. Nauta, "A Polyphase Multipath Technique for Software-Defined Radio Transmitters," Solid-State Circuits, IEEE Journal of, vol. 41, pp. 2681-2692, 2006.

[3] S. M. Louwsma, A. J. L. van Tuijl, M. Vertregt, and B. Nauta, "A 1.35 GS/s, 10b, 175mW Time-Interleaved AD Converter in 0.13um CMOS," SolidState Circuits, IEEE Journal of, vol. 43, pp. 778-786, Apr. 2008.

[4] S. Balasubramanian and W. Khalil, "Direct digital-to-RF digital-to-analogue converter using image replica and nonlinearity cancelling architecture," Electronics Letters, vol. 46, pp. 1030-1032, 2010.

[5] S. Subhan, E. A. M. Klumperink, and B. Nauta, "Towards suppression of all harmonics in a polyphase multipath transmitter," in Circuits and Systems (ISCAS), 2011 IEEE International Symposium on, 2011, pp. 2185-2188.

[6] X. Gao, B. Nauta, and E. A. M. Klumperink, "Advantages of Shift Registers Over DLLs for Flexible Low Jitter Multiphase Clock Generation," Circuits and Systems II: Express Briefs, IEEE Transactions on, vol. 55, pp. 244-248, 2008.

[7] R. Dutta, T. K. Bhattacharyya, X. Gao, and E. Klumperink, "Optimized Stage Ratio of Tapered CMOS Inverters for Minimum Power and Mismatch Jitter Product," in VLSI Design, 2010. VLSID '10. 23rd International Conference on, 2010, pp. 152-157.

[8] E. Klumperink, R. Dutta, R. Zhiyu, B. Nauta, and X. Gao, "Jitter-Power minimization of digital frequency synthesis architectures," in Circuits and Systems (ISCAS), 2011 IEEE International Symposium on, 2011, pp. 165168.

[9] E. A. M. Klumperink, S. M. Louwsma, G. J. M. Wienk, and B. Nauta, "A CMOS switched transconductor mixer," Solid-State Circuits, IEEE Journal of, vol. 39, pp. 1231-1240, 2004.

[10] E. Mensink, E. A. M. Klumperink, and B. Nauta, "Distortion cancellation via polyphase multipath circuits," in Circuits and Systems, 2004. ISCAS '04. Proceedings of the 2004 International Symposium on, 2004, pp. I-1098101 Vol.1. 
[11] K. Jongsik, L. Seung Jun, K. Seungsoo, H. Jong Ok, E. Yun Seong, and S. Hyunchol, "A 54-862-MHz CMOS Transceiver for TV-Band White-Space Device Applications," Microwave Theory and Techniques, IEEE Transactions on, vol. 59, pp. 966-977, 2011.

[12] A. Ghaffari, E. A. M. Klumperink, M. C. M. Soer, and B. Nauta, "Tunable High-Q N-Path Band-Pass Filters: Modeling and Verification," Solid-State Circuits, IEEE Journal of, vol. 46, pp. 998-1010, 2011. 


\section{CHAPTER SIX}

\section{Conclusions}

\subsection{Summary and Conclusions}

This thesis aims at the design of a transmitter for cognitive radio, which is built on the Software Defined Radio concept, where one radio frequency frontend has the ability to meet the functionality of various standards. The cognitive radio front end has the added functionality of first sensing and then transmitting in the unutilized frequency spectrum, i.e. exploit dynamic spectrum access to use the frequency spectrum more efficiently. The Cognitive Radio transmitter would need to be flexible to be able to transmit where ever there is free spectrum available. It should also have some mechanism to suppress the out of band harmonics and distortion products which result due to the time-variant and non-linear upconversion process. These out of band harmonics may interfere with other users of the frequency spectrum. The Cognitive Radio transmitter should avoid the use of dedicated RF band pass filters for each different RF band it utilizes, since that would make the design bulky and costly.

The goal of this work is to design a flexible transmitter which does not require dedicated $\mathrm{RF}$ filters to suppress harmonics and distortion produced during upconversion, while having competitive power efficiency. In chapter 2 a review of some of the multimode transmitter architectures in recent literature is presented. These architectures require dedicated RF filters to suppress harmonics. The harmonic suppression techniques including the polyphase multipath technique (a modification of which is proposed in chapter 3) is also discussed. These harmonic suppression techniques relax the RF filtering requirements and are suitable for cognitive radio transmitters. The out of band emission requirements on the cognitive radio transmitter are also discussed.

In chapter 3 a technique was proposed which utilizes an 8-path transmitter, while utilizing a duty cycle of the $\mathrm{LO}$ of $43.65 \%$, which in combination with $1^{\text {st }}$ order tunable RF filter was able to suppress all the harmonics to below $40 \mathrm{dBc}$. This can be improved further if a high $\mathrm{Q}$ tunable RLC network is used at the output but at the cost of reduction in tuning range. Using only 8-paths would reduce the power consumption of the LO generation compared to previous designs, and also reduce the complexity of the baseband circuits. Analysis 
indicates that the power consumption of the upconverter using $43.65 \%$ duty cycle is also improved as compared to $50 \%$ duty cycle, while the un-cancelled harmonics almost fully add up in phase.

In chapter 4 it is shown that it is possible to generate the multiphase baseband signals required for an 8-path upconversion by using simple vector addition, using a simplified vector modulator type of architecture. The multipath polyphase transmitter design can in principle cancel harmonics responses in the DAC output resulting from quantization errors. However, since inter-modulation terms remain un-cancelled so its usefulness is limited. In order to keep the SFDR due to limited DAC resolution better than $50 \mathrm{~dB}$ at least a 7 bit $\mathrm{DAC}$ resolution is required.

Also in Chapter 4 it is shown that it is possible to cancel the first dominant DAC image by using polyphase DAC architecture. The dominant non-cancelled image is then at $2 \times \mathrm{fs}$, which occurs due to its almost in-phase addition in all the paths. It is possible to implement either a single DAC on chip, using a single voltage reference and multiple switching networks, or a single DAC and de-interleaving switches and hold functions to make the 8 analog baseband signals. For testing flexibility and evaluation purposes one external DAC and on chip de interleaving was chosen. Digital interpolation filters can relax the reconstruction filtering requirement to a second order filter with limited power overhead.

In Chapter 5 a demonstrator chip fabricated in a 160nm CMOS process is presented. It is the first multi-path polyphase transmitter combining the baseband multi-phase generation and RF circuit on one chip. A new duty-cycle control circuit was designed which simultaneously suppresses the $7^{\text {th }}$ and $9^{\text {th }}$ LO harmonics. The simple but effective dutycycle control circuit can produce a close to $43.65 \%$ duty-cycle with less than $1 \mathrm{~dB}$ error from the optimal point. The measurements show that the frequency agile 8-path polyphase transmitter can achieve a $<-40 \mathrm{dBc}$ clean output spectrum for all harmonics. Overall the agile power up-converter works over 3 octaves in frequency from $100 \mathrm{MHz}$ to $800 \mathrm{MHz}$. Compared to other designs with similar frequency range, it is more power efficient, has better LO leakage and image rejection. It suppresses ALL LO harmonics without any external filters.

\subsection{Original Contributions}

- The discovery of an optimum for the duty cycle ratio of the local oscillator to simultaneously suppress the $7^{\text {th }}$ and $9^{\text {th }}$ harmonics of the LO. 
- The design of a flexible $100-800 \mathrm{MHz}$ transmitter that suppresses ALL the harmonics to below $-40 \mathrm{dBc}$ combining three techniques: 1) an 8-path polyphase upconverter; 2) the optimum duty cycle ratio; 3 ) a simple $1^{\text {st }}$ order passive filter.

- The design of a duty cycle control circuit that is able to keep the duty cycle variation $<0.1 \%$, while covering a $100-800 \mathrm{MHz}$ multi-octave frequency range.

- The use of one DAC to generate all the baseband phases required for the multipath up-conversion, with reduced mismatch errors between paths.

- The implementation of the complete baseband to RF polyphase 8-path upconversion in $160 \mathrm{~nm}$ CMOS.

- The digital design and synthesis to generate the 8-phase polyphase baseband signals.

- The analysis of the DAC image cancellation properties of the polyphase multipath upconversion.

\subsection{Recommendations for Future Work}

- Since the polyphase multipath technique does not cancel the inter-modulation products, it could be useful to incorporate some form of pre-distortion which could linearize the frontend further and improve the inter-modulation performance.

- With a spectrum analyzer on board it should be possible to measure the effect of the phase or amplitude mismatch. To overcome the phase or amplitude mismatch, it could be possible to detect the mismatch and try to compensate in the digital domain or by fine-tuning LO-phases.

- In order to make the polyphase transmitter even more flexible, so that it can cater to a variety of baseband signals, it is recommended to experiment with higher baseband DAC-speeds or even a direct digital to RF (or an RF-DAC) approach. This would eliminate the baseband filters and make the design more flexibly programmable, but could pose issues related to unwanted spurious emissions (still requiring filtering) and the power consumption of the DAC and high-speed digital 
design. Especially in new CMOS technologies this option seems increasingly appealing. 


\section{APPENDIX A}

MATLAB code for section 4.2 .2 (where $\omega_{1}$ and $\omega_{2}$ are the radial frequency of the two input tones)

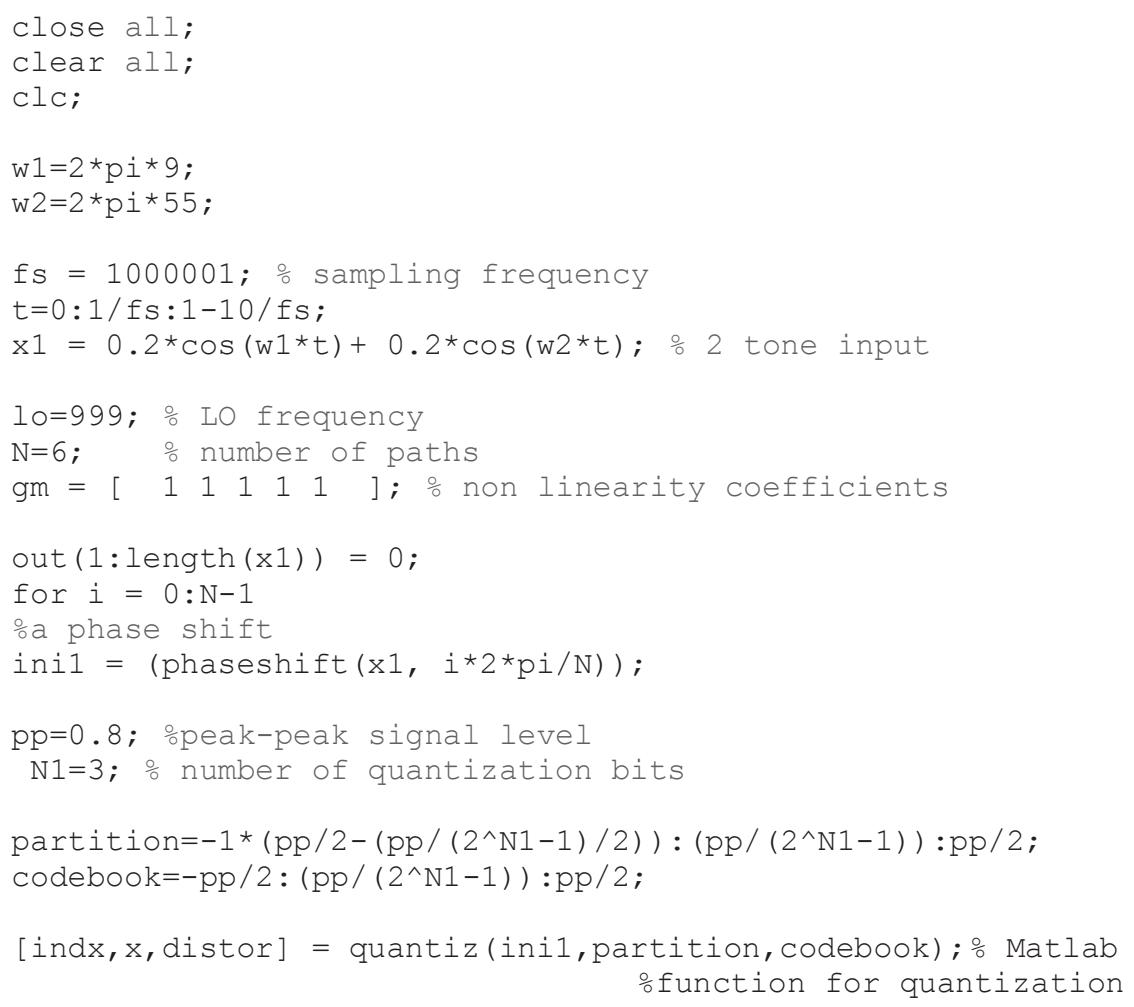

figure;

plot (out1); axis([0 $8000-60$ 0]); ylabel('Magnitude

$(\mathrm{dB})$ ') ; xlabel ('Frequency'); title('After Addition of ALL Paths'); 


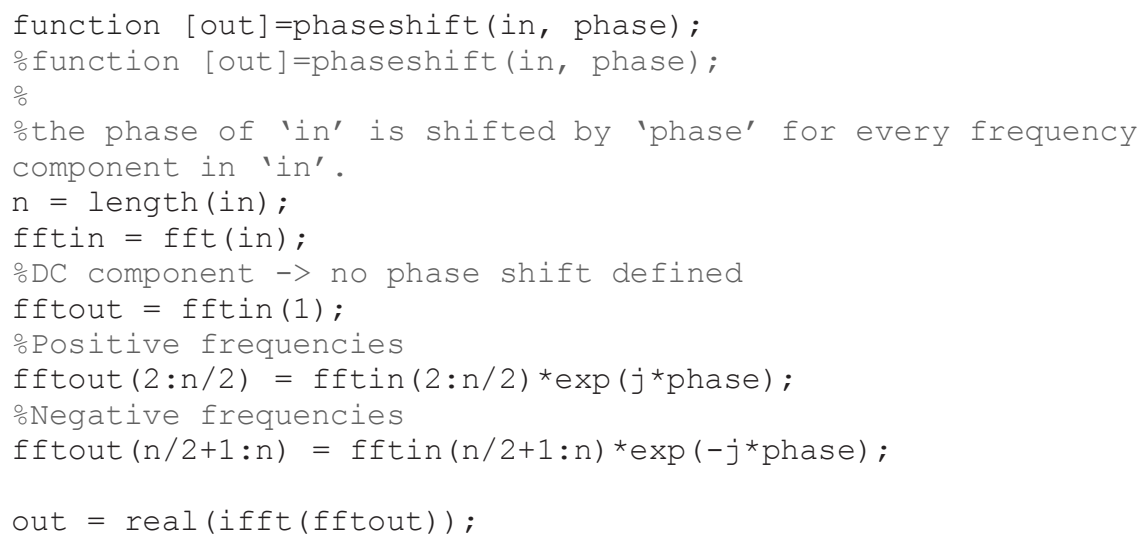




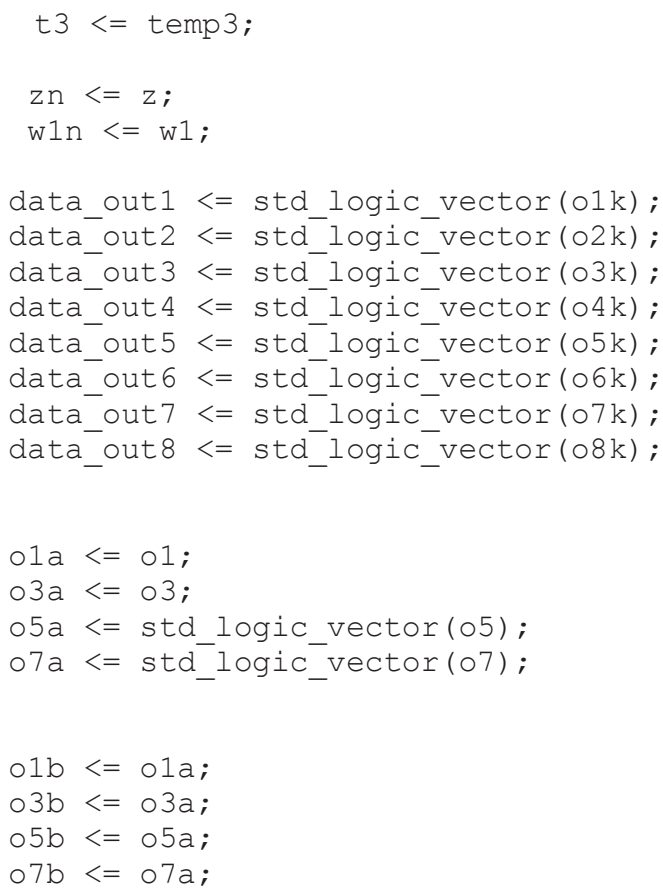

$\mathrm{z}<=$ unsigned(t1 (2*word length-5 downto word length-10)) tunsigned(t2 (2*word_length-5 downto word_length-10)) ; w1 <= unsigned(t2 ( $2{ }^{*}$ word_length-5 downto word_length-10)) tunsigned(t3 (2*word_length-5 downto word_length-10)) ;

end if;

end process seq;

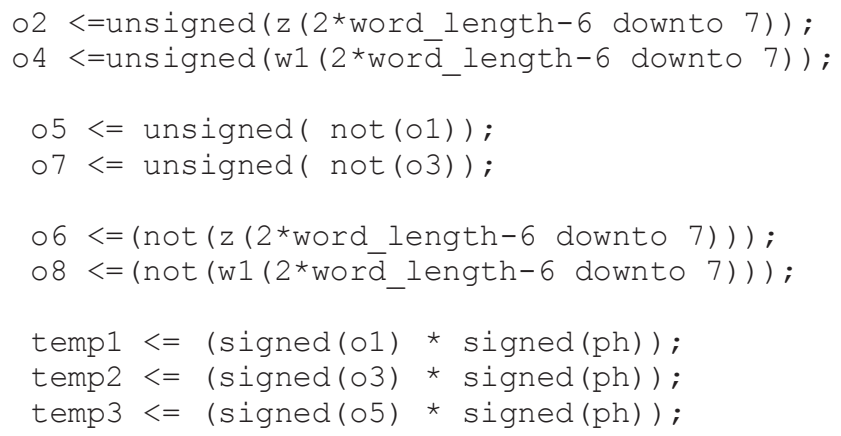

$\circ 1 \mathrm{k}<=$ unsigned (o1b); 


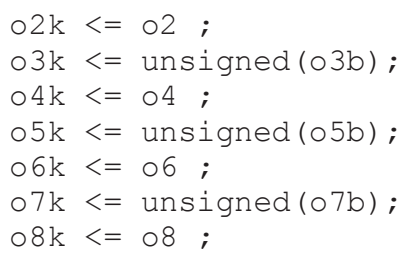

end multiply; 


\section{APPENDIX C}

\section{VHDL code for Interpolation Filter Architecture}

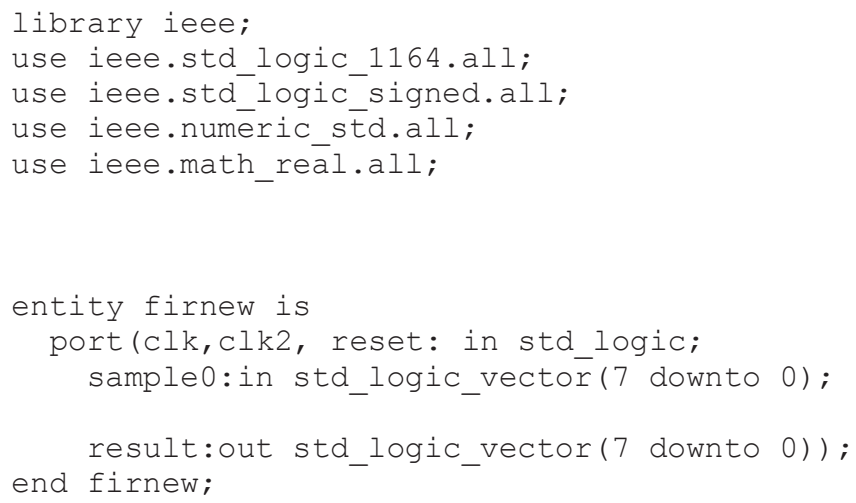




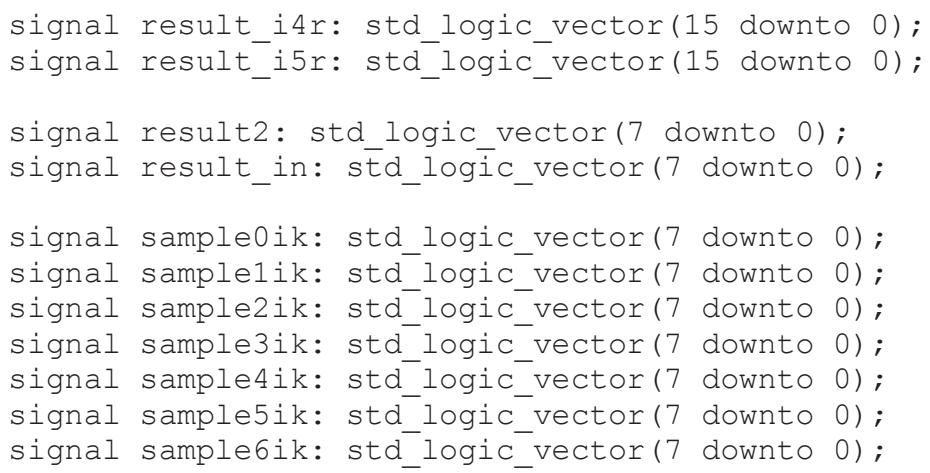

begin

seq2: process (clk, reset)

begin

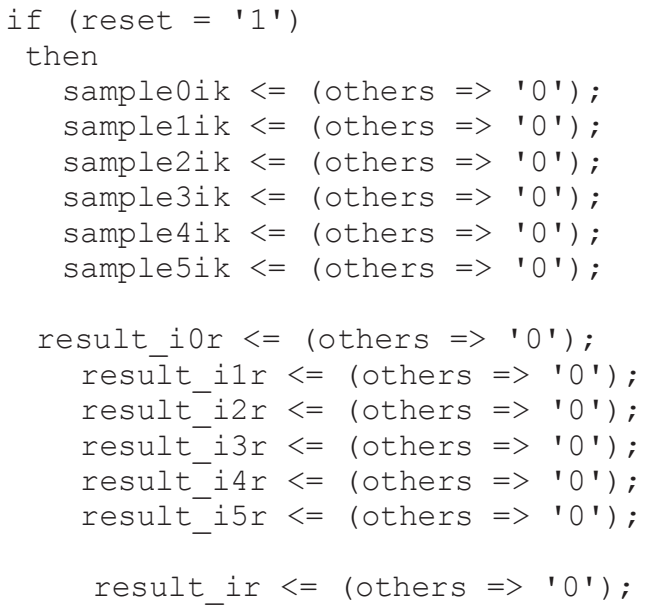




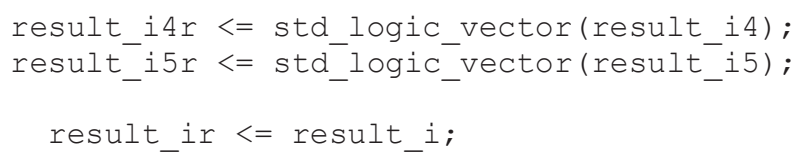

end if;

end process seq2;

seq:process (clk2, reset)

begin

if $($ reset $=' 1$ ')

then

sample6ik $<=$ (others $=>$ 'O') $^{\prime}$;

result $<=$ (others $=>$ '0');

elsif rising edge(clk2)

then

sample6ik <= sample4ik;

result $<=$ result2;

end if;

end process seq;

seq3 3 process (clk)

begin

if $(\mathrm{clk}=$ ' 1 ')

then

result $2<=$ result_in;

else

end if;

result2 <= sample6ik;

end process seq3;

result i0<= unsigned(unsigned( sample0ik)*unsigned(c0)); resultil <= unsigned (unsigned (samplelik)*unsigned (c1));

result_i2 <= unsigned(unsigned(sample2ik)*unsigned(c2));

result_i3<= unsigned (unsigned (sample3ik)*unsigned (c3));

result_i4 <= unsigned(unsigned(sample4ik)*unsigned(c4));

result_i5<= unsigned(unsigned(sample5ik)*unsigned(c5));

result_i $<=$ result_i0r-result_ilr+result_i2r+result_i3rresult_i4r+result_i5r;

result_in $<=$ result_ir(14 downto 7);

end beh; 


\section{Acknowledgements}

This thesis has been made possible with the direct and indirect support and help of many individuals and organizations. I would like to thank them here for their cooperation.

First of all I would thank Bram Nauta, who accepted my request to join his IC Design group to pursue research. It provided me a great learning opportunity and allowed me to experience the world of circuits and systems. Although he always had a busy schedule but during our meetings, he would often come up with interesting and critical comments which allowed me to think of things in a different light. His ambitious approach towards research was inspiring and allowed me to see what it takes to be one of the best research groups in the field of IC Design. My stay at the group coincided with a very productive period in which many of the publications of the group were accepted to the top most conference in the field of Integrated Circuits. I was fortunate enough to observe and learn the work ethic required to achieve this success. It was instructive to see the chip making process from the informal chip talks on Friday afternoons, the progress meetings, to the multiple design reviews, the layout reviews and the attention to detail required in achieving a working integrated circuit. I am thankful to Bram and the entire ICD team for allowing me to experience this.

My daily supervisor was Eric Klumperink. I thank him for his guidance and patience at every stage of my project. Many a times his ideas and encouragement got me through difficult times. His work rate was inspiring to say the least. His ability to make a rather less coherent paper into a very readable one has definitely helped me a lot. His guidance has allowed me to read and listen to scientific literature much more critically. In short he was as close to an ideal mentor as there could be. If I ever have an opportunity to guide others, I would surely have a great example to emulate. I would especially like to thank Eric and Bram for arranging the funding for my last year of stay at the group.

My stay at the ICD Design group was initially made possible due to a scholarship from the Higher Education commission (HEC) of the Government of Pakistan. I would like to thank its leadership at the time of Prof. Atta-ur-Rehman and Dr. Sohail Naqvi and their team for their ambitious plan of creating a research environment in the country. The scholarship was also administered by the Dutch Higher Education Organization NUFFIC. I would like to thank Mr. Beer Schroder and Mrs. Loes Minkman for conducting the initial interviews and the selection process in Islamabad. Also would like to thank the staff at NUFFIC for their kind cooperation throughout my stay in Netherlands. I would like to thank the Dutch Technology foundation (STW), who funded the final year of my stay at the ICD group and NXP for allowing the chip fabrication. I would like to thank Raf Roovers, Hans 
Brekelmans, and Jan van Sinderen at NXP for their support. I would like to thank Robert Rutten at NXP for useful discussions on digital design. Especial thanks to Gerard van der Weide at NXP for help in the tape-out process.

I would like to thank Gerard Wienk and Henk de Vries at the ICD group. Gerard for helping with my layout and the PCB and the CADD related issues. Henk de Vries for setting up a convenient measurement schedule, and providing the necessary measurement equipment. Their support was critical in achieving the tape-out and the measurements just in time.

I would like to thank Gerdien for helping with many administrative issues. Her kindness was a calming influence in the sea of stress sometimes. I would also like to thank her for organizing the numerous uitje (excursions) which were always enjoyable. Also would like to thank Satie for her help during her time in ICD. I would also like to thank all the members of the SC group and especially Annemiek for her help and the numerous coffee+ and making the overall atmosphere cheerful.

I would like to thank Jaap Haartsen and Andre Kokkler for being part of the progress meetings in the initial part of my project and then later on as part of the ADREM progress and user committee meetings. I would also like to thank the other members of the user committee Jan-Wim Eikenbroek, Jan Prummel, Jan van Sinderen, Kave Kianush and Wouter Segeth for their useful comments and discussions.

I would like to thank the teachers for the courses I took during my first year including Sabih Gerez, Ronan van der Zee, Hans Kerkhoff, Eric Klumperink, Marcel Pelgrom, Ed van Tuijl and Jaap Haartsen. I learned many new things from those courses.

I would like to thank my ICD colleagues. Initially one of my office mates was Paul.G. Most things about the digital tools and many tips and tricks about Spectre simulations I learned from him. Also our many discussions late into the evenings about various things were always interesting and enjoyable. I will always cherish those memories. Amir, Dlovan, Mark and Zhiyu were part of the ADREM project meetings, I thank them for the discussions and comments during those progress meetings.

Also I would like to thank Amir for his friendship and whose office I would often pop into for any technical or non-technical discussion. His suggestions were always useful and helpful. Dlovan who was also my office mate, I want to thank him for his friendship and his willingness to offer help or advice whenever he could. I thank Mark for his help with my IC measurements (those automated scripts definitely made life so much easier) and also many useful discussions. I also thank Dirk-Jan for the EVM measurements on my IC. 
I would also like to thank Stephan, Egbert, Rameswor, Niels, Michiel, Kasra, Milad, Xiang, Wei, Harish, Ramen, Remko, Jasper, Erik, Johan, David, Fawzi, Fabian, Daniel, Eisse, Paul.V, Bram.V, Shadi and Haifeng for useful discussions and a pleasant time spent in their company. I would like to thank Robert Brookhuis; working with him during the SOC lab and then the project was fun and rewarding. I also thank Pepijn and Boris for allowing me to supervise their final Bachelors project, which was also interesting for me.

I would like to thank my Pakistani fiends in Enschede for the nice time we spent together. Imran was my first contact in Enschede and he and his family always provided such a warm environment at their home, which I would remember for a long time. An especial thankyou to Hammad, Rauf, Kazmi, Saifullah, Sohail, Adeel, Tariq, Asad, Khurrem, Rahim, Ahmad.D, Farrukh, Mehdi for their friendship and support, and their families for all the BBQs and the get-togethers which I always enjoyed. My housemates Kazmi, Rauf, Muddassir, thankyou for the nice dinners and discussions. I will always have nice memories of the time spent together.

Also would like to thank Waqar, Akram, Nasir, Fawad, Fawad.K, Asif, Sajid, Hammad.F, Saba, Amna, Zaghum, Mohsin and Mohsin, Khurshid, Amir, Shuja, Taimoor, Irfan.A, Irfan.Z, Ghani, Faizan, Yawar, Salman, and Tauseef. I would like to thank the board members over the years of the Pakistan Students Association at the UT, for organizing events which were always enjoyable and fun.

At the end I would like to thank my family. My parents have been there for me always. They have always offered their unconditional love and support. My gratitude towards them cannot be expressed in words. I would like to thank my wife Ammarah, for her love and unflinching support in completing this thesis. Thank you for your understanding, especially for the times we could not spend together. Last but by no means the least, I thank my son Mustafa for always bringing a smile to my face. Life has been so much more joyful since you came into this world.

Thank You All!

June, 2014 


\section{List of Publications}

[1] S. Subhan, E. A. M. Klumperink, B. Nauta, "D/A Resolution Impact on a Polyphase Multipath Transmitter", Proceedings of the $19^{\text {th }}$ Annual Workshop on Circuits, 27 Nov 2008, Veldhoven, The Netherlands.

[2] M. S. Oude Alink, D. H. Mahrof, A. Ghaffari, S. Subhan, E. A. M. Klumperink, A. B. J. Kokkeler, G. J. M. Smit, and B. Nauta, "AD-REM radio project overview," in 15th Annual CTIT Symposium - Reinventing ICT, Enschede, The Netherlands, 16 Jun. 2009.

[3] M. S. Oude Alink, D. H. Mahrof, A. Ghaffari, S. Subhan, E. A. M. Klumperink, A. B. J. Kokkeler, G. J. M. Smit, and B. Nauta, "Cognitive radio - a revolution in wireless communications," in STW Jaarcongres, Nieuwegein, The Netherlands, 7 Oct. 2010.

[4] S. Subhan, E. A. M. Klumperink, B. Nauta, "Cognitve Radio Transmitter with a clean Output Spectrum", in $21^{\text {th }}$ Annual Workshop on Circuits, Systems and Signal Processing (ICT.Open), Veldhoven, The Netherlands, 14-15 Nov. 2011.

[5] S. Subhan, E. A. M. Klumperink, B. Nauta, "Towards Suppression of All Harmonics in a Polyphase Multipath Transmitter", International Symposium on Circuits and Systems (ISCAS), 15-18 May 2011, Rio de Janeiro, Brazil.

[6] S. Subhan, E. A. M. Klumperink, A. Ghaffari, G. J. M. Wienk, and B. Nauta, "A 100-800 MHz 8-Path Polyphase Transmitter With Mixer Duty-Cycle Control Achieving < $-40 \mathrm{dBc}$ for ALL Harmonics," Solid-State Circuits, IEEE Journal of, vol. 49, pp. 595-607, Mar. 2014. 


\section{About the Author}

Saqib Subhan was born in Lahore, Pakistan, in 1981. He received the B.S. degree in electronic engineering from the GIK Institute, Pakistan, in 2003 and the M.S. degree in Systems Engineering from the Pakistan Institute of Engineering and Applied Sciences in 2005. In 2007 he was awarded the overseas scholarship of the Higher Education Commission of Pakistan to pursue graduate studies. From 20072012 he was with the IC-Design group of the University of Twente, Enschede, The Netherlands, working towards the $\mathrm{PhD}$ degree on the subject of Cognitive Radio Transmitters in CMOS. His research interests include analog and mixed-signal RFIC and digital design. 